2D/3D REGISTRATION ALGORITHM FOR LUNG BRACHYTHERAPY 


\title{
2D/3D REGISTRATION ALGORITHM FOR LUNG BRACHYTHERAPY
}

by

\author{
PAVEL ZVONAREV, M.Sc., B.Sc. \\ A Thesis \\ Submitted to the School of Graduate Studies \\ In Partial Fulfillment of the Requirements \\ for the Degree \\ Doctor of Philosophy
}

McMaster University

(c) Copyright by Pavel Zvonarev, June 2013

Doctor of Philosophy (2013)

McMaster University

(Medical Physics)

Hamilton, Ontario 
TITLE: 2D/3D Registration Algorithm for Lung Brachytherapy

AUTHOR: Pavel, Zvonarev, M.Sc. (Yaroslavl State University, Russia), B.Sc. (Yaroslavl State University, Russia)

SUPERVISOR: Professor Thomas J. Farrell

NUMBER OF PAGES: xiii, 100. 
To my parents 


\section{Acknowledgements}

I am very thankful to four individuals who guided and mentored me throughout the research of this thesis. First and foremost, I would like to thank my supervisor Dr. Tom Farrell for his advice, patience and encouragement during the course of the research.

I extend my gratitude toward Dr. Robert Hunter, Dr. Marcin Wierzbicki and Dr. Joseph Hayward for their numerous valuable suggestions, supervision and support of my work. I want to thank them all for taking the time to serve on my committee meetings. Their vast experience and deepest knowledge of the industry has helped greatly to progress with my research and get the results.

Among many people who helped me during the course of the years, I would like to especially thank Dr. Ranjan Sur for helping to collect much needed for my research patient data. I would like to thank Amelia Timotin for her assistance with patient and phantom data collection. I would like to thank Doug Wyman and Troy Farncombe for taking time to serve on my committee meetings at early stages of this research.

A big part of the great and enjoyable experience at Juravinski Cancer Centre was the medical physics group. I would like to thank Janos, Diana and Marija for their support, reading and editing my manuscript.

I want to thank my wife who supported me all the way during the research. 


\section{Abstract}

The typical High Dose Rate (HDR) lung brachytherapy procedure involves inserting treatment catheters into the bronchi next to the tumour location using a bronchoscope. The anterior-posterior and lateral fluoroscopy images are acquired in order to localize the catheters prior to treatment. Although, these images enable accurate reconstruction of the catheter location, they do not allow for the visualization of the tumour or organs-at-risk due to poor soft tissue contrast. Although CT images offer an improved soft tissue contrast, moving the patient with catheters in place prior to each treatment is impractical.

An alternative option is to use prior diagnostic or external beam radiation treatment planning CT images. These images cannot be used for treatment planning directly because of the variation in patient positioning between the CT and orthogonal images acquisition. In order to account for positioning differences, a 2D/3D registration algorithm that registers the orthogonal images with a previously acquired CT data was developed. The algorithm utilizes a rigid registration model based on a pixel/voxel intensity matching approach. A similarity measure combining normalized mutual information (NMI), image gradient, and intensity difference was developed. Evaluation of the algorithm was performed using tissue equivalent phantoms, and, in the clinical setting using data from six patients. The mean registration error was $2.1 \mathrm{~mm}$ and $3.2 \mathrm{~mm}$ for phantoms and patients respectively.

External objects such as the treatment table and ECG leads are often in CT images, affecting the above mentioned 2D/3D registration. To address this, an algorithm for automatic removal of external objects from CT images was developed. This was applied to automatic contouring and removal of the fiducial markers in CT images used for external beam radiation therapy treatment planning for breast cancer. The algorithm was further modified to compute the girth of patients as part of a diagnostic radiology clinical trial. 


\section{CONTENTS}

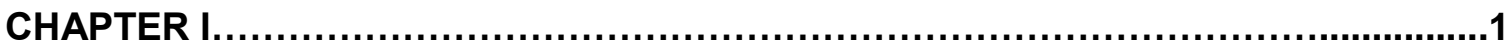

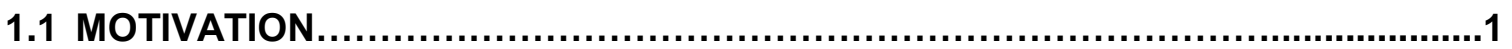

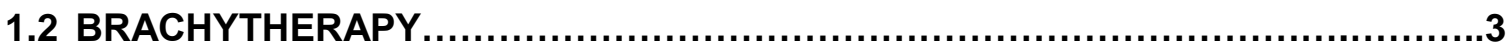

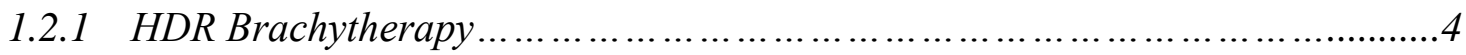

1.2.2 HDR Brachytherapy Treatment Planning ..........................................

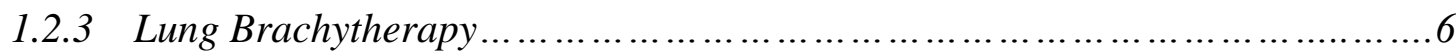

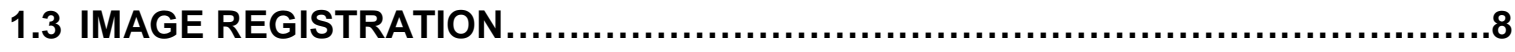

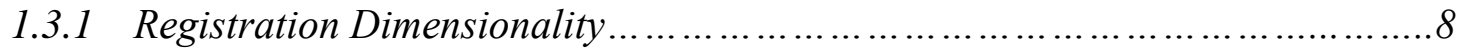

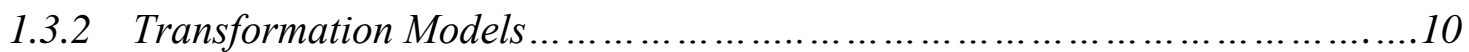

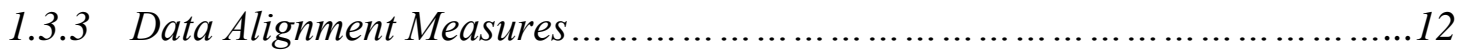

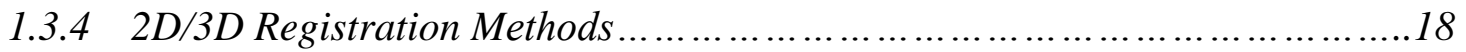

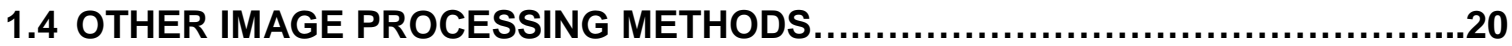

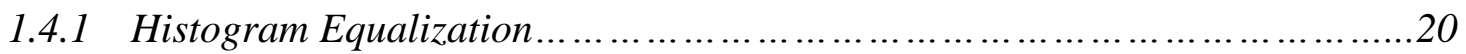

1.4.2 Mathematical Morphology Methods.............................................22

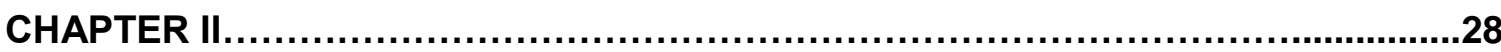

PAPER I: 2D/3D registration algorithm for lung brachytherapy...........................28

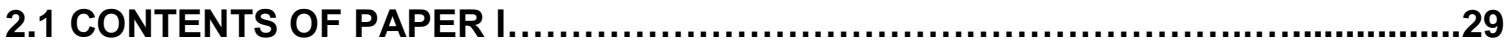

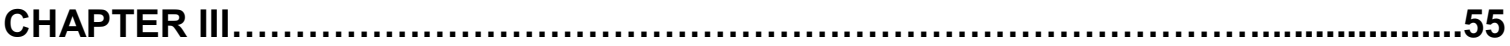

PAPER II: Clinical evaluation of a 2D/3D registration algorithm for lung

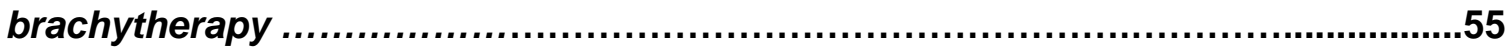

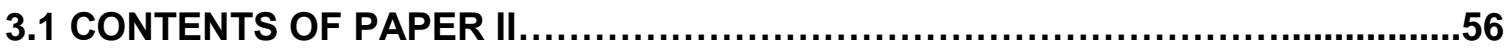

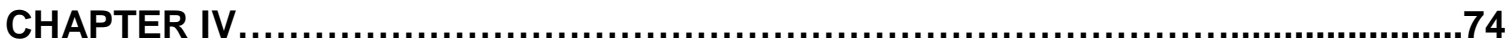

PAPER III: Automated algorithm for identification and removal of external objects from patient CT scans with applications in radiation therapy and radiology 


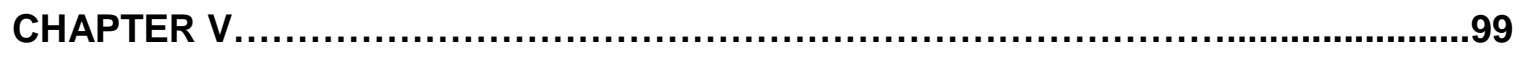

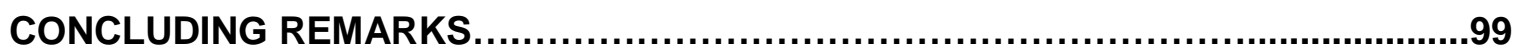

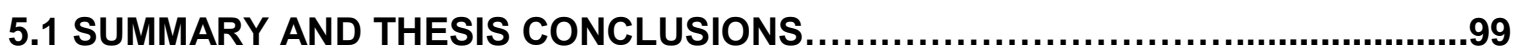

5.2 STUDY LIMITATIONS AND FUTURE DIRECTIONS ................................102

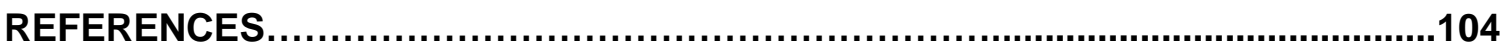




\section{List of Figures}

\section{Chapter I}

Figure 1 (a) Varian HDR unit (VariSource); (b) Rotating turret with channels (Khan et al., 2009).

Figure 2 Typical HDR brachytherapy treatment setup showing patient with inserted catheters, carm, and radiation source afterloader.

Figure 3 (a) Schematic of ray casting method; (b) Bi-planar geometry $\ldots \ldots \ldots \ldots \ldots \ldots \ldots \ldots \ldots . \ldots$

Figure 4 Typical pair of x-ray images and lung brachytherapy treatment plan: (a) Lateral x-ray image with catheters digitized catheters; (b) PA x-ray image with digitized catheters; (c) 3D rendering of the PTV and reconstructed catheters; (d) Transverse slice dose distribution; (e) Coronal slice dose distribution; (f) Sagittal slice dose distribution

Figure 5 Joint histogram plot of the brain MRI image with itself for different rotations: (a) no rotation; (b) $2^{\circ}$; (c) $10^{\circ}$ (Pluim et al., 2003)...

Figure 6 Example of the image processed by histogram equalization filter: (a) Original image; (b) Intensity histogram and cumulative distribution function of original image; (c) Processed image; (d) Intensity histogram and cumulative distribution function of the processed image (Wang, 2012)......

Figure 7 Standard structuring elements (a) 4-connected set; (b) 8-connected set. (Young et al., 1998).

Figure 8 Example of an image processed by MM filters: (a) Original image; (b) Threshold filter (50\%); (c) Dilation; (d) Erosion; (e) Opening; (f) Closing; (g) Gradient; (h)

\section{Chapter II}

Figure 1 (a) Simple body phantom; (b) Anthropomorphic thorax phantom. .38

Figure 2 (a) Normalized registration function and its components vs. translational shift in the $x$ direction; (b) normalized registration function versus rotational shift about the $\mathrm{x}$-axis.

Figure 3 (a) Registration accuracy versus $T R E_{\text {init }}$; (b) Computational time versus $T R E_{\text {init }}$ 
Figure 4 Algorithm failure rate versus $T R E_{\text {init }}$ for combined similarity metric and its

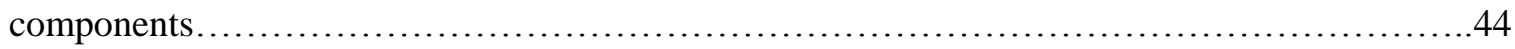

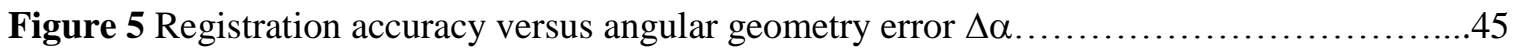

Figure 6 Example of successful registration for the anthropomorphic phantom: (a) PA X-ray image with catheters and wires; (b) Corresponding PA DRR generated from CT volume with no catheters; (c) Lateral x-ray image with catheters and wires; (d) Corresponding Lateral DRR

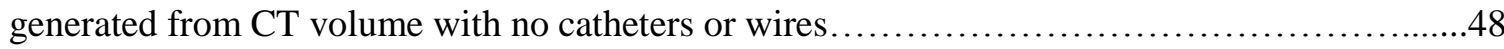

Figure 7 Diagnostic CT image slices of a lung patient showing the true (red) and registrationderived (green) catheter positions. (a) Coronal plane; (b) Transverse plane; (c) Sagittal plane; (d) $3 \mathrm{D}$ rendering....

Table 1 Summary of the algorithm's numerical evaluation results for the data that met the

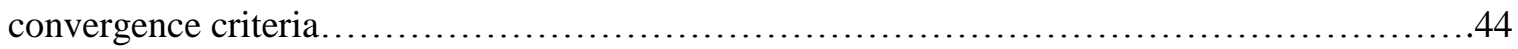

Table 2 Registration accuracy evaluation for the anthropomorphic phantom

Table 3 Algorithm failure rate for combined similarity metric, its components and their combinations.

Table 4 Digitization error. .49

Table 5 Algorithm precision analysis for the combined similarity metric, its components and their combinations . .50

\section{Chapter III}

Figure 1 (a) Butterfly arm support board with head-neck support; (b) Example of patient positioning

Figure 2 CT images from Lung Patient \#2 showing the true and registration-derived catheter positions. Dark green and light green represent the true and reconstructed positions of the first catheter, respectively. Red and orange represent the true and reconstructed positions of the second catheter, respectively. (a) Coronal plane; (b) Transverse plane; (c) Sagittal plane; (d) 3D rendering

Figure 3 Example of x-ray data mismatch in the axial direction for Esophagus Patient 2. The green line shows catheter positions digitized from the current image, purple indicates the projected axial position for marker \#8 derived from the orthogonal image, dark red indicates the reconstructed catheter positions averaged over both images, and yellow indicates an axial mismatch of $5.5 \mathrm{~mm}$ between lateral and posterior images for marker \#8. (a) Lateral image; (b) Posterior image. 
Table 1 Registration accuracy for four esophagus and two lung cancer patients. $\mathrm{N}$ is a total number of marker positions used to compute TRE for a given patient; $\mathrm{x}, \mathrm{y}$ and $\mathrm{z}$ represent lateral, posterior-anterior and superior-inferior directions respectively; total values represent mean and maximum TREs across all marker positions for a given patient.............................64

Table 2 Algorithm precision. TOTAL value represents $\sigma_{\max }$ averaged across all patients........65

Table 3 Orthogonal data axial mismatch for each patient and resulting axial TREs. TOTAL values represent mean and maximum TREs across all patients.

\section{Chapter IV}

Figure 1 Typical CT image processed by subsequent application of MM filters: (a) Original CT image; (b) Threshold filter; (c) Holes filling filter; (d) Opening filter; (e) Median filter; (f) CT image with eliminated external objects ............................................... 83

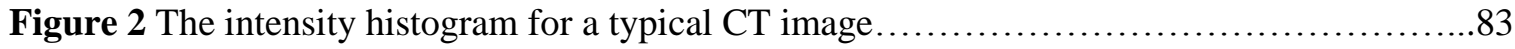

Figure 3 Example of image processing filter sequence for wire removal from CT image: (a) Section of processed CT image showing that wire was not eliminated; (b) CT image with

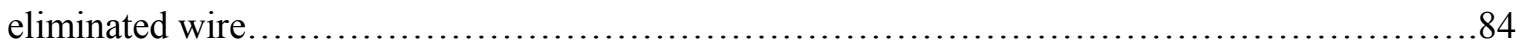

Figure 4 The sequence of images processed by MM filters for circumference extraction from a typical CT image: (a) Original CT image; (b) Pixels mapping the patient tissues; (c) Laplacian filter; (d) Overlay of extracted circumference with original CT image ........................86

Figure 5 (a) CT image in which portions of the body extend outside the field of view; (b) The segments of circumference which require correction; (c) Example of a circumference segment extrapolated with spline.

Figure 6 Example of lateral DRRs for an esophagus brachytherapy patient: (a) Generated from original CT data; (b) Generated from CT data with eliminated external objects.

Figure 7 Example of automatic marker contouring for external beam radiation therapy planning for breast treatment: (a) Solder marker; (b) Plastic filament marker .90 


\section{CHAPTER I}

\subsection{Motivation}

Lung cancer is one of the most common causes of cancer-related deaths worldwide (Dagnault et al., 2010; Zaric et al., 2010; Harms et al., 2000). The vast majority of diagnosed patients have inoperable, rarely curable disease that presents with significant side effects including coughing, hemoptysis, dyspnea, and atelectasis (Dagnault et al., 2010). Radiotherapy and chemotherapy are often offered to these patients as a palliative measure for symptom relief. In patients with luminal disease causing obstruction or bleeding, brachytherapy can be used to decrease the tumour size to open airways and stop bleeding (Celebioglu et al., 2002).

A typical high dose rate (HDR) lung brachytherapy procedure involves inserting hollow Teflon catheters into the bronchi next to the tumour location using a bronchoscope. The marker wire, which contains radio-opaque tungsten, is then placed into the catheter. Anterior-posterior and lateral x-ray images are acquired in order to localize the catheters prior to treatment. The images are transferred to a planning computer, where a treatment plan is designed by a radiation oncologist and a dosimetrist. Although, the orthogonal pair of x-ray images allows for accurate catheter reconstruction, it does not allow for the visualization of the tumour or organs at risk due to poor soft tissue contrast. As a result, the quality of treatment plans created using orthogonal images could be compromised. Since the lungs are in close proximity to the heart, spinal cord, and other critical structures, focused radiation dose delivery to the tumour is of critical importance to avoid side effects such as global respiratory failure, cardiac arrhythmia, hemoptysis, pneumothorax, pneumomediastinum, pulmonary edema, tracheoesophageal fistulae, and 
death (Harms et al., 2000). It is possible to move the patient into a CT suite to acquire 3D data with an improved soft tissue contrast, however, moving a patient with catheters in place prior to each treatment is impractical. An alternative option could be to use prior diagnostic or external beam radiation treatment planning CT images which are normally available for these patients. Currently, however, these images cannot be used for treatment planning of brachytherapy directly because of the variation in patient positioning between the $\mathrm{CT}$ and orthogonal image acquisition. In order to account for positioning differences, a $2 \mathrm{D} / 3 \mathrm{D}$ registration algorithm that registers the orthogonal images with previously acquired CT data would be needed to allow accurate localization of the treatment catheters in the CT volume.

Aligning the two data sets with acceptable accuracy would enable treatment planning to be performed in 3D, where catheters, tumour, organs-at-risk, and cardiac devices such as pacemakers could be visualized in the same view. This would allow oncologists to optimize the treatment plan for a focused dose delivery to the tumour, with minimal dose to the surrounding tissues. This has the potential to reduce the rate of complications and improve quality of life (Kim et al., 2007). Furthermore, the availability of CT data would enable the use of heterogeneity corrections to heterogeneous medium improve the dose computation accuracy, and increase the gross tumour volume (GTV) coverage. (Onal et al., 2009; Lyczek et al., 2012)

The registration of a 3D CT volume with 2D x-ray images is a well-studied problem in image-guided therapy applications. However, while there are many 2D/3D registration algorithms available, there is no single, generally accepted method suitable for all imaging modalities, sets of equipment, unique image features, and anatomical locations (Cordón et al., 2006). There are a number of challenges specific to lung brachytherapy that complicate the $2 \mathrm{D}$ to $3 \mathrm{D}$ registration process. First, the orthogonality of an image pair acquired using a c-arm system is not guaranteed since the angles are manually set, which leads to a non-negligible random angular error. Second, respiratory motion between x-ray 
image acquisitions may further decrease the fidelity of the orthogonal data set. Finally, diagnostic CT data normally does not contain objects such as the ECG leads, wires, and treatment catheters present during the orthogonal data acquisition stage, creating additional difficulties for image registration. A 2D/3D image registration algorithm that is robust to these unique characteristics of images acquired for HDR lung brachytherapy is presented in this thesis.

The algorithm is developed and optimized for the robust rigid registration of nearly orthogonal c-arm x-ray images with previously acquired CT images for HDR lung brachytherapy. The proposed algorithm uses an automated, intensity-based approach that does not require the manual definition of any image features or the introduction of external markers. The preliminary evaluation of the algorithm was performed using two tissue equivalent phantoms. The algorithm evaluation was performed in the clinical setting using data from six patients undergoing thoracic brachytherapy.

The CT images often contain external objects such as the treatment table, clothing, ECG leads, wires, and fiducial markers that are translated into the digitally reconstructed radiographs (DRRs). The presence of these objects in the DRRs may obscure useful information, which could negatively affect the quality of registration of CT data with xray images. A single CT data set may contain tens or even hundreds of axial images. Therefore, manual image filtering of external objects would be very time consuming and thus, impractical. The image processing algorithm for automatic elimination of external objects from CT images is proposed in this thesis. The proposed algorithm was later modified to extract patient circumference and correct for any irregularities and missing data in the processed images. The application of the algorithm in both radiation therapy and diagnostic radiology scenarios is demonstrated. 


\subsection{Brachytherapy}

Brachytherapy is a form of radiation therapy in which a radiation source is placed in direct proximity to the tumour or tumour bed. Brachytherapy may be used as an alternative to, or in combination with, surgery, external beam radiation, and chemotherapy. Due to the fast dose fall off, brachytherapy has the potential for a higher and more focused radiation dose delivery to the tumour compared with external beam radiation; the surrounding healthy tissues are less exposed to radiation which might reduce the risk of side effects. Brachytherapy has the potential for easy adaptation to the size and shape of the tumour. Finally, brachytherapy is less susceptible to position uncertainty resulting from tumour motion during treatment than is the case with external beam radiation ( $\mathrm{Yu}$ et al., 2011).

\subsubsection{HDR Brachytherapy}

Brachytherapy can be classified into low dose rate (LDR) and high dose rate (HDR) brachytherapy. Brachytherapy is considered to be HDR when it is capable of delivering radiation to a prescription point at dose rates greater than $20 \mathrm{cGy} /$ minute (Yu et al., 2011, Khan et al., 2009). In LDR, the prescription dose rate normally ranges from 0.5 to 2 cGy/min (Khan et al., 2009). The most common applications of HDR brachytherapy are the treatment of endobronchial lung cancer, esophagus cancer, cervical cancer, localized prostate cancer, and post-lumpectomy treatment of breast cancer (Yu et al., 2011).

Presently most HDR units use an Iridium-192 source with a high activity of $\sim 10 \mathrm{Ci}$ (370 GBq) (Khan et al., 2009). The source is normally shaped like a cylinder, with a diameter between $0.3 \mathrm{~mm}$ and $0.6 \mathrm{~mm}$ and a length between $3.5 \mathrm{~mm}$ and $10 \mathrm{~mm}$. The source is welded to the end of the flexible drive cable of matching diameter. The drive cable with attached source is also called a source wire. The source wire is directed into the patient using hollow catheters or specialized applicators. The HDR unit has a number of channels and an indexer system that directs the source wire into each channel. The channels are located on a rotating turret that allows for the alignment of the source wire with each 
channel. The source can be moved along the applicator to programmed dwell positions in precise increments using a stepper motor. The accuracy of source positioning is normally within $\pm 1 \mathrm{~mm}$ (Khan et al., 2009). The accuracy of timing is determined by dwell time resolution, which is normally not worse than $0.1 \mathrm{~s}$ (Khan et al., 2009). An example of an HDR unit and rotating turret with channels is shown in Figure 1.

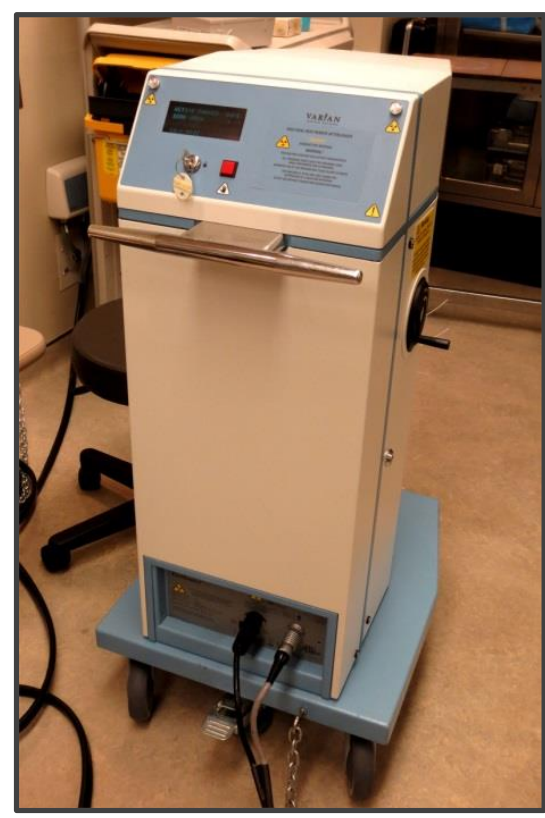

(a)

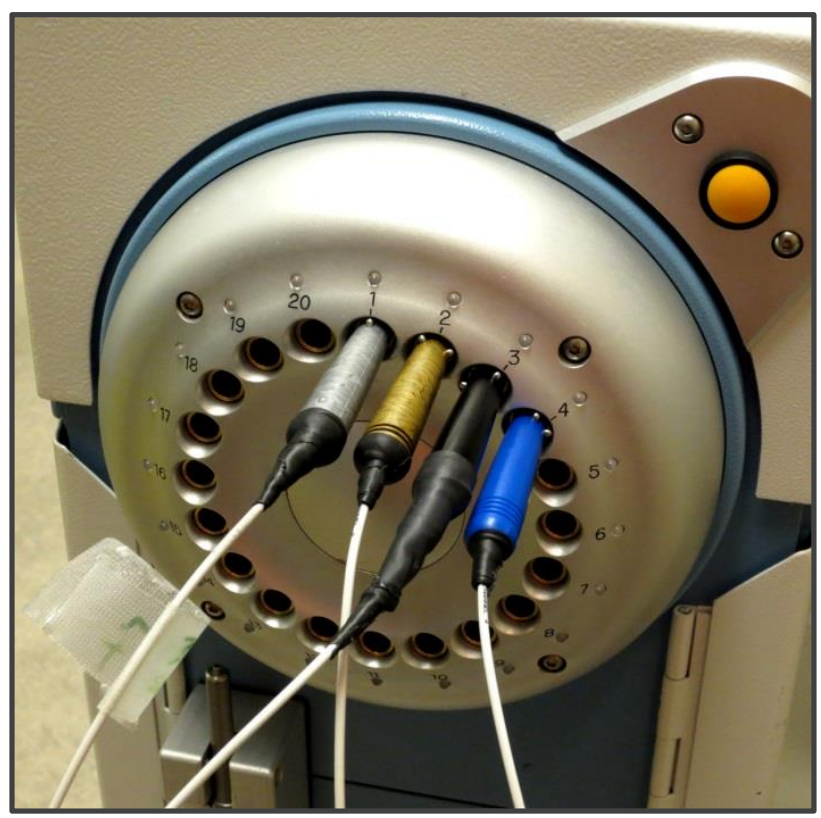

(b)

Figure 1. (a) Varian HDR unit (VariSource); (b) Rotating turret with channels.

\subsubsection{HDR Brachytherapy Treatment Planning}

Standard HDR brachytherapy treatment planning begins with patient preparation and placement of the applicators. Marker wires are then inserted into the applicators all the way to the ends and patient images acquired using a conventional c-arm x-ray unit or a CT simulator. During the isocentric c-arm x-ray simulation procedure, orthogonal radiographs are acquired allowing for the reconstruction of the marker positions. The brachytherapy team then develops a treatment plan based on the marker position in relation to the distal end of the applicator. In the case of a CT simulation, catheters are localized in 3D in relation to surrounding tissues (Khan, 2009). 
The simulation is followed by treatment planning, which begins with the transfer of acquired images into the planning computer. In the case of the c-arm x-ray simulation, the target volume is outlined in the orthogonal images. The dose specification points are also marked. In the case of a CT simulation, the acquired images can be used for treatment planning directly since they contain applicators and surrounding patient anatomy. Sliceby-slice delineation of the applicators, target volume, and organs at risk is possible. The dose distribution is then computed using the TG-43 formalism (Melhus and Rivard, 2006) or Monte Carlo (Pérez-Calatayud et al., 2005) method. Independent treatment plan verification is performed as a part of HDR quality assurance. The accuracy of the input data such as dose prescription, dwell times, and current source rate is verified (Khan, 2009). The treatment plan is then delivered as designed.

\subsubsection{Lung Brachytherapy}

Lung brachytherapy is prescribed to deliver additional radiation dose to a tumour located in the proximity of major bronchi. It is often used in combination with external radiation beam treatment as a palliative measure. Brachytherapy can shrink a tumour that is blocking the airway and help improve breathing. It may also stop the bleeding caused by the cancer (Yu et al., 2011).

The typical HDR lung brachytherapy procedure is performed as follows. The patient is consciously sedated in the HDR suite. Hollow Teflon catheters are prepared by placing a guide wire in the lumen to make them more rigid for insertion. The catheter is inserted into the patient's bronchi at the tumour location through the biopsy channel of the bronchoscope and the bronchoscope is then removed, leaving the catheter in place. The

guide wire is then replaced with a marker wire that has radio-opaque markers. This procedure is repeated if more than one catheter is required for the treatment. The anteriorposterior and lateral radiographs of the thorax are then acquired using the c-arm in order to localize the catheters. The images are sent to the planning computer where a treatment 
plan is designed. This plan is delivered to the patient using the remote afterloader. The remote afterloader can control the transfer of the radiation source into specified positions along the catheter and automatically withdraws the source after treatment. The treatment is performed in a shielded room with no staff present during the treatment. The vital functions of the patient are monitored remotely with the help of video equipment and sensors. The treatment can be interrupted remotely at any time. At the end of treatment, the catheters are removed in the HDR suite and the patient is transferred to a nursing station for observation and recovery. After a suitable recovery time, the patient is released. The typical HDR brachytherapy setup is shown in Figure 2. The typical pair of $\mathrm{X}$-ray images and the corresponding 2D-based treatment plan for a lung patient with two catheters is shown in Figure 3.

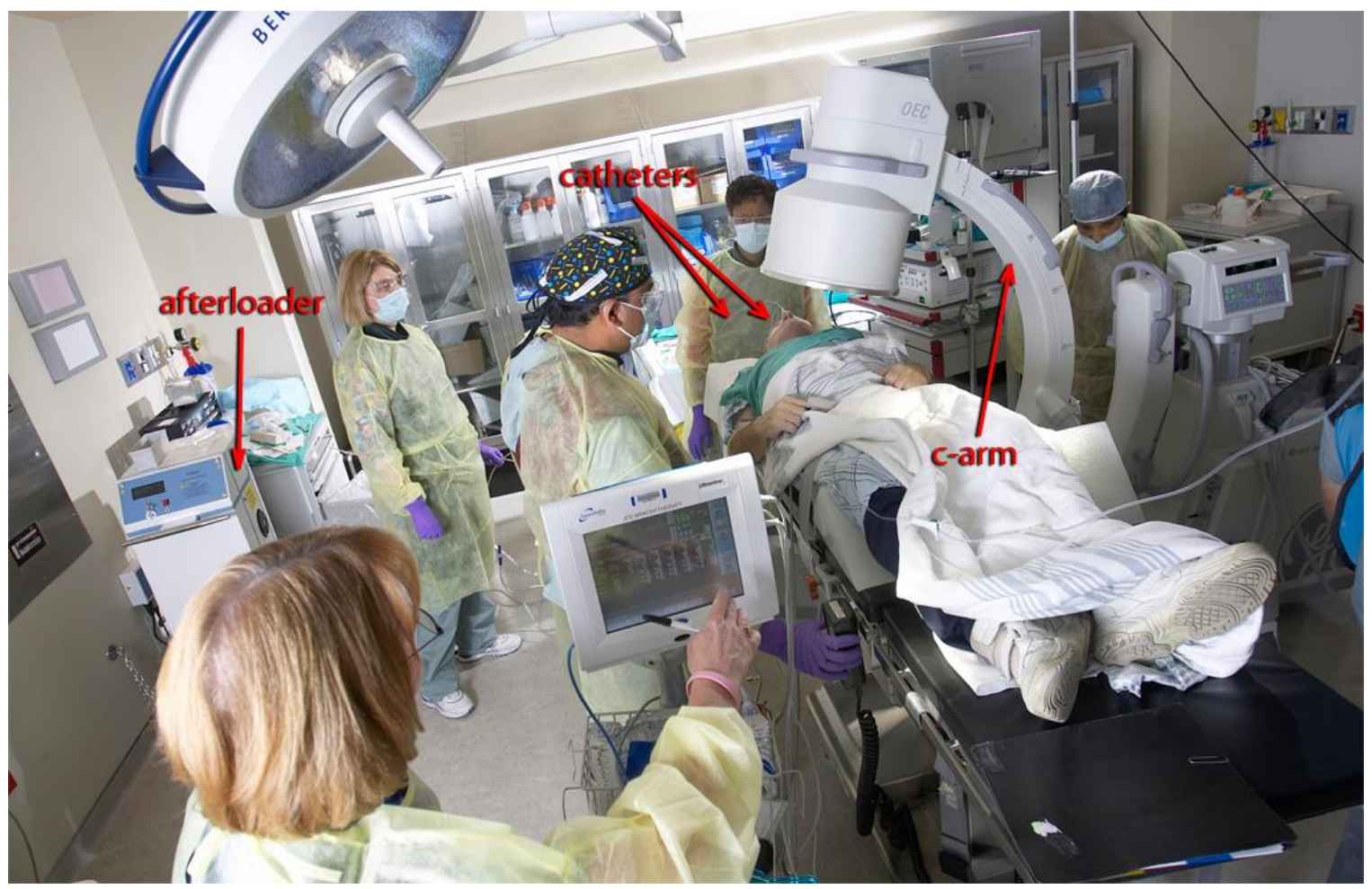

Figure 2. Typical HDR brachytherapy treatment setup showing patient with inserted catheters, c-arm, and radiation source afterloader. 


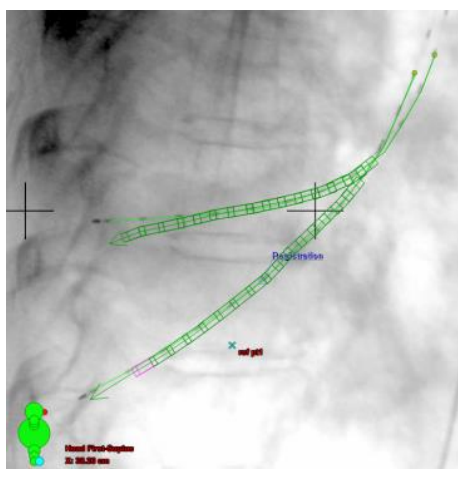

(a)

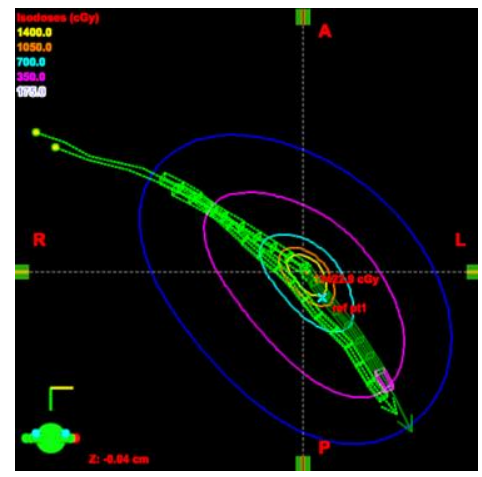

(d)

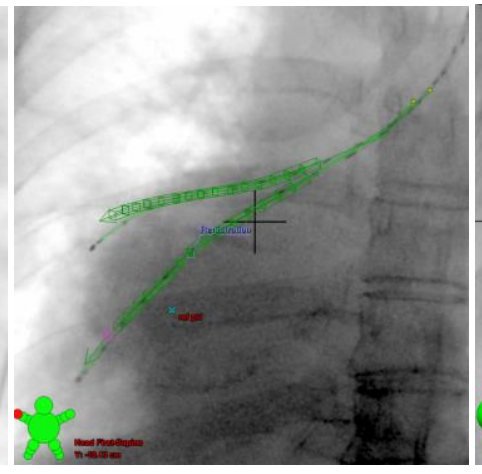

(b)

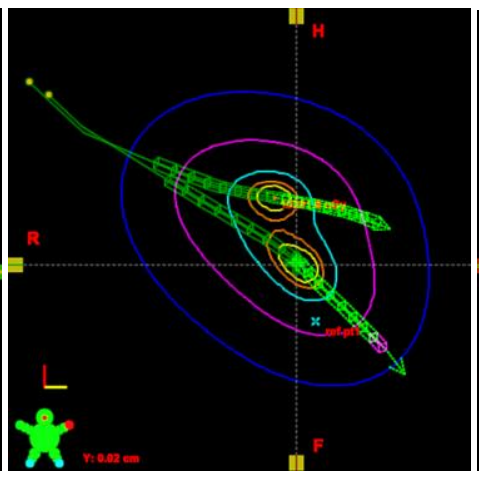

(e)

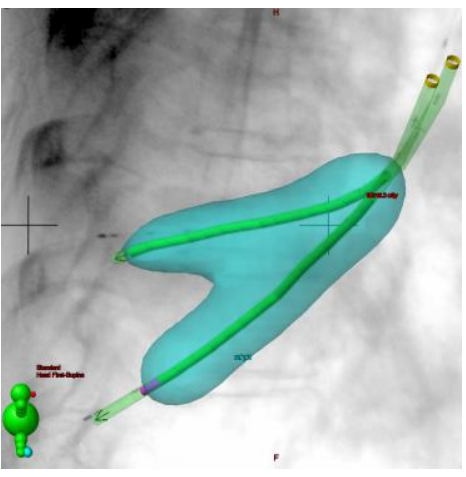

(c)

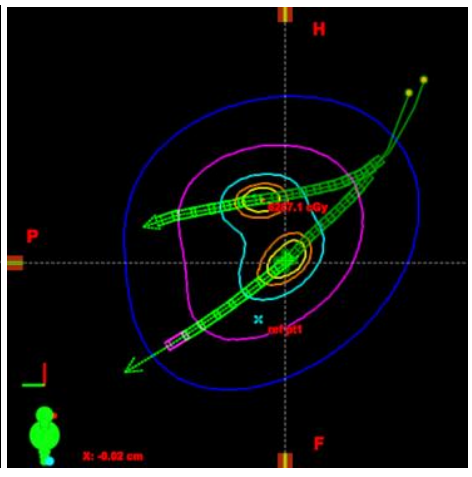

(f)

Figure 3. Typical pair of x-ray images and a lung brachytherapy treatment plan: (a) Lateral $\mathrm{x}$-ray image with digitized catheters; (b) PA x-ray image with digitized catheters; (c) 3D rendering of the treatment volume and reconstructed catheters; (d) Transverse slice dose distribution; (e) Coronal slice dose distribution; (f) Sagittal slice dose distribution.

\subsection{Image Registration}

Image registration is an important technology for image-guided therapy. The goal of image registration is to align one image with another. The process is often used to improve visibility in minimally invasive therapies. (Markelij et al., 2012).

\subsubsection{Registration Dimensionality}

In the majority of cases, pre-intervention data exists in the form of a three-dimensional (3D) CT or MRI volume. The intra-intervention data could be two-dimensional (2D) $\mathrm{x}$ ray, CT-fluoroscopy, ultrasound and optical images, or 3D such as volume ultrasound or 
cone-beam CT (Markelij et al., 2012). The registration problem could then be classified as 2D-to-3D or 3D-to-3D depending on the dimensionality of the intra-intervention data. In order to perform a 2D-to-3D registration, the data should be brought to dimensional correspondence by either converting the $2 \mathrm{D}$ data to $3 \mathrm{D}$, or by converting the $3 \mathrm{D}$ data to 2D. This can be achieved by a variety of methods reviewed by Markelij et al. (2012).

When registering a 3D CT volume with 2D x-ray images, digitally reconstructed radiographs (DRRs) must be generated from the CT volume and then associated with the available 2D images. A ray casting method (Sherouse et al, 1990) is one approach for generating DRRs. In this method, x-ray images are approximated by integrating the CT volume from the source to the pixel position on the film, simulating the process of $x$-ray propagation through the tissue. Let $V(\vec{r})$ represent CT volume that stores the original linear attenuation coefficients in Hounsfield unit (HU) scale. The original linear attenuation coefficient can then be computed using (Battista et al, 1980):

$$
\mu(\vec{r})=[(V(\vec{r}) / 1000)+1] \mu_{w}
$$

where $\mu_{w}$ is a linear attenuation coefficient of water. The intensity of the pixel at $(m, n)$ position is calculated as:

$$
\operatorname{DRR}(m, n)=I_{0} \exp \int_{0}^{L}-\mu\left(\vec{r}_{m n} \cdot l / L\right) d l,
$$

where $I_{0}$ is the original ray intensity value and $\vec{r}_{m n}$ is defined as:

$$
\vec{r}_{m n}=(w \cdot n-(W-w) / 2, h \cdot m-(H-h) / 2,-L),
$$

where $L$ is the film to source distance, $w$ and $h$ are the horizontal and vertical resolution of the DRR respectively, and $W$ and $H$ are the width and height of the DRR. The schematic of ray casting method is shown in figure 4(a). The bi-planar geometry that is often used for catheter reconstruction in lung brachytherapy is shown in figure 4(b). 


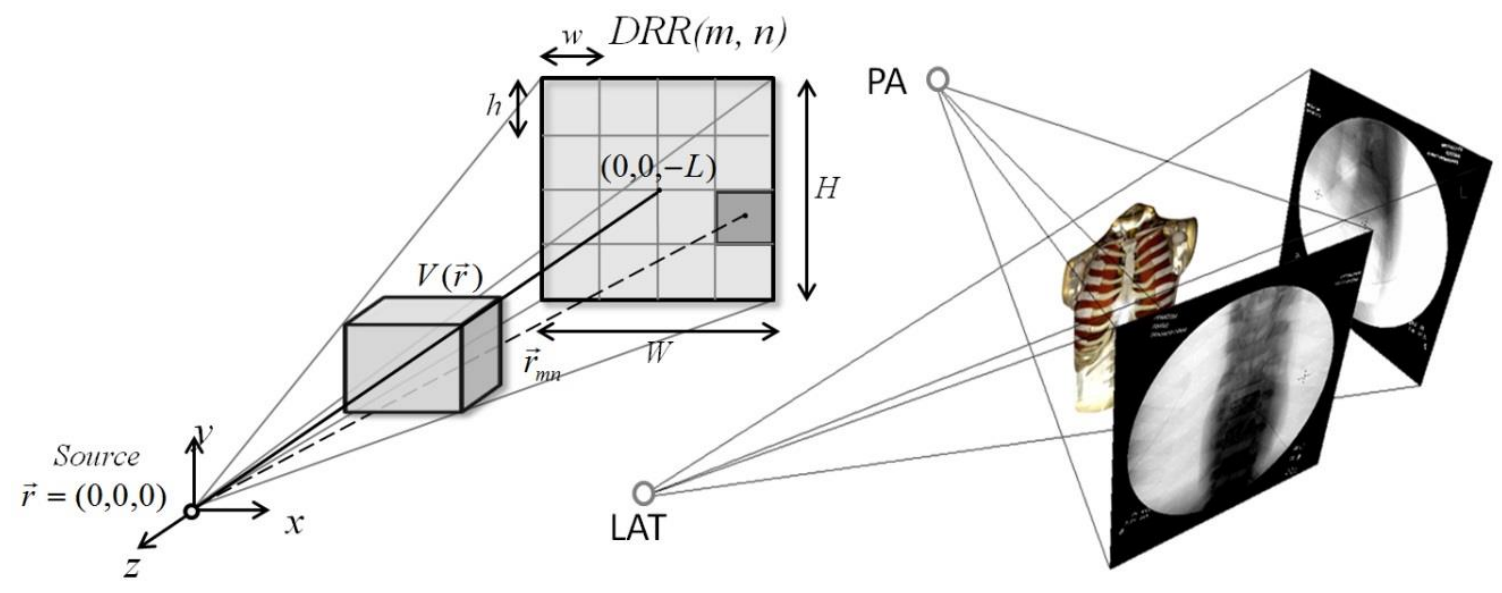

(b)

(b)

Figure 4. (a) Schematic of ray casting method; (b) Bi-planar geometry.

\subsubsection{Transformation Models}

All registration methods can be broadly divided into rigid and non-rigid methods by the nature of their spatial transformation and the number of degrees of freedoms (DOF). A rigid transformation preserves the distance between any given pair of points in Cartesian space, such that any object in space maintains its shape and size. Any rigid transformation can be decomposed into three types of transformations: translation $(\mathrm{T})$, rotation $(\mathrm{R})$, and reflection $(\mathrm{M})$. In image registration, reflections are not permitted to preserve the handedness of objects in space. In $3 \mathrm{D}$, rigid transformation is determined by six parameters $t_{x}, t_{y}, t_{z}$, and $\theta_{x}, \theta_{y}, \theta_{z}$ representing translations and rotations in threedimensional space. The parameters $t_{x}$ and $\theta_{x}$ usually correspond to patient translation in the lateral direction and rotation about the lateral axis respectively; $t_{y}$ and $\theta_{y}$ correspond to posterior-anterior translation and rotation; $t_{z}$ and $\theta_{z}$ correspond to axial translation and rotation. The transformation can then be denoted in matrix form as:

$$
T R=\left[\begin{array}{cccc} 
& & & t_{x} \\
& R & & t_{y} \\
& & & t_{z} \\
0 & 0 & 0 & 1
\end{array}\right],
$$


where $R=R_{x} \cdot R_{y} \cdot R_{z}=$

$$
\left[\begin{array}{ccc}
1 & 0 & 0 \\
0 & \cos \theta_{x} & -\sin \theta_{x} \\
0 & \sin \theta_{x} & \cos \theta_{x}
\end{array}\right] \cdot\left[\begin{array}{ccc}
\cos \theta_{y} & 0 & \sin \theta_{y} \\
0 & 1 & 0 \\
-\sin \theta_{y} & 0 & \cos \theta_{y}
\end{array}\right] \cdot\left[\begin{array}{ccc}
\cos \theta_{z} & -\sin \theta_{z} & 0 \\
\sin \theta_{z} & \cos \theta_{z} & 0 \\
0 & 0 & 1
\end{array}\right]
$$

Once all six parameters are determined and if the targeted anatomy meets the rigidity criterion, any point $(x, y, z)$ in the source data can be registered with a corresponding location $\left(x^{\prime}, y^{\prime}, z^{\prime}\right)$ in the target data using following transformation:

$$
\left(x^{\prime}, y^{\prime}, z^{\prime}, 1\right)=T R(x, y, z)=T R \cdot\left(\begin{array}{c}
x \\
y \\
z \\
1
\end{array}\right),
$$

where $\operatorname{TR}(x, y, z)$ denotes the transformation of the source point to the target (Faugeras, 1993).

The simplest non-rigid transformation that is often used in medical applications is the affine transformation. An affine transformation does not preserve the distances between points in space, but preserves the parallelism of lines. In addition to translations and rotations, affine transformations permit scaling $(\mathrm{S})$ and shearing $(\mathrm{H})$. In matrix form it can be denoted as:

$$
\begin{aligned}
& T R S H=\left[\begin{array}{cccc} 
& & & t_{x} \\
& R S H & & t_{y} \\
& & & t_{z} \\
0 & 0 & 0 & 1
\end{array}\right], \\
& R S H=R \cdot\left[\begin{array}{ccc}
s_{x} & 0 & 0 \\
0 & s_{x} & 0 \\
0 & 0 & s_{z}
\end{array}\right] \cdot\left[\begin{array}{ccc}
1 & h_{x} & h_{y} \\
0 & 1 & h_{z} \\
0 & 0 & 0
\end{array}\right],
\end{aligned}
$$


where, $s_{x}, s_{y}, s_{z}$ and $h_{x}, h_{y}, h_{z}$ are scaling and shearing factors along the $x, y$ and $z$ directions respectively. Scaling factors greater than 1 correspond to stretching, while scaling factors between 0 and 1 correspond to compression along corresponding axes. If a scaling factor is equal to 1 , no scaling occurs. An affine transformation is determined by a total of 12 DOFs (Faugeras, 1993).

Rigid or affine transformations are applied to the entire data volume, affecting all points by the same transformation. As a result, they are often referred to as global transformations. Although an affine transformation is considered to be non-rigid, the term "non-rigid" transformation more commonly refers to a local transformation in which various parts of the source data are deformed by different transformations from each other (Faugeras, 1993). Non-rigid transformations are characterized by a large number of DOF, leading to a more complex optimization procedure that requires highly correlated source and target data in order to unambiguously determine all parameters.

\subsubsection{Data Alignment Measures}

The goal of the registration is to align each point in the source data with the corresponding location in the target data by finding the correct transformation. In order to find the correct transformation, a measure that quantifies the degree of data alignment is required. A geometry-based approach is the most straightforward way to measure image alignment. The simplest geometry-based metric is the sum of the distances between the corresponding points in source $P_{i}^{\prime}$ and target $P_{i}$ data:

$$
C(T)=\sum_{i}\left\|T\left(P_{i}^{\prime}\right)-P_{i}\right\|
$$

The correct transformation $T$ is then found by minimizing the cost function (1.3.9). The optimization is normally performed iteratively by calculating the cost function at each step for a current set of transformation parameters. An optimizer then adjusts the parameters until a preset tolerance is reached (Hajnal and Hill, 2010). 
A geometry-based metric can only be employed when both the source and target data are reducible to simple structures such as set of points and/or lines. This requires the extraction of certain anatomical features from the images, which can be done either manually or through the process of segmentation. In general, feature extraction is not a trivial procedure, and for many applications it is not a viable option.

Alternatively, the quality of transformation could be estimated by measuring the degree of correlation between source and target data using information contained in pixel/voxel intensities of images. In 2D-to-3D data registration, the data are commonly brought to dimensional correspondence by extracting 2D images from the 3D volume, which reduces the problem to registering one or more pairs of 2D images. The simplest image similarity metric is a mean square difference (MSD) between corresponding pixel intensities in two images:

$$
M S D=\frac{1}{N \cdot M} \sum_{n}^{N} \sum_{m}^{M}\left(A_{n, m}-B_{n, m}\right)^{2},
$$

where $A_{n, m}$ and $B_{n, m}$ denote the intensities of the pixel $(n, m)$ for images $A$ and $B$ respectively; $N$ and $M$ represent image dimensions in pixels. The correct transformation can then be found by minimizing the MSD (Hajnal and Hill, 2010).

The MSD is an attractive metric due to its simplicity and high computation speed. However, MSD only works for registering images in which the same tissues have similar intensities in both images. Thus, the metric is only suitable for registering images from the same modality, acquired using similar protocols, which is not the case in many registration problems.

Mutual information (MI) is an often employed similarity metric for multi and monomodal registration and is reported to be among the most accurate (Wells et al., 1996; Maes et al., 1997; Meyer et al., 1997, Studholme et al., 1997). MI is based on information 
theory, and unlike methods based on correlations or differences between image intensities, the MI method does not assume a linear relationship between the intensity values of compared images (Zheng et al., 2010). The mutual information of two images $A$ and $B$ is defined as:

$$
M I(A, B)=H(A)+H(B)-H(A, B),
$$

where $H(A)$ and $H(B)$ denote marginal entropy for image $A$ and $B$ respectively, and $H(A, B)$ denotes the joint entropy of images $A$ and $B$. The maximization of MI should correspond to proper image registration.

The entropies of image $A$ and $B$ are defined as:

$$
\begin{aligned}
& H(A)=-\sum_{i=1}^{l} P\left(a_{i}\right) \log _{q}\left(P\left(a_{i}\right)\right) \\
& H(B)=-\sum_{i=1}^{l} P\left(b_{i}\right) \log _{q}\left(P\left(b_{i}\right)\right),
\end{aligned}
$$

where $q$ is the base of the algorithm, $P\left(a_{i}\right)$ and $P\left(b_{i}\right)$ are intensity histograms for image $A$ and $B$ respectively, and $l$ is the number of discrete intensity levels in image $A$ and $B$ (Pluim et al., 2003). The commonly used $q$ and $l$ values are 2 and 256 respectively. The joint entropy is defined as:

$$
H(A, B)=-\sum_{i=1}^{l} \sum_{j=1}^{l} P\left(a_{i}, b_{j}\right) \log _{q}\left(P\left(a_{i}, b_{j}\right)\right)
$$

where $P\left(a_{i}, b_{j}\right)$ is a joint histogram that represents the probability of values $a_{i}$ and $b_{j}$ occurring together. $P\left(a_{i}, b_{j}\right) \log _{q}\left(P\left(a_{i}, b_{j}\right)\right)$ is defined to be zero, when $P\left(a_{i}, b_{j}\right)=0$ (Pluim et al., 2003). 
The joint histogram is essentially a 2D array of size $l \times l$, which could be plotted as a 2D image. The joint histogram changes with the degree of image alignment. When images are correctly registered, the same anatomical tissues in both images overlap, which shows as clusters of gray values in the joint histogram image. If the intensities of the same anatomical tissues are similar in both images, the clusters will appear near diagonal in the joint histogram image. If it is not the case, the clusters will appear away from diagonal. When images are misregistered, the tissues of different types will also overlap, resulting in the emergence of additional clusters, while the intensity of the same tissue type clusters will decrease due to a reduction in the overlap of the same tissues. This is often reffered as a dispersion of the clusters in joint histogram image (Pluim et al., 2003). The joint histograms for a fluoroscopy image of thorax aligned with the rotated image of itself at varied angles are shown in Figure 5. The images in Figure 5 show the increase of dispersion with increased image misalignment.

Since entropy is a measure of dispersion of a probability distribution, the entropy of joint histogram (joint entropy) measures the degree of cluster dispersion. The correct transformation can then be found by minimizing the joint entropy. Using joint entropy as a cost function for image registration presents some challenges since this metric is very sensitive to the amount of data overlap and its content. The inclusion of marginal entropies into $\mathrm{MI}$ in equation (1.3.11) reduces such sensitivity making MI a better fit for image registration applications (Pluim et al., 2003). 


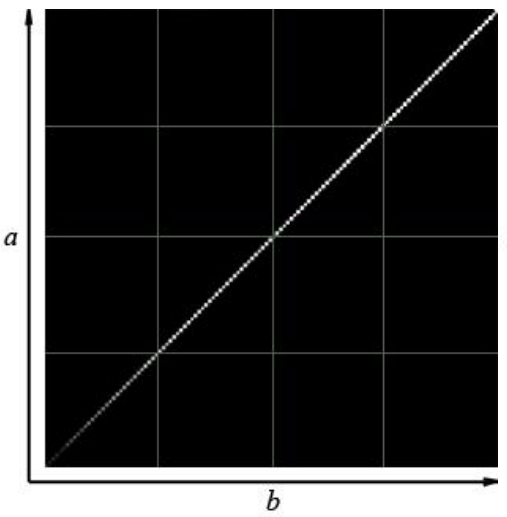

(a)

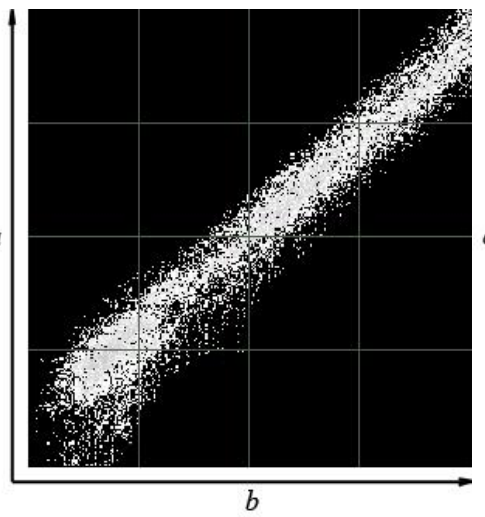

(b)

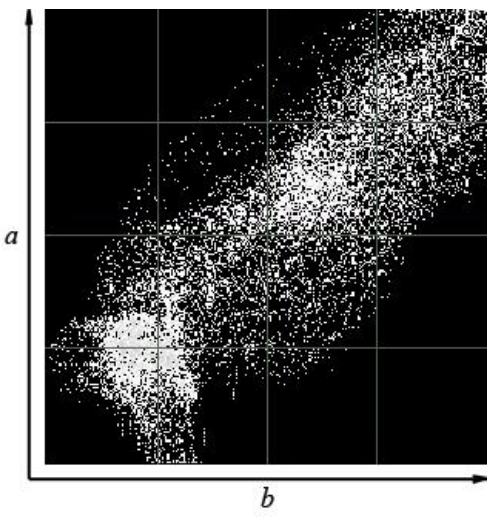

(c)

Figure 5. Joint histogram plot of the fluoroscopy image of thorax aligned with itself for different rotations: (a) no rotation; (b) $2^{\circ}$; (c) $5^{\circ}$.

It has been reported in other studies that normalized mutual information (NMI) is even less sensitive to the amount of overlap between two images compared to MI (Studholme et al., 1999). Normalized mutual information is defined as:

$$
I=N M I(A, B)=\frac{H(A)+H(B)}{H(A, B)}
$$

The major disadvantage of MI-based metrics is that they ignore valuable spatial information contained in each of the images separately (Penney et al., 1998; Zheng et al., 2010; Pluim et al., 2003).

Image gradient is another widely used similarity metric for multi- and mono-modal registration that relies on spatial information (Zheng et al., 2010; Pluim et al., 2000). The idea behind an image gradient metric is that high gradient values in images suggest transitions between different types of tissues. While the magnitude of the gradient vector might differ between different modality images, the gradient orientation should be independent of histogram variations. Therefore, gradients should point in the same or opposite directions for properly registered multimodal images (Pluim et al., 2000). A mono-modal registration gradient should point in the same direction. 
In a case of $2 \mathrm{D}$ to $2 \mathrm{D}$ registration, the gradient vector can be defined for each pixel in both images by calculating two partial derivatives in the $x$ and $y$ directions. These partial derivatives could be calculated by convolving the image intensities with a Sobel kernel of size $w \times w$. Here are the examples of most commonly used Sobel kernels of size $3 \times 3$ and $5 \times 5$ for calculating partial derivatives in $x$ and $y$ directions:

$$
\begin{gathered}
K_{x}(3)=\left[\begin{array}{lll}
-1 & 0 & 1 \\
-2 & 0 & 2 \\
-1 & 0 & 1
\end{array}\right], K_{y}(3)=\left[\begin{array}{ccc}
-1 & -2 & -1 \\
0 & 0 & 0 \\
1 & 2 & 1
\end{array}\right], \\
K_{x}(5)=\left[\begin{array}{ccccc}
-1 & -2 & 0 & 2 & 1 \\
-4 & -8 & 0 & 8 & 4 \\
-6 & -12 & 0 & 12 & 6 \\
-4 & -8 & 0 & 8 & 4 \\
-1 & -2 & 0 & 2 & 1
\end{array}\right], K_{y}(5)=\left[\begin{array}{ccccc}
-1 & -4 & -6 & -4 & -1 \\
-2 & -8 & -12 & -8 & -2 \\
0 & 0 & 0 & 0 & 0 \\
2 & 8 & 12 & 8 & 2 \\
1 & 4 & 6 & 4 & 1
\end{array}\right] .
\end{gathered}
$$

The angle between the gradient vectors for each pixel can then be calculated as:

$$
\varphi_{n, m}=\arccos \frac{\nabla A_{n, m} \cdot \nabla B_{n, m}}{\left|\nabla A_{n, m}\right| \cdot\left|\nabla B_{n, m}\right|},
$$

where $n$ and $m$ denote the pixel position on the image in the horizontal and vertical directions respectively. For mono-modal registration, the image gradient measure can be calculated by averaging the angles between gradient vectors over the entire image:

$$
G(w)=\left(\frac{1}{N \cdot M} \sum_{n}^{N} \sum_{m}^{M} \varphi_{n, m}(w)\right)^{-1},
$$

where $N$ and $M$ represent the width and height of the image in pixels respectively.

For multi-modal registration, the image gradient measure can be calculated as follows: 


$$
G(w)=\frac{1}{N \cdot M} \sum_{n}^{N} \sum_{m}^{M} \frac{\cos \left(2 \cdot \varphi_{n, m}(w)\right)+1}{2}
$$

The weighting function is introduced in equation (1.3.19) in order to equally favor $0^{\circ}$ and $180^{\circ}$ angles between gradients while penalizing angles in the middle (Pluim et al., 2000).

The correct transformation could then be found by maximizing the similarity metrics in equations (1.3.18) or (1.3.19).

When more than one pair of 2D images needs to be registered, the total cost function may be calculated by adding or multiplying similarity metrics for individual pairs of images. The multiplication approach is often favored over the addition method as it does not require similarity metric renormalization.

\subsubsection{Previous Work in 2D/3D Registration}

All 2D/3D registration methods can be divided into three major categories: intrinsic, extrinsic, and calibration-based methods. Calibration-based methods rely on a carefully pre-calibrated set of imaging equipment, where the position of the table relative to the imaging devices is tracked with sensors. In this method, the patient has to be immobilized to ensure a fixed position between data acquisitions. This can only be achieved with short times between data acquisitions. In applications with long times between pre-intervention and intervention data acquisition, the calibration-based methods are impractical.

The extrinsic methods rely on the introduction of external objects, such as fiducial markers or passive transmitters, which can be implanted directly into or next to the tumour. The markers are easily identifiable on source and target images, so the registration problem can be solved by minimizing the distance between corresponding markers in both data sets using a geometry-based alignment metric described in section 1.3.3. A number of extrinsic methods have been successfully applied to the $2 \mathrm{D} / 3 \mathrm{D}$ registration problem for tumour tracking in radiotherapy and radiosurgery (Kim et al., 
2005; Christie et al., 2005; Shirato et al., 2000; Schweikard et al., 2004). Although these methods are robust and accurate, they are invasive and put additional burden on the standard clinical procedure.

The intrinsic methods, on the other hand, rely solely on information contained in the 2D and 3D images, and do not require the introduction of external markers. These methods are divided into feature- and intensity-based methods. Feature-based methods (Hamadeh et al., 1998; Feldmar et al., 1997; Gueziec et al., 1998) rely on the registration of specific anatomical features that are extracted from images prior to registration through a segmentation process. Segmentation is a very complex step that is prone to errors and often requires user involvement (Zheng et al., 2010). The pixel/voxel intensity-based methods are by far the most reported intrinsic 2D/3D registration methods (Markelij et al., 2010). The intensity-based methods do not require segmentation and have the potential to be fully automated. In these methods, one or more of $2 \mathrm{D}$ images are simulated from 3D data and associated with available 2D images.

In the past, a large number of 2D/3D intensity-based registration methods were developed for various applications and imaging modalities. The efficient and accurate rigid registration of x-ray images with a CT volume for the skull has been reported (Chen et al., 2008; Kuduvalli et al., 2008). The rigid pelvis registration for prostate tracking was reported (Munbodh et al., 2007; Jans et al., 2006; Remeijer et al., 2000). The rigid registration of individual vertebrae was performed to assist in spinal surgery (Ho et al., 2007; Rohlfing et al., 2005; Zhang et al., 2006). The rigid x-ray image to CT volume registration of femur, knee bones and knee implants was reported (Hurvitz et al., 2008; Dennis et al., 2005), while hip implant was registered to measure its postsurgical position (Jaramaz et al., 2006; Penney et al., 2007). These methods rely on the high contrast produced by large bones and the strong rigidity associated with the registered anatomy. Thorax images contain ribs and the spinal column. These features are relatively small, deformable, and are often not in proximity $t$ the registration volume. The intensity-based rigid registration algorithm for the alignment of CT volumes with a single $2 \mathrm{D}$ axial image 
for thorax was reported (Birkfellner et al., 2007). Although it is an interesting approach, its application is limited since it is difficult to acquire an axial image using a c-arm configuration. In another study, DRRs were registered with orthogonal portal images for lung cancer radiotherapy (Künzler et al., 2007). The method employed rigid registration limited to translations only, which limits its usefulness for applications where the patient rotates between image acquisitions.

\subsection{Other Image Processing Methods}

\subsubsection{Histogram Equalization}

Prior to the calculation of the similarity metric, the images are often processed by filters in order to eliminate or enhance certain features and/or to normalize image intensities. It has been reported that the speed and accuracy of the registration is improved when both images are processed by a histogram equalization filter (Legg et al., 2007, Nam et al., 2009). Histogram equalization enhances image contrast and produces normalized image intensities. Image normalization is especially important for mono-modal registration with intensity difference-based similarity metrics. Although normalization of both images is not a requirement for multi-modal metrics such as MI and image gradient, histogram equalization enhances image contrast resulting in improved sensitivity of both metrics (Wang, 2012).

Let $h_{a}(i)$ denote an image intensity histogram, which is the probability of a pixel with intensity $i$ to occur in a gray scale image $\{a\}$. Here $0 \leq i \leq l-1$, where $l$ denotes the number of intensity levels in the image. Now, the cumulative distribution function for image $\{a\}$ is defined as (Wang, 2012):

$$
H_{a}(i)=\sum_{j=0}^{i} h_{a}(j)
$$


The goal of the histogram equalization filter is to find a new image $\{b\}$, in which the cumulative distribution function $H_{b}(i)$ is linear. This can be achieved using the following transformation for each pixel (Wang, 2012):

$$
b=H_{a}(a)
$$

The resulting image intensities are in the range of $0 \leq i \leq 1$. The conversion back to $0 \leq i \leq l-1$ can be performed using the following transformation:

$$
b^{\prime}=\lfloor b \cdot(l-1)+0.5\rfloor
$$

where \lfloor\rfloor denotes an operator that rounds down real value to the nearest integer (Wang, 2012).

An example of an image processed by an equalization histogram filter and its intensity histogram are shown in Figure 6. 


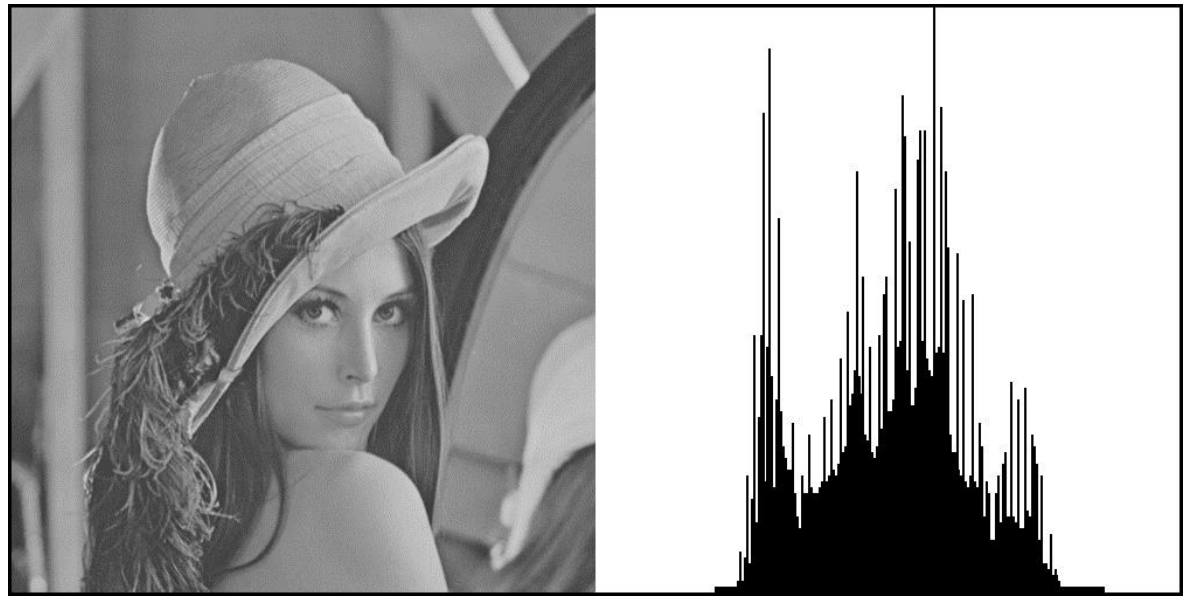

(a)

(b)

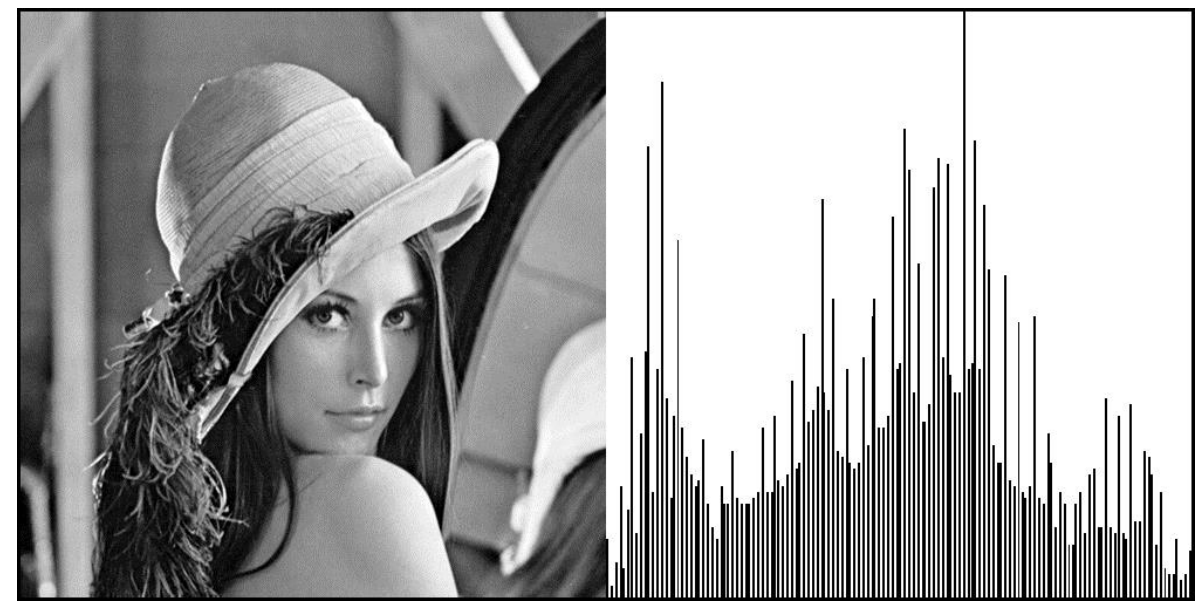

(c)

(d)

Figure 6. Example of an image processed by a histogram equalization filter: (a) Original image; (b) Intensity histogram of the original image; (c) Processed image; (d) Intensity histogram of the processed image.

\subsubsection{Mathematical Morphology Methods}

Mathematical Morphology (MM) is the theory of analyzing and processing geometrical structures, which can be applied to digital images. The MM methods (Young et al., 1998, Dougherty et al., 2003, Haralick et al., 1987, Fung et al., 2012) were originally developed for binary images, and later were extended to gray scale images. The basic idea of MM is 
to probe the image $A$ with a simple, predefined shape structuring element $B$, which is an image itself. The result of the $\mathrm{MM}$ operations is a new image $A^{\prime}=\mathrm{F}(A, B)$, where $\mathrm{F}$ denotes an MM operator. In general, the structuring element is a gray scale image of any shape and size. In each structuring element, a certain pixel is assigned to be an origin. For symmetrical structuring elements, the central pixel is chosen as the origin. The most commonly used structuring elements are 4-connected (diamond) and 8-connected (square) sets shown in Figure 7 (Young et al., 1998).

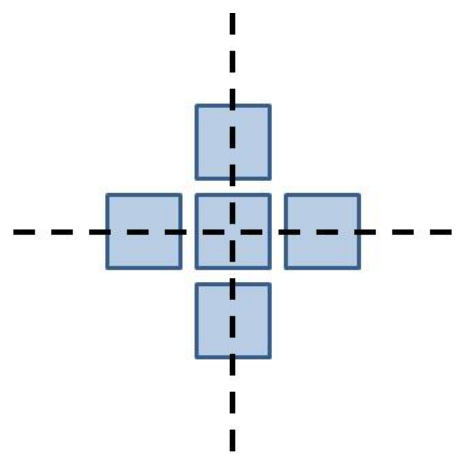

(a)

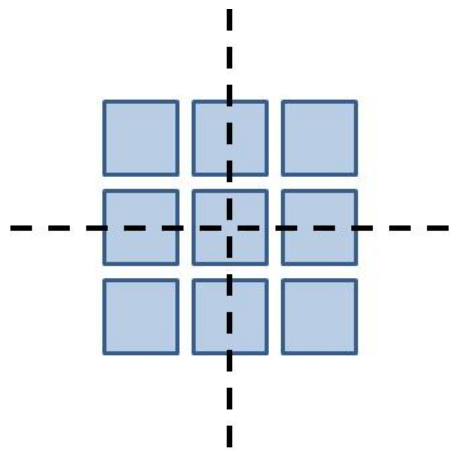

(b)

Figure 7. Standard structuring elements (a) 4-connected set; (b) 8-connected set.

In gray scale images it is important to be able to distinguish the objects from the background. The process of separating the objects from the background is called segmentation. The simplest segmentation method is called, "thresholding". In this method, the intensity level $\theta$ is selected, and applied as follows:

$$
\begin{aligned}
& \text { if } A(m, n) \geq \theta, A(m, n)=\text { object }=1 \\
& \text { if } A(m, n)<\theta, A(m, n)=\text { background }=0
\end{aligned}
$$

for bright objects in dark background. If we are interested in dark objects in bright background:

$$
\begin{aligned}
& \text { if } A(m, n) \leq \theta, A(m, n)=\text { object }=1 \\
& \text { if } A(m, n)>\theta, A(m, n)=\text { background }=0
\end{aligned}
$$


The central problem with the thresholding method is the selection of the threshold $\theta$, such that the number of pixels incorrectly determined to be an object/background is minimal. There are a number of techniques used for determining the threshold, none of which are universal or generally accepted. In many cases, the threshold is pre-determined experimentally and used independent of the data in the image (Young et al., 1998). An example of an image processed by a threshold filter is shown in Figure 8(b).

The two fundamental operators of MM are dilation and erosion. These are first-order operators, which are the basis for almost all of the higher order MM operators. The dilation is defined as:

$$
D(A, B)=\max _{[j, k] \in B}\{a[m-j, n-k]+b[j, k]\}
$$

Here, for each pixel $(m, n)$, the structuring element is summed with a shifted copy of the image, and the maximum for each combination of shifts within $B$ is used as an output. Similarly the erosion is defined as:

$$
E(A, B)=\min _{[j, k] \in B}\{a[m-j, n-k]+b[j, k]\}
$$

The dilation operator dilates the image objects by approximately a half of the structuring element size. Oppositely the erosion operator shrinks the image objects by a half of the structuring element size. The objects dilate/erode equally in all directions if $B$ is symmetrical, which is not the case for non-symmetrical structuring elements. If the sizes of the objects are smaller than the size of the structuring element, they may completely disappear from the image after erosion. Similarly, small holes within the objects could be completely eliminated by a dilation filter (Young et al., 1998). An example of an image processed by a dilation and erosion filter is shown in Figure 8(c) and Figure 8(d) respectively.

The two simplest second order operators are opening and closing defined as: 


$$
\begin{array}{ll}
\text { Opening: } & O(A, B)=D(E(A, B), B) \\
\text { Closing: } & C(A, B)=E(D(A, B), B)
\end{array}
$$

The closing operator allows for the elimination of small holes, channels and bays from the object. The opening operator removes small areas protruding from the object and thin connections between the objects. Unlike dilation and erosion, the opening and closing operators preserve the size of the objects. An example of an image processed by an opening and closing filter is shown in Figure 8(e) and Figure 8(f) respectively.

A morphological gradient is another important MM operator (Figure $(8(\mathrm{~g})$ ). It allows for the extraction of the edges of the objects, which could be useful for image segmentation. The morphological gradient is defined as:

$$
G(A, B)=\frac{1}{2}(D(A, B)-E(A, B))
$$

A morphological Laplacian is another MM operator that is commonly used for edge detection (Figure 8(h)). This operator is particularly useful for binary images when used with 4-connected structuring element as it allows for the extraction of 1 pixel thick contours of the objects. The morphological Laplacian is defined as:

$$
\begin{array}{r}
L(A, B)=\frac{1}{2}((D(A, B)-A)-(A-E(A, B)))= \\
=\frac{1}{2}(D(A, B)+E(A, B)-2 A)
\end{array}
$$

Both the Laplacian and gradient operators could be used to enhance image sharpness. The image with extracted edges could be overlaid with the original image, which would result in an improved edge sharpness of the image (Young et al., 1998). 


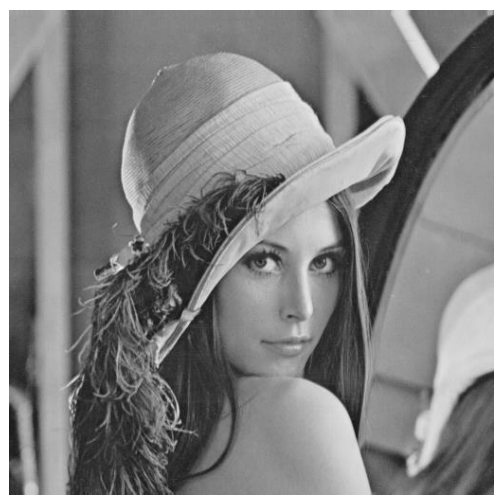

(a)

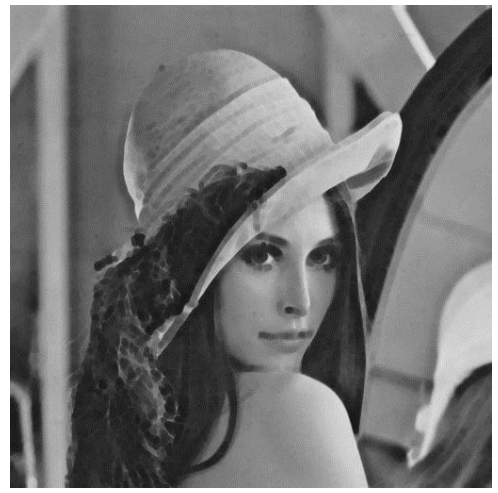

(d)

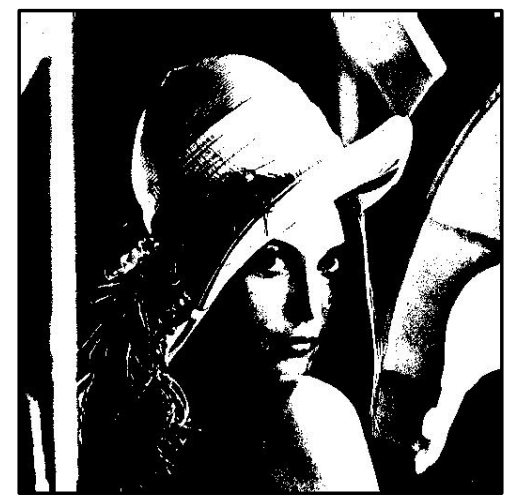

(b)

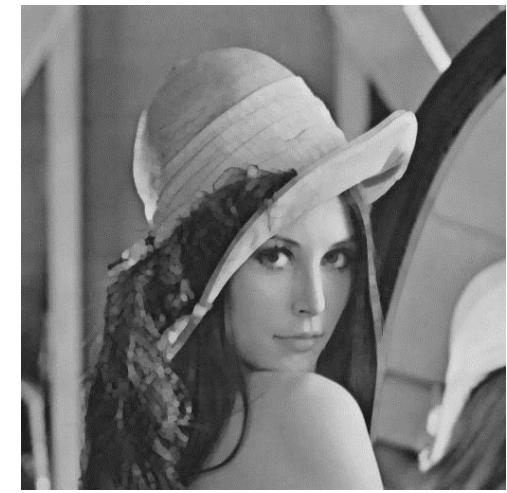

(e)

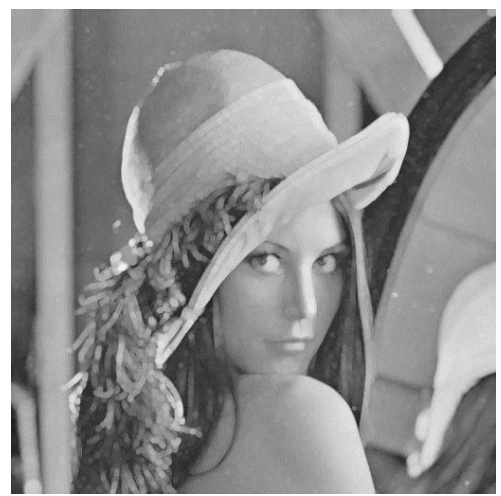

(c)

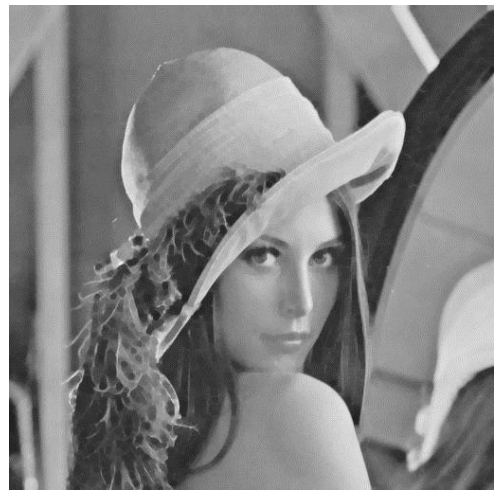

(f)

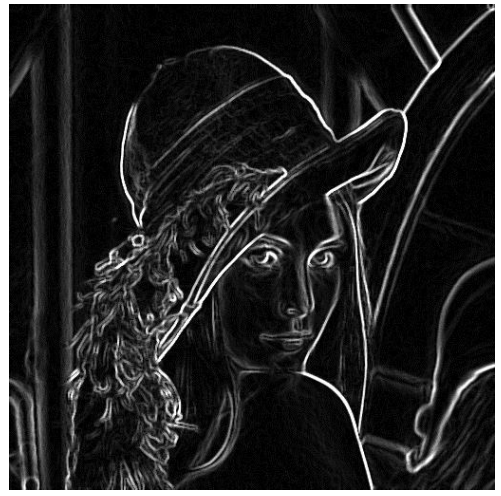

$(\mathrm{g})$

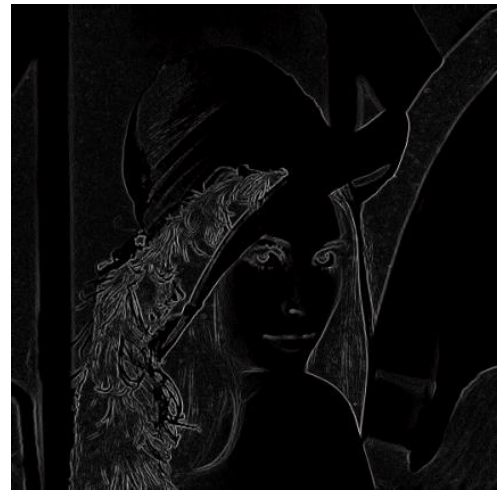

(h)

Figure 8. Example of an image processed by MM filters: (a) Original image; (b) Threshold filter (50\%); (c) Dilation; (d) Erosion; (e) Opening; (f) Closing; (g) Gradient; (h) Laplacian. 
A morphological holes filling filter fills in all holes and pits within the objects defined by the continuous edge. This filter is applied to binary images and is implemented by a recurrent application of the dilation operator with a structuring element $B$ to seed image $S^{(0)}$, which is conditioned by mask $M$ :

$$
S^{(k)}=\left\lfloor D\left(S^{(k-1)}, B\right)\right\rfloor \cap M \text { until } S^{(k)}=S^{(k-1)},
$$

where $k$ is an iteration number and $\cap$ denotes the intersection of images. With each iteration $k$ the seed image grows, but within the mask $M$. For the holes filling filter, the seed image $S^{(0)}$ is a Laplacian of the inversed original image $A$. The mask $M$ is the inverse of original image $A$ (Young et al., 1998). 


\section{CHAPTER II}

\section{PAPER I: 2D/3D registration algorithm for lung brachytherapy}

The first paper proposes an algorithm for 2D/3D registration of orthogonal x-ray images with diagnostic CT volume for high dose rate (HDR) lung brachytherapy. The algorithm utilizes a rigid registration model based on a pixel/voxel intensity matching approach. To achieve accurate registration, a robust similarity measure combining normalized mutual information (NMI), image gradient, and intensity difference was developed. The evaluation of the algorithm was performed using simple body and anthropomorphic tissue equivalent phantoms. The treatment catheters were inserted into the phantoms during data acquisition to simulate a real treatment procedure and validate the registration accuracy of the algorithm. Preliminary evaluation of the algorithm in a clinical setting was also performed.

The experiments described in this paper were performed by $\mathrm{P}$. Zvonarev, with suggestions from, and under the supervision of Dr. Farrell. The C++ code of the registration algorithm and software for algorithm evaluation was written by P. Zvonarev. The phantom data was collected by the author of this thesis, with assistance from and under the supervision of Dr. Farrell and Dr. Hunter. The patient data was collected by Dr. Sur and Emilia Timotin. The digitization of the catheters for the phantom and patient data was performed by P. Zvonarev with assistance from and under the supervision of Dr. Farrell and Dr. Hunter. The manuscript was written by P. Zvonarev and edited by Dr. Farrell, Dr. Hunter, Dr. Wierzbicki, and Dr. Hayward. The manuscript was altered from its original form to comply with the style of the thesis. 


\subsection{Paper Contents}

\section{D/3D registration algorithm for lung brachytherapy}

P. S. Zvonarev, T. J. Farrell, R. Hunter, M. Wierzbicki, J. E. Hayward and R. K. Sur Juravinski Cancer Centre and McMaster University, 699 Concession Street, Hamilton, Ontario, L8V 5C2, Canada

Medical Physics, Vol. 40, No. 2, February 2013

http://dx.doi.org/10.1118/1.4788663 


\section{Abstract}

Purpose: A 2D/3D registration algorithm is proposed for registering orthogonal $\mathrm{x}$-ray images with a diagnostic CT volume for high dose rate (HDR) lung brachytherapy.

Methods: The algorithm utilizes a rigid registration model based on a pixel/voxel intensity matching approach. To achieve accurate registration, a robust similarity measure combining normalized mutual information (NMI), image gradient, and intensity difference was developed. The algorithm was validated using a simple body and anthropomorphic phantoms. Transfer catheters were placed inside the phantoms to simulate the unique image features observed during treatment. The algorithm sensitivity to various degrees of initial misregistration and to the presence of foreign objects, such as ECG leads, was evaluated.

Results: The mean registration error was $2.2 \mathrm{~mm}$ and $1.9 \mathrm{~mm}$ for the simple body and anthropomorphic phantoms respectively. The error was comparable to the inter-operator catheter digitization error of $1.6 \mathrm{~mm}$. Preliminary analysis of data acquired from four patients indicated a mean registration error of $4.2 \mathrm{~mm}$.

Conclusions: Results obtained using the proposed algorithm are clinically acceptable especially considering the complications normally encountered when imaging during lung HDR brachytherapy.

\section{Introduction}

Lung cancer is one of the most common causes of cancer-related deaths worldwide. ${ }^{1,2,3}$ The vast majority of diagnosed patients have inoperable, rarely curable disease that presents with significant side effects including cough, hemoptysis, dyspnea, and atelectasis. ${ }^{1}$ Radiotherapy and chemotherapy is often offered to patients as a palliative measure for symptom relief. In patients with luminal disease causing obstruction or bleeding, brachytherapy can be used to shrink the tumour, providing relief of symptoms. ${ }^{4}$ 
At our institution, high dose rate (HDR) lung brachytherapy involves inserting hollow Teflon catheters through the bronchus in close proximity to tumours using a bronchoscope. Immediately prior to delivering radiation, tungsten markers are inserted into the treatment catheters and two orthogonal x-ray images are taken using a c-arm system. The markers are manually digitized to localize the catheters as a part of the treatment planning process. These images allow for accurate reconstruction of the catheter location, but do not allow visualization of the tumour or organs at risk due to poor soft tissue contrast. Since lungs are in close proximity to the heart and other critical structures, focused dose delivery to the tumour is necessary to avoid side effects such as global respiratory failure, cardiac arrhythmia, hemoptysis, pneumothorax, pneumomediastinum, pulmonary edema, tracheoesophageal fistulae, and death. ${ }^{3}$ Treatment planning in 3D would allow the radiation oncologist to optimize the delivery of radiation such that the goal of the treatment is achieved with minimal dose to normal tissue. This has the potential to reduce the rate of complications and improve quality of life. ${ }^{3,4,5}$ Furthermore, the availability of 3D data would enable to model the heterogeneous medium, improving dose computation accuracy. ${ }^{6}$

Although soft tissue is more easily identified on CT images, presently it is impractical to move a patient to a CT suite prior to each treatment due to time constraints and challenges with patient/equipment transportation. Prior diagnostic or treatment planning CT images are generally available for each patient; however, these images cannot be directly fused with the orthogonal c-arm x-ray data due to differences in patient positioning. To account for positioning differences, a 2D/3D registration algorithm that registers the orthogonal images with the diagnostic CT volume is needed to allow accurate localization of the treatment catheters in the CT volume.

The registration of a 3D CT volume with 2D x-ray images is a well-studied problem in image-guided therapy applications. Previously developed methods can be broadly divided into calibration-based, extrinsic, and intrinsic methods. ${ }^{7}$ Both calibration-based and extrinsic methods are highly impractical for our application. Intrinsic methods are 
attractive since they rely solely on images of anatomical structures, do not require external markers, and do not pose limits on the timing between 2D and 3D data acquisitions. Intrinsic techniques may be classified into feature-based methods ${ }^{8,9,10}$, intensity-based methods ${ }^{11-16}$, and gradient-based methods ${ }^{17-20}$. Feature-based methods require a segmentation stage to extract certain features and landmarks. This is not desirable in our application since image segmentation is non-trivial, often requiring user interaction $^{21}$. Intensity- and gradient-based algorithms rely on analysis of information contained in pixels and voxels of $2 \mathrm{D}$ and 3D data respectively. In intensity-based algorithms 2D images are matched with their 2D simulations from 3D data, while gradient-based methods rely on matching projected 3D gradient vector field with $2 \mathrm{D}$ image gradients. Intensity-based methods are preferred for our application due to their ability to be easily automated.

While there are many 2D-3D registration algorithms available, there is no single generally accepted method suitable for all imaging modalities, sets of equipment, unique image features, and anatomical locations. There are a number of challenges specific to lung brachytherapy that complicate $2 \mathrm{D}$ to $3 \mathrm{D}$ registration. First, the orthogonality of an image pair acquired using a c-arm system is not guaranteed since angles are set manually, leading to a non-negligible random angular error. Second, respiratory motion between $\mathrm{x}$ ray image acquisitions may further decrease the fidelity of the orthogonal data set. Finally, diagnostic CT data normally does not contain objects such as the ECG leads, wires, and treatment catheters present during the orthogonal data acquisition, creating additional difficulties for image registration. In this article we describe our 2D/3D image registration algorithm that is robust to these unique characteristics of images acquired for HDR lung brachytherapy.

The presented algorithm is developed and optimized for robust rigid registration of nearly orthogonal c-arm x-ray images with diagnostic CT images for HDR lung brachytherapy. It is understood that a general solution for lung registration problems with long delays between data acquisitions may only be achievable using deformable registration methods. 
However, an initial solution of the problem is often found using rigid registration, which is later refined using a deformable transformation model. Finding a robust initial solution is a crucial step in successful lung registration, considering the complexity of the image data. The proposed algorithm is developed using an automated, intensity-based approach that does not require the definition of any image features.

\section{Algorithm}

The proposed algorithm utilizes a rigid transformation model where registration is determined by six parameters representing translations and rotations in three-dimensional space. When all six parameters are determined, any point in orthogonal x-ray space can be registered with a corresponding point in the diagnostic CT volume.

In order to find the correct transformation, digitally reconstructed radiographs (DRRs) are generated from a diagnostic $\mathrm{CT}$ volume using a ray casting method, and then associated with the pair of orthogonal x-ray images acquired in the HDR suite. The quality of the transformation is determined by calculating a similarity metric between associated images for a given set of parameters. An optimizer then adjusts the parameters until a preset tolerance is satisfied.

Prior to the similarity metric calculation, both gray scale 4096-level orthogonal images and floating point DRR's were converted into 256-level gray scale images and then processed by a histogram equalization filter with a linearized cumulative distribution function. The value of 256 was found empirically to provide the optimal algorithm performance.

\subsection{Similarity Metric}

One of the major factors determining the registration accuracy of intensity-based methods is a similarity metric that quantifies the degree of alignment between the images. Mutual 
information (MI) is an often employed similarity measure for multi and mono-modal registration. $^{22-25} \mathrm{MI}$ is based on information theory, and unlike methods based on correlations or differences between image intensities, the MI measure does not assume a linear relationship between intensity values of compared images. ${ }^{21}$

Several studies have reported MI-based methods to be among the most accurate. ${ }^{22-25}$ The major disadvantage of MI is that while it takes into account intensity values, it ignores spatial information. It was reported that similarity measures based strictly on MI often contain an incorrect global maximum that occasionally leads to misregistration. ${ }^{15,21,26}$ In order to improve the robustness and accuracy of the proposed registration algorithm, a combined similarity measure that effectively utilizes both spatial and intensity information was proposed. The combined similarity measure is a product of three components: normalized mutual information, an image gradient measure, and an intensity difference measure.

\subsubsection{Normalized Mutual Information (NMI).}

The mutual information of two images $A$ and $B$ is defined as:

$$
M I(A, B)=H(A)+H(B)-H(A, B),
$$

where $H(A)$ and $H(B)$ denote marginal entropy for image $A$ and $B$ respectively, and $H(A, B)$ denotes the joint entropy of images $A$ and $B$. The maximization of MI should correspond to image registration.

It has been reported in other studies that NMI is less sensitive to the amount of overlap between two images compared to MI, and hence the metric was chosen for the proposed algorithm. ${ }^{27}$ Normalized mutual information is defined as:

$$
I=N M I(A, B)=\frac{H(A)+H(B)}{H(A, B)},
$$




\subsubsection{Image Gradient}

The inclusion of an image gradient term into MI-based similarity measure was proposed in several studies. ${ }^{21,28}$ This measure relies on spatial information and its incorporation into a registration algorithm can improve its robustness and accuracy. High gradient values in images suggest transitions between different types of tissues. While the magnitude of the gradient vector might differ between different modality images, the gradient orientation should be independent of histogram variations. Therefore, gradient should point in the same or opposite direction for properly registered multimodal images. ${ }^{28}$ In our case, however, both sets of data are acquired using x-ray imaging, thus, gradient direction should be the same.

The gradient vector can be defined for each pixel in both images by calculating two partial derivatives in the $x$ and $y$ directions. In the proposed algorithm, the partial derivatives were calculated by convolving image intensities with and empirically determined, $5 \times 5$ Sobel kernel.

The angle between the gradient vectors for each pixel was calculated as:

$$
\varphi_{n, m}=\arccos \frac{\nabla A_{n, m} \cdot \nabla B_{n, m}}{\left|\nabla A_{n, m}\right| \cdot\left|\nabla B_{n, m}\right|},
$$

where $n$ and $m$ denote the pixel position on the image in horizontal and vertical direction respectively. When pixel intensities within the kernel are uniform in one of the images, the denominator in equation (3) becomes zero. In this case the angle $\varphi_{n, m}$ was set to value of $\pi / 2$. The image gradient measure was calculated by averaging the angles between gradient vectors over the entire image:

$$
G(w)=\left(\frac{1}{N \cdot M} \sum_{n}^{N} \sum_{m}^{M} \varphi_{n, m}(w)\right)^{-1},
$$


where $N$ and $M$ represent the width and height of the image in pixels respectively. The inversion is required in to increase the metric as the angles between gradient vectors decrease.

\subsubsection{Intensity Difference}

The third measure, which is a mean square difference between corresponding pixels was introduced to further improve the algorithm performance:

$$
S=\sqrt{\frac{1}{N \cdot M} \sum_{n}^{N} \sum_{m}^{M}\left(A_{n, m}-B_{n, m}\right)^{2}}
$$

The purpose of the third component is to eliminate the incorrect global maxima, which may occur for the NMI and gradient measures when the registration parameters are beyond the capture range of the algorithm. The intensity difference measure effectively penalizes parameter combinations corresponding to misregistration. Unfortunately, within the vicinity of the correct solution, the intensity difference function is not stable due to variations in intensity values between two images, and may jeopardize the registration accuracy. To overcome this issue, the following weighting function was used to smooth the intensity difference measure and also favour small intensity differences:

$$
D=\gamma \frac{1}{\gamma+S^{k}},
$$

where $\gamma$ and $k$ are predefined constants that adjust the smoothness of the resulting function. The optimal values of $\gamma$ and $k$ were empirically estimated to be 0.0625 and 5 , respectively.

The proposed similarity measure was defined as a product of all three components:

$$
M=I \cdot G \cdot D
$$


Since there are two orthogonal x-ray images to be matched with corresponding DRRs in our case, the similarity measure is calculated separately for both the posteroanterior (PA) and lateral data. Then, the final registration function is calculated by multiplying the similarity metrics computed for the PA and lateral image pairs, respectively. Multiplication when constructing the final metric was favoured against summation, because it did not require normalization of terms.

\subsection{Optimization}

The best-neighbour search optimization approach smoothed by parabolic interpolation ${ }^{11}$ was chosen due to its fast convergence. In this method optimal parameter values are found iteratively. The initial search range $R_{i}(0)$, predefined for each parameter, is iteratively multiplied by a reduction factor $q_{i}$ when current parameters meet certain criteria. Here, $i$ indexes one of the six transformational parameters. For efficiency and sufficient precision, the initial search range was set to $70 \mathrm{~mm}$ and $15^{\circ}$ for translations and rotations respectively. The optimization was halted when the search range for translations dropped below $0.5 \mathrm{~mm}$. This method resulted in occasional registration failure in the early optimization steps, especially when large rotation angles were involved. Therefore, several modifications have been made to overcome the issue.

i) The search range $R_{i}(n)$ was randomly modified at each optimization step $n$, with:

$$
R_{i}^{\prime}(n)=R_{i}(n) \cdot\left(1+k_{i} \cdot \text { rand }\right)
$$

where $k_{i}$ is an empirically determined factor, and rand is a random value between -1.0 and 1.0. This modification adds a stochastic component to a purely deterministic search method, which decreases the algorithm's sensitivity to local maxima.

ii) Instead of varying all six transformation parameters from the beginning of the optimization, the following sequence was adopted. First, only translation parameters were 
varied. Once the search range for translations dropped below $40 \mathrm{~mm}$, rotation about the $y$ axis was incorporated into the optimization. Finally, after the search range dropped below $20 \mathrm{~mm}$, all six parameters were included in the optimization and the search range was reset to $40 \mathrm{~mm}$ and $10^{\circ}$ respectively.

iii) A multi-resolution approach was utilized to improve efficiency and robustness. First, both DRRs and x-ray images were resized to $128 \times 128$. When the search range for translations dropped below $40 \mathrm{~mm}, 8 \mathrm{~mm}$, and $2 \mathrm{~mm}$, the data size used was $192^{2}, 256^{2}$, and $512^{2}$, respectively.

\section{Validation}

The development and evaluation of the algorithm was performed using two tissue equivalent phantoms: a simple body phantom (QUASAR Multi-Purpose Body Phantom, Modus Medical Devices Inc., London, ON, Canada) and an anthropomorphic thorax phantom (RS-111T, Radiology Support Devices, Inc., Long Beach, CA, USA). Photographs of both phantoms are presented in figure 1. The simple body phantom had removable sections, so that objects such as treatment catheters could be inserted to mimic treatment geometry. The anthropomorphic phantom was solid, so two narrow channels of approximately $2 \mathrm{~mm}$ diameter were drilled to allow for catheter insertion.

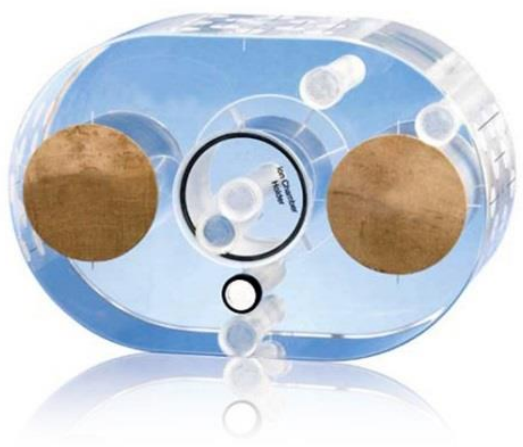

(a)

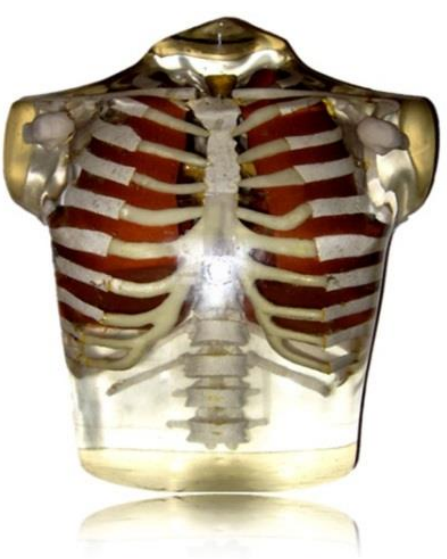

(b)

Figure 1. (a) Simple body phantom; (b) Anthropomorphic thorax phantom. 


\subsection{Similarity Metric Analysis}

The similarity metric was refined empirically to improve algorithm robustness and accuracy. First, CT images of the anthropomorphic phantom were acquired. High resolution $980 \times 980$ DRRs were generated from the acquired CT volume and used to simulate two orthogonal x-rays. Then the images were resized to the size of $512 \times 512$ to be matched with DRRs of the same size. Multiple pairs of DRRs were generated using artificially introduced transformations and matched with simulated orthogonal data sets allowing for similarity metric calculations. Each of the six transformation parameters was subsequently varied, while the other five parameters were kept at their true values yielding two-dimensional graphs of the similarity metric. The parameter variation range was $80 \mathrm{~mm}$ and $12^{\circ}$ for translations and rotations, respectively.

\subsection{Evaluation Using Simulated X-ray Images}

The initial evaluation of the algorithm was performed for both phantoms using orthogonal DRR pairs generated from CT images as in 3.1 using artificially introduced displacements. Both translations and rotations were varied randomly. The variation range was $\pm 15^{\circ}$ and $\pm 60 \mathrm{~mm}$ for rotations and translations, respectively. Simulated orthogonal pairs were then registered with the CT volume using the registration algorithm. The algorithm registration accuracy was quantified using the target registration error (TRE) ${ }^{29}$ :

$$
\operatorname{TRE}\left(P, T_{\text {reg }}, T_{\text {true }}\right)=\frac{1}{N} \sum_{i=1}^{N}\left\|T_{\text {reg }} P_{i}-T_{\text {true }} P_{i}\right\|
$$

where $T_{\text {reg }}$ is a transformation calculated by the proposed algorithm, $T_{\text {true }}$ is a true transformation required for perfect registration, and $P_{i}$ is a cloud of points depicting the $3 \mathrm{D}$ anatomical region to be registered.

Similarly, the overall degree of initial displacement $\left(T R E_{\text {init }}\right)$ was quantified using the target registration error concept: 


$$
\operatorname{TRE}_{\text {init }}\left(P, T_{\Delta}, I\right)=\frac{1}{N} \sum_{i=1}^{N}\left\|T_{\Delta} P_{i}-I \cdot P_{i}\right\|,
$$

where $T_{\Delta}$ represents transformation matrix defining the displacement, and $I$ is a unit matrix representing no transformation.

For simplicity, $P_{i}$ was an array of points evenly distributed within a sphere of $50 \mathrm{~mm}$ radius, which is a fair depiction of the registration volume important in our problem. High resolution $980 \times 980$ simulated orthogonal images were modified by a histogram filter, in order to mimic multimodal image registration and then resized accordingly to be matched with the generated DRRs.

\subsection{Evaluation Using Acquired X-Ray Images}

To evaluate the registration accuracy of the proposed algorithm under clinical conditions the following method has been developed. First, two sets of phantom CT images with and without the inserted treatment catheters $\left(\mathrm{CT}_{+}\right.$and $\left.\mathrm{CT}_{-}\right)$, were acquired while maintaining the phantom in the same position. The PTFE catheters are standard lung catheters supplied by Varian, and were $150 \mathrm{~cm}$ long and 4.7 Fr. in diameter. The CT data were reconstructed with a $1 \times 1 \mathrm{~mm}$ pixel size and $3 \mathrm{~mm}$ slice thickness. Sets of orthogonal $\mathrm{x}-$ ray pairs were collected for the phantoms with inserted catheters in place. The pixel size in the x-ray images was $0.32 \times 0.32 \mathrm{~mm}$. In order to eliminate the influence of the catheters and simulate actual diagnostic CT images, the CT. volume was used in the registration process. The catheter positions digitized from the orthogonal pair reconstruction were processed by the proposed registration algorithm and positioned in the CT. data. The transformed catheter coordinates were then compared with their true positions, which could be directly digitized from the $\mathrm{CT}_{+}$data. The registration error was quantified by computing the distances between the fused and the true catheter positions. In order to investigate the algorithm sensitivity to the amount of initial displacement, orthogonal data sets were acquired for eight different phantom positions. The phantoms 
were placed in a custom body mould to achieve reproducible positioning. The phantoms were initially positioned, so their geometrical centres were aligned with isocentre of the carm and then were translated and rotated by known amounts. The variation from the original position was up to $\pm 15^{\circ}$ and $\pm 50 \mathrm{~mm}$ for rotations and translations respectively.

\section{Results and Discussion}

\subsection{Similarity Metric Analysis}

The normalized graphs of the registration function and its components for the anthropomorphic phantom versus translations and rotations in a representative direction (x-axis) are presented in figure 2(a) and figure 2(b) respectively.

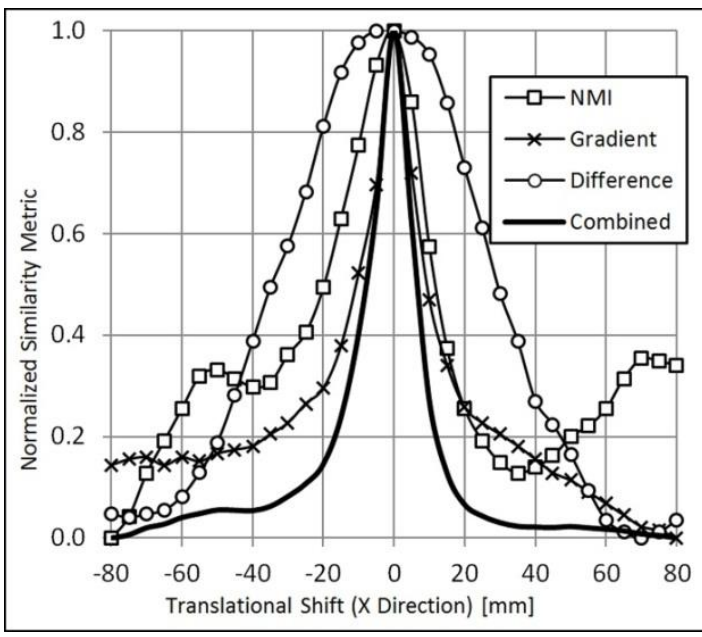

(a)

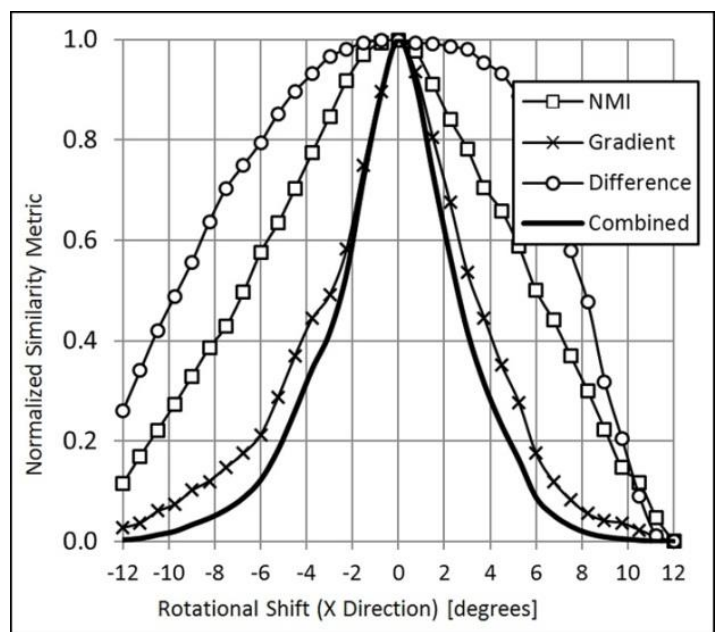

(b)

Figure 2. (a) Normalized registration function and its components vs. translational shift in the $x-$ direction; (b) normalized registration function versus rotational shift about the $\mathrm{x}$-axis.

The NMI component presented in figure 2 has a distinct registration peak both for translations and rotations. The behaviour of the function, however, is quite unstable beyond the $30 \mathrm{~mm}$ search range, which is evident for the translations shown in figure 2(a). This similarity metric contains several local maxima and has incorrect global maximum beyond those shown in the graph search range. Even though the registration 
peak is located at the proper position, successful registration depends critically on the starting search parameters. The gradient measure component has much sharper registration peaks compared to NMI, both for translations and rotations. The location of the registration peak is correct and matches the NMI for all parameters. The intensity difference component has very broad, smooth peaks compared to both NMI and gradientbased functions. Therefore, incorporation of this component into the overall registration function should not greatly affect the registration accuracy. On the other hand, this measure effectively penalizes parameter combinations distant from the correct solution, which helps to bring the search into the vicinity of the optimal solution, and eliminate the issue of incorrect global maximum.

The combined registration function incorporating all three components has a favourable shape. It has a very sharp peak located at the proper position for both translations and rotations without visible local and global maxima.

\subsection{Evaluation Using Simulated X-ray Images}

\subsubsection{Registration Accuracy / Computational Time versus Initial Displacement}

The registration accuracy and computational time sensitivity of the algorithm to the amount of initial displacement was investigated. Registration accuracy and computational

time for the anthropomorphic phantom versus the overall initial displacement $\left(T R E_{\text {init }}\right)$ is shown in figure 3. 


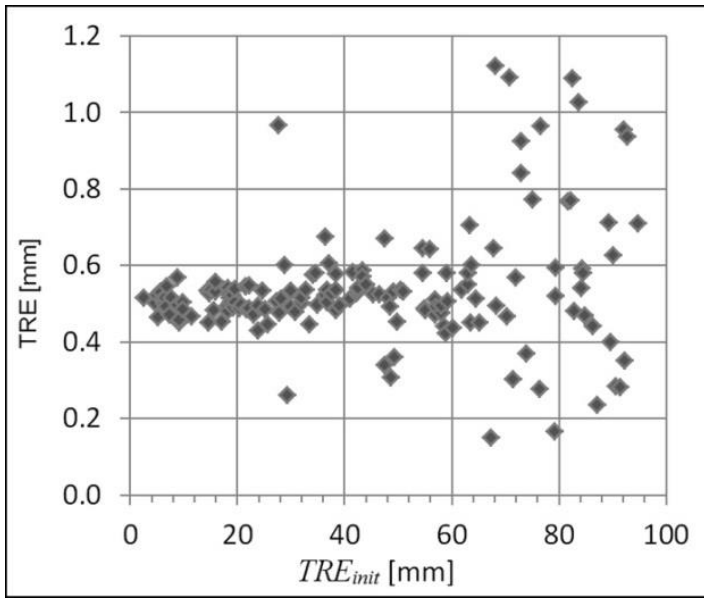

(a)

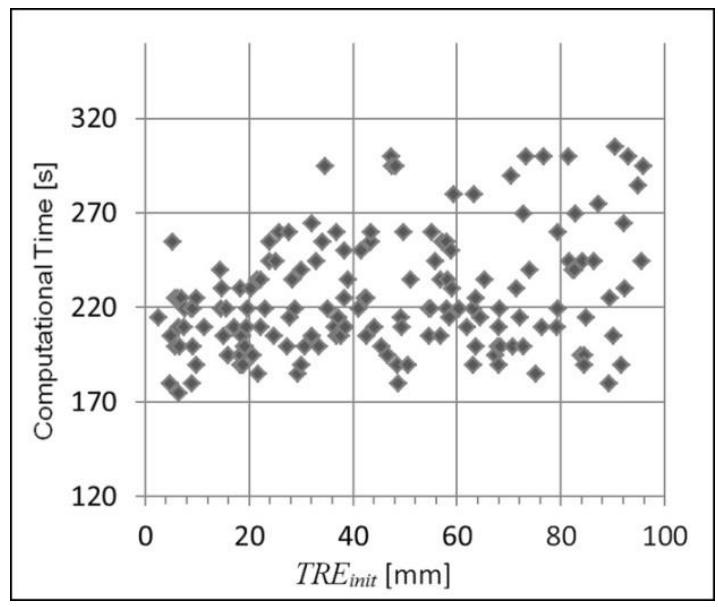

(b)

Figure 3. (a) Registration accuracy versus $T R E_{\text {init }}$; (b) Computational time versus $T R E_{\text {init }}$.

\subsubsection{Failure Rate versus Initial Displacement}

The influence of overall initial displacement $T R E_{\text {init }}$ on the algorithm robustness was evaluated. Normally, 35 to 40 iterations were sufficient to find the correct solution. The algorithm was deemed to be failing when the number of iterations exceeded 60, which indicated that the algorithm was trapped in a local maximum, or when the TRE exceeded $2 \mathrm{~mm}$, indicating a larger than expected misregistration for this simple experiment.. The algorithm failure rate with respect to $T R E_{\text {init }}$ for the anthropomorphic phantom is presented in figure 4. Results are based on 500 trials for $T R E_{\text {init }}$ evenly distributed within the range $[0,100] \mathrm{mm}$. 


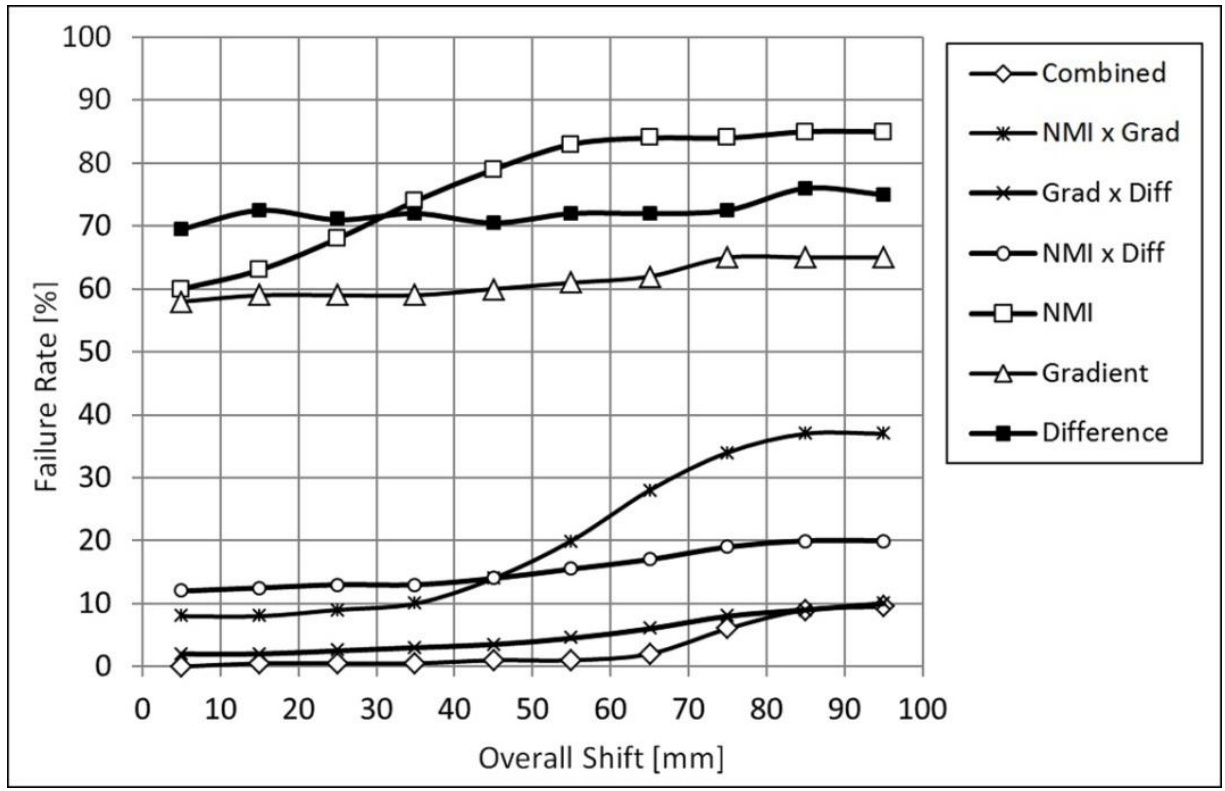

Figure 4. Algorithm failure rate versus $T R E_{\text {init }}$ for combined similarity metric and its components.

The influence of each component of the proposed similarity measure on algorithm performance in terms of registration accuracy and computational time was investigated for the data that met the convergence criteria. The results of the evaluation for the anthropomorphic phantom are summarized in table 1. The combined metric outperforms any single metric or any combination of two. All metrics have comparable computational time, and the combined metric is the most robust with virtually zero failure rate for initial displacements of up to $50 \mathrm{~mm}$. Similar trends were observed for the simple body phantom (data not shown).

\begin{tabular}{l|ccccccc}
\hline & NMI & Gradient & NMI×Grad & Diff & \multicolumn{2}{c}{ Diff $\times$ NMI } & Diff $\times$ Grad Combined \\
\hline TRE (Mean) [mm] & 0.96 & 0.65 & 0.59 & 1.29 & 0.94 & 0.60 & 0.57 \\
TRE (Maximum) [mm] & 1.88 & 1.76 & 1.75 & 1.99 & 1.83 & 1.75 & 1.73 \\
Computational Time (Mean) [s] & 271 & 242 & 239 & 298 & 265 & 240 & 227 \\
Computational Time (Max) [s] & 310 & 316 & 292 & 334 & 323 & 312 & 290 \\
\hline
\end{tabular}

Table 1. Summary of the algorithm's numerical evaluation results for the data that met the convergence criteria. 


\subsubsection{Registration Accuracy versus Geometry Error}

Another factor that may influence registration accuracy clinically is the deviation from the assumed $90^{\circ}$ angle between PA and lateral images. Since the c-arm angle is set manually, the actual angle is $90^{\circ} \pm \Delta \alpha$, where $\Delta \alpha$ represents the angular error. To evaluate the influence of $\Delta \alpha$ on registration accuracy, sets of quasi-orthogonal DRRs were generated with artificially introduced angular deviations ranging from $0^{\circ}$ to $3.5^{\circ}$. These DRRs were registered with the original CT data assuming $90^{\circ}$ geometry. The influence of angular deviation on the algorithm registration accuracy (TRE) for the anthropomorphic phantom is shown in figure 5. The overall initial displacement $T R E_{\text {init }}$ was evenly distributed between 20 and $30 \mathrm{~mm}$.

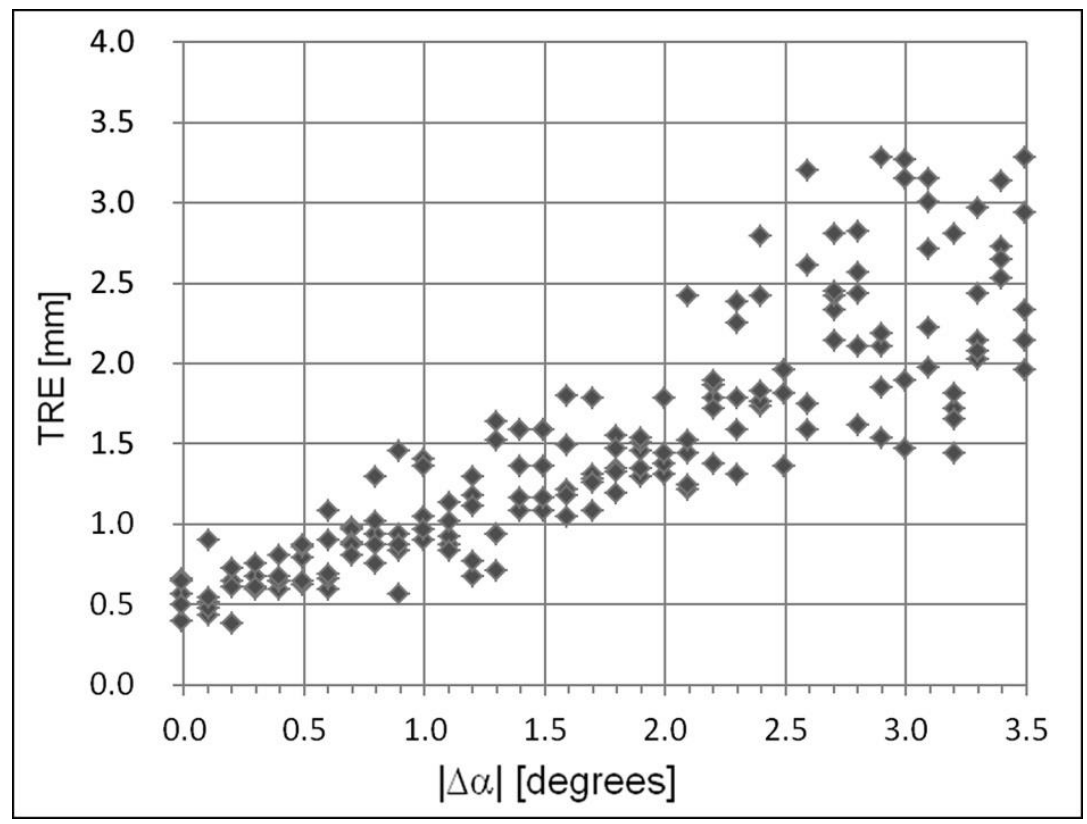

Figure 5. Registration accuracy versus angular geometry error $\Delta \alpha$.

As expected the registration error increases with angular error $|\Delta \alpha|$. The mean registration error increases from $0.6 \mathrm{~mm}$ to $1.0 \mathrm{~mm}$ for $|\Delta \alpha|$ of $\sim 1.0^{\circ}$. For angles under $3.5^{\circ}$, the failure rate of the algorithm did not increase. 


\subsection{Evaluation Using Acquired X-Ray Images}

\subsubsection{Influence of Initial Displacement on Registration}

The influence of initial displacement on registration for acquired phantom x-ray images was investigated using the procedure described in chapter 3.3. The results of the registration accuracy evaluation using $\mathrm{x}$-ray images for the anthropomorphic phantom are summarized in table 2 .

\begin{tabular}{l|cccr|crrr}
\hline & \multicolumn{4}{|c|}{ Catheter 1 } & \multicolumn{4}{c}{ Catheter 2 } \\
\cline { 2 - 9 } & $\mathrm{x}$ & $\mathrm{y}$ & $\mathrm{z}$ & Total & $\mathrm{x}$ & $\mathrm{y}$ & $\mathrm{Z}$ & Total \\
\hline TRE (Mean) $[\mathrm{mm}]$ & 0.7 & 0.8 & 1.5 & $\mathbf{1 . 8}$ & 0.6 & 0.6 & 1.8 & $\mathbf{1 . 9}$ \\
$\operatorname{TRE}($ Maximum) $[\mathrm{mm}]$ & 1.1 & 1.3 & 3.1 & $\mathbf{3 . 1}$ & 1.1 & 1.1 & 3.2 & $\mathbf{3 . 3}$ \\
\hline
\end{tabular}

Table 2. Registration accuracy evaluation for the anthropomorphic phantom.

The registration error for the anthropomorphic phantom varied from $0.5 \mathrm{~mm}$ to $3.3 \mathrm{~mm}$ for both catheters in eight unique data sets. The mean registration error was $1.9 \mathrm{~mm}$, which is approximately half the CT voxel size in axial direction.

Similar results were obtained for the simple body phantom. The registration error varied from $0.1 \mathrm{~mm}$ to $2.9 \mathrm{~mm}$. The mean registration error was $2.2 \mathrm{~mm}$, which is comparable to the results for the anthropomorphic phantom.

The algorithm failure rate for the anthropomorphic phantom is reported in table 3 . The algorithm was deemed failing when the mean TRE for one or both catheters exceeded $3 \mathrm{~mm}$. The failure rate for each metric was calculated based on a total of 40 trials: five trials for each of the eight available orthogonal data sets acquired using the procedure described in chapter 3.3 . 


\begin{tabular}{l|c}
\hline Metric & Failure Rate [\%] \\
\hline NMI & 57.5 \\
Gradient & 15.0 \\
NMl $\times$ Grad & 57.5 \\
Difference & 92.5 \\
Diff $\times$ NMI & 32.5 \\
Diff $\times$ Grad & 12.5 \\
Combined & 0 \\
\hline
\end{tabular}

Table 3. Algorithm failure rate for combined similarity metric, its components and their combinations.

The registration was successful for both phantoms in all investigated positions when combined similarity metric was used, and no correlation between the amount of initial displacement and registration accuracy was found. The largest contribution to the registration error was in the axial direction, which is explained by the lower resolution of the CT data in that direction.

\subsubsection{Influence of Foreign Bodies on Registration Accuracy}

During the acquisition of orthogonal x-rays, ECG leads were attached to the anthropomorphic phantom at typical anatomical locations and catheters were inserted. The goal was to investigate the effect of features normally not present in diagnostic or treatment planning CT data on registration accuracy. The registration with ECG leads and catheters in place was performed for the same phantom positions as in section 4.3.1., and no increase in registration error was detected. A typical example with ECG leads and catheters present is shown in figure 6. 


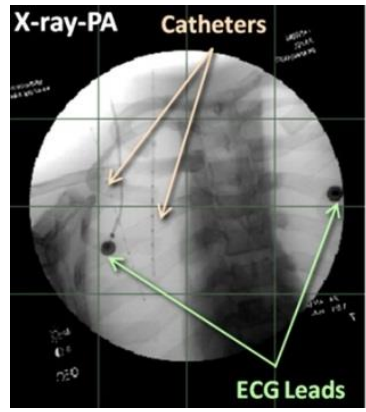

(a)

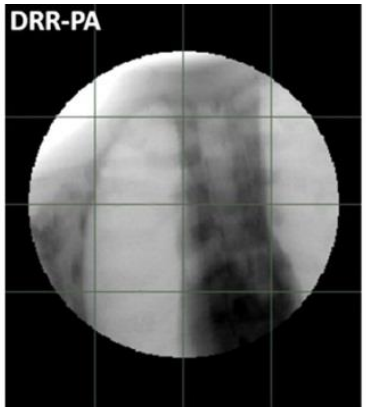

(b)

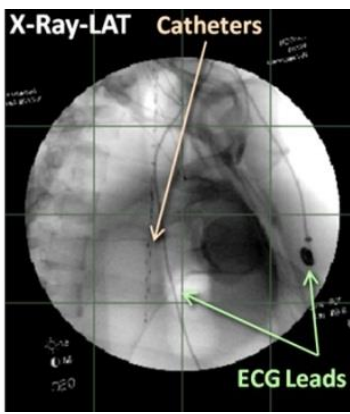

(c)

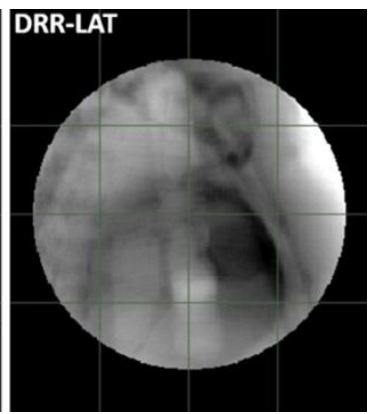

(d)

Figure 6. Example of successful registration for the anthropomorphic phantom: (a) PA x-ray image with catheters and wires; (b) Corresponding PA DRR generated from CT volume with no catheters; (c) Lateral x-ray image with catheters and wires; (d) Corresponding Lateral DRR generated from CT volume with no catheters or wires.

\subsubsection{Catheter Manual Digitization Error}

One of the major contributions to TRE, independent of registration error, is the error in identifying the radio-opaque markers both in the $\mathrm{CT}_{+}$volume and the orthogonal $\mathrm{x}$-ray images. Since digitization is performed manually by the medical physicist or dosimetrist the error is user dependent. To quantify the overall digitization error, six experienced planners were asked to digitize two catheters in the $\mathrm{CT}_{+}$volume and also in the orthogonal data sets. In order to eliminate any geometrical or registration errors from the experiment, the orthogonal data sets were generated directly from the $\mathrm{CT}_{+}$volume for the anthropomorphic phantom. The catheter positions digitized from the orthogonal images were then compared with catheters from the $\mathrm{CT}_{+}$volume digitized by the same user. The results of the digitization error estimate, averaged over six users, are summarized in table 4. These TREs are comparable to those in table 2, and the largest error occurs in the axial direction. The mean digitization error was $1.6 \mathrm{~mm}$, which is just over half a CT slice thickness. Note that the TREs in table 2 incorporate digitization, geometry, and algorithm registration error. The estimated digitization error in table 4 suggests that it is in fact a major contribution to TRE, and that we cannot expect the average TRE to be less than 1.6 $\mathrm{mm}$. Thus, the marginally higher average TRE of $1.9 \mathrm{~mm}$ in table 2 indicates the high accuracy of the proposed algorithm. 


\begin{tabular}{l|cccr}
\hline & $x$ & $y$ & $z$ & Total \\
\hline Mean TRE $[\mathrm{mm}]$ & 0.6 & 0.6 & 1.4 & $\mathbf{1 . 6}$ \\
Maximum TRE $[\mathrm{mm}]$ & 1.3 & 0.9 & 3.9 & $\mathbf{4 . 2}$ \\
\hline
\end{tabular}

Table 4. Digitization error.

\subsection{Preliminary Evaluation Using Patient Data}

Preliminary algorithm evaluation in a clinical setting was performed using one lung and three esophagus patient data sets. The patient underwent the brachytherapy procedure as usual and then orthogonal x-ray images were acquired with the catheters in place. The patient was transported to the CT simulator where the CT volume images were obtained. In this preliminary study no attempts were made to match patient positioning between $\mathrm{x}$ ray and CT data acquisitions, however the patient's arms were in the down position in both cases. No attempts to synchronize breathing phases were made. The goal was to determine the algorithm`s ability to work with real clinical data and use the result as a starting point for further algorithm improvement in addition to developing an effective data acquisition protocol. Near optimal solutions with zero failure rate were found for all four available cases demonstrating the algorithm's ability to register data containing deformable motion. The registration error varied between 1.9 and $6.5 \mathrm{~mm}$, which is reasonable considering the limits of the rigid transformation, and arbitrary patient positioning. The precision of the algorithm using these patient data was also evaluated. The original position for each patient was determined by running the algorithm with the combined similarity metric. For each patient and similarity metric the algorithm was run ten times varying the initial position up to $\pm 15^{\circ}$ and $\pm 50 \mathrm{~mm}$ for rotations and translations respectively. The resulting TREs were analyzed and the maximum standard deviation $\sigma_{\max }$ of these TREs for each patient and metric are shown in table 5. 


\begin{tabular}{l|ccccccc}
\hline & NMI & Gradient & \multicolumn{1}{c}{ NMI $\times$ Grad Difference } & \multicolumn{3}{c}{ Diff×NMI } & Diff $\times$ Grad Combined \\
\hline Lung \#1, $\sigma_{\max }[\mathrm{mm}]$ & 1.12 & 0.73 & 0.78 & 2.69 & 1.13 & 0.74 & 0.74 \\
Esophagus \#1, $\sigma_{\max }[\mathrm{mm}]$ & 1.53 & 0.91 & 0.82 & 3.51 & 1.45 & 0.87 & 0.85 \\
Esophagus \#2, $\sigma_{\max }[\mathrm{mm}]$ & 1.54 & 0.87 & 0.80 & 3.34 & 1.36 & 0.86 & 0.79 \\
Esophagus \#3, $\sigma_{\max }[\mathrm{mm}]$ & 1.44 & 0.92 & 0.96 & 3.77 & 1.47 & 0.95 & 0.96 \\
\hline Mean $\sigma_{\max }[\mathrm{mm}]$ & 1.41 & 0.86 & 0.84 & 3.33 & 1.35 & 0.86 & 0.84 \\
\hline
\end{tabular}

Table 5. Algorithm precision analysis for the combined similarity metric, its components and their combinations.

The mean standard deviation for combined metric was $0.84 \mathrm{~mm}$. The highest mean standard deviation of $3.33 \mathrm{~mm}$ was observed for the intensity difference similarity metric. From table 5 it can be seen that the precision of the combined metric was the same or better than the other metrics. However, there is insufficient patient data to determine if the combined similarity metric is more robust.

Registration results for the lung patient are shown qualitatively in figure 7. Quantitatively, the TRE ranged from 3.6 and $4.7 \mathrm{~mm}$ for this patient depending on the position along the catheter.

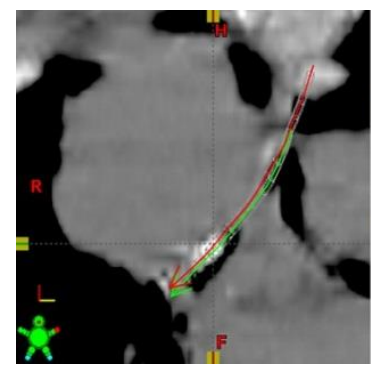

(a)

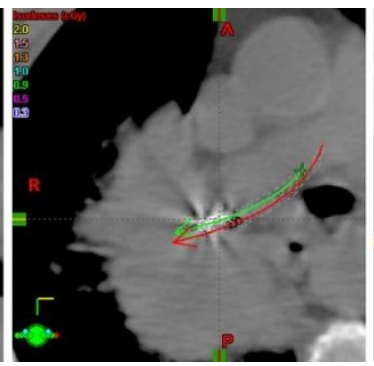

(b)

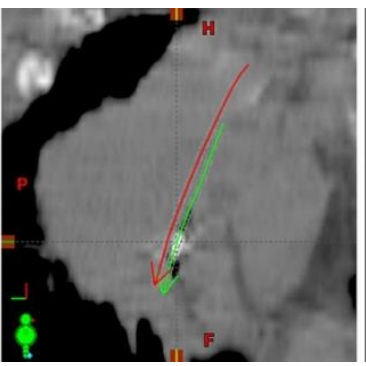

(c)

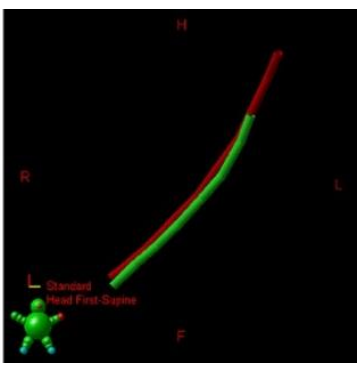

(d)

Figure 7. Diagnostic CT image slices of a lung patient showing the true (red) and registration-derived (green) catheter positions. (a) Coronal plane; (b) Transverse plane; (c) Sagittal plane; (d) 3D rendering. 


\section{Conclusions}

A 2D/3D registration algorithm for high dose rate lung brachytherapy was developed. A similarity measure combining NMI, gradient, and intensity difference was used to improve robustness and registration accuracy. Numerical evaluation of the algorithm demonstrated robust algorithm performance for various degrees of initial displacements. The average registration error was $0.54 \mathrm{~mm}$ and $0.57 \mathrm{~mm}$ for the simple body phantom and the anthropomorphic phantom respectively. The influence of geometry errors during $\mathrm{x}$-ray image acquisition on registration accuracy was evaluated numerically. It was found that registration error increases with c-arm angular deviation. The algorithm robustness was not compromised for deviations under $3.5^{\circ}$. Evaluation using actual x-ray images indicated robust registration for both phantoms in all investigated positions. The mean registration error was $2.2 \mathrm{~mm}$ and $1.9 \mathrm{~mm}$ for the simple body and anthropomorphic phantom respectively. The registration error was just over a half slice thickness and comparable to the mean digitization error of $1.6 \mathrm{~mm}$, which is clinically acceptable. The proposed registration algorithm also showed robustness to foreign bodies such as catheters and ECG-leads with wires.

As a next step in our research we will test the algorithm performance in a clinical setting. Two known challenges are expected to increase the registration error: the non-rigid nature of the patient data and patient motion during orthogonal data acquisition, mainly due to breathing. To address the issue of data non-rigidity, we plan to explore deformable registration methods and fine tune the rigid solution. To mitigate the influence of breathing on registration, we will attempt to synchronize breathing phases between orthogonal and CT data by utilizing 4D CT volume data and monitoring the respiratory signal. Finally, we intend to study the impact of the proposed method on actual doses delivered during HDR brachytherapy.

\section{Acknowledgement}

This work was supported by Varian Grant \#07-583. 


\section{References}

1. A. Dagnault, A. Ébacher, É. Vigneault, and S. Boucher, "Retrospective study of 81 patients treated with brachytherapy for endobronchial primary tumor or metastasis," Brachytherapy 9(3) 243-7 (2010).

2. B. Zaric, B. Perin, A. Jovelic, N. Lalic, N. Secen, I. Kopitovic,M. Antonic, "Clinical risk factors for early complications after high-dose-rate endobronchial brachytherapy in the palliative treatment of lung cancer," Clin. Lung Cancer 11(3) 182-6 (2010).

3. W. Harms, P. Schraube, H. Becker, D. Latz, F. Herth, P. Fritz, M. Wannenmacher, "Effect and toxicity of endoluminal high-dose-rate (HDR) brachytherapy in centrally located tumors of the upper respiratory tract," Strahlenther Onkol. 176(2) 60-6 (2000).

4. B. Celebioglu, O. U. Gurkan, S. Erdogan, I. Savas, K. Köse, C. Kurtman, U. Gonullu, "High dose rate endobronchial brachytherapy effectively palliates symptoms due to inoperable lung cancer,” Jpn. J. Clin. Oncol. 32(11) 443-8 (2002).

5. R. Y. Kim, S. Shen, J. Duan, "Image-based three-dimensional treatment planning of intracavitary brachytherapy for cancer of the cervix: dose-volume histograms of the bladder, rectum, sigmoid colon, and small bowel," Brachytherapy. 6(3) 187-94 (2007).

6. C. Onal, G. Arslan, E. Topkan, B. Pehlivan, M. Yavuz, E. Oymak and A. Yavuz, "Comparison of conventional and CT-based planning for intracavitary brachytherapy for cervical cancer: target volume coverage and organs at risk doses," J. Exp. Clin. Cancer Res. 28:95 (2009).

7. P. Markelij, D. Tomaževič, B. Likar, F. Pernuš, "A review of $3 \mathrm{D} / 2 \mathrm{D}$ registration methods for image-guided interventions," Med. Imag. Anal. 16(3) 642-661 (2010).

8. A. Hamadeh, S. Lavallée, P. Cinquin, "Automated 3-dimensional computer tomographic and fluoroscopic image registration," Comput. Aided Surg. 3 1453-62 (1998).

9. J. Feldmar, N. Ayache, F. Betting, "3D-2D projective registration of free-form curves and surfaces," Comput. Vis. Image Und. 65(3) 403-424 (1997).

10. A. Gueziec, P. Kazanzides, B. Williamson, R. H. Taylor, "Anatomy-based registration of CT-scan and intraoperative X-ray images for guiding a surgical robot," IEEE Trans. Med. Imaging 17 715-28 (1998). 
11. X. Chen, M. R. Varley, L. K. Shark, G. S. Shentall, M. C. Kirby, “A computationally efficient method for automatic registration of orthogonal X-ray images with volumetric CT," Phys. Med. Biol. 53 967-83(2008).

12. D. B. Russakoff, "Intensity-based two-dimensional-three-dimensional medical image registration," Stanford University ISBN:0-496-04390-0 (2004).

13. S. Aouadi, L. Sarry, "Accurate and precise 2D-3D registration based on X-ray intensity,” Comput. Vis. Image Und. 110 134-51(2008).

14. B. Jaramaz, K. Eckman, "2D/3D registration for measurement of implant alignment after total hip replacement,” MICCAI, LNCS 4191. 653-61 (2006).

15. Penney G P, Weese J, Little J A, Desmedt P, Hill D L, Hawkes D J 1998 A comparison of similarity measures for use in 2-D-3-D medical image registration IEEE Trans. Med. Imaging 17 586-95

16. Penney G P, Edwards P J, Hipwell J H, Slomczykowski M, Revie I, Hawkes D J 2007 Postoperative calculation of acetabular cup position using 2D-3D registration IEEE Trans. Biomed. Eng. 54(7) 1342-48

17. D. Tomaževič, B. Likar, T. Slivnik, F. Pernu`s, "3-D/2-D registration of CT and MR to X-ray images,” IEEE Trans. Med. Imaging 22 1101-9 (2003).

18. H. Livyatan, Z. Yaniv, L. Joskowicz, "Gradient-based 2-D/3-D rigid registration of fluoroscopic X-ray to CT,” IEEE Trans. Med. Imag. 22(11), 1395-1406 (2003).

19. W. Wein, B. Roeper, N. Navab, "2D/3D registration based on volume gradients," Med. Imag. 5747, 144-150 (2005)

20. P. Markelij, D. Tomaževič, F. Pernuš, B Likar, “Optimizing bone extraction in MR Images for 3D/2D gradient based registration of MR and X-ray images," Med. Imag. 6512651224

21. G. Zheng, "Effective incorporating spatial information in a mutual information based 3D-2D registration of a CT volume to X-ray images" Computerized Medical Imaging and Graphics 34(7) 553-62 (2010).

22. W. M. Wells, P. Viola, H. Atsumi, S. Nakajima, and R. Kikinis, "Multi-modal volume registration by maximization of mutual information," Med. Image Anal. 1(1) 35-51 (1996). 
23. F. Maes, A. Collignon, D. Vandermeulen, G. Marchal, and P. Suetens, "Multimodality image registration by maximization of mutual information," IEEE Trans. Med. Imag. 16(2) 187-98 (1997).

24. C. R. Meyer, J. L. Boes, B. Kim, P. H. Bland, K. R. Zasadny, P. V. Kison, K. Koral, K. A. Frey, and R. L. Wahl, "Demonstration of accuracy and clinical versatility of mutual information for automatic multimodality image fusion using affine and thinplate spline warped geometric deformations," Med. Image Anal. 1(3) 195-206 (1997).

25. Studholme C, Hill D L G, and Hawkes D J 1997 Automated three-dimensional registration of magnetic resonance and positron emission tomography brain images by multiresolution optimization of voxel similarity measures Med. Phys. 24(1) 25-35

26. J. P. W. Pluim, J. B. A. Maintz, M. A. Viergever, "Mutual-information-based registration of medical images: a survey," IEEE Trans. Med. Imag. 22(8), 986-1004 (2003).

27. C. Studholme, D. L. G. Hill, and D. J. Hawkes, “An overlap invariant entropy measure of 3D medical image alignment," Pattern Recognit. 32(1) 71-86 (1999)

28. J. P. W. Pluim, J. B. A. Maintz, and M. A. Viergever, "Image registration by maximization of combined mutual information and gradient information," IEEE Trans. Med. Imaging 19, 809 -814 (2000).

29. V. D. Kraats, G. P. Penney, D. Tomazevic, T. V. Walsum and W. J. Niessen, "Standardized Evaluation Methodology for 2D/3D Registration," IEEE Trans. Med. Imaging 24 1177-89 (2005). 


\section{CHAPTER III}

\section{PAPER II: Clinical evaluation of a 2D/3D registration algorithm for lung brachytherapy}

The second paper evaluates the proposed 2D/3D registration algorithm (Paper I) in a clinical setting using data collected from six patients undergoing thoracic brachytherapy. The imaging protocol was developed for reproducible patient setup between CT volume and orthogonal x-ray image acquisition. The oblique x-ray images were collected in addition to standard anterior/posterior and lateral images in order to investigate if their incorporation into the algorithm would improve registration quality.

The experiments described in this paper were performed by P. Zvonarev, with suggestions from and under the supervision of Dr. Farrell. The data acquisition protocol was developed by Dr. Farrell and Dr. Hunter in consultation with Dr. Sur. The patient data was collected by Dr. Sur and Emilia Timotin. The data analysis was performed by P. Zvonarev. The manuscript was written by P. Zvonarev and edited by Dr. Farrell, Dr. Hunter, Dr. Wierzbicki, and Dr. Hayward. The manuscript was altered from its original form to comply with the style of the thesis. 


\subsection{Paper Contents}

\section{Clinical evaluation of a 2D/3D registration algorithm for lung brachytherapy}

P. S. Zvonarev, T. J. Farrell, R. Hunter, M. Wierzbicki, J. E. Hayward and R. K. Sur

Juravinski Cancer Centre and McMaster University, 699 Concession Street, Hamilton, Ontario, L8V 5C2, Canada

Submitted to Brachytherapy on June 15, 2013 
Purpose: To clinically evaluate a 2D/3D registration algorithm for aligning orthogonal $\mathrm{x}$-ray images acquired during high dose rate (HDR) lung brachytherapy with a previously acquired diagnostic CT volume. Accurate image alignment would allow simultaneous visualization of the catheters, tumour and organs at risk in 3D. This would enable treatment optimization for focused radiation dose delivery to the tumour with minimal damage to the surrounding tissue.

Methods: A previously developed rigid registration algorithm based on a pixel/voxel intensity matching approach was used. This approach employs a robust similarity measure combining normalized mutual information (NMI), image gradient, and intensity difference to improve accuracy and precision. The algorithm was validated using data from four esophagus and two lung cancer brachytherapy treatments. These data capture anatomical deformations and patient motion occurring between the acquisition of the $\mathrm{x}$ ray images and $\mathrm{CT}$ volume. These inconsistencies present a significant challenge compared to the phantom images used in our previous validation.

Results: The algorithm converged to a reasonable solution for all six patient datasets. The mean and maximum registration errors were $3.2 \mathrm{~mm}$ and $6.1 \mathrm{~mm}$, respectively. The precision of the algorithm was quantified by repeating the analysis with various starting parameters. The standard deviation of the registration error was calculated to be $0.8 \mathrm{~mm}$. The algorithm was the least accurate in the axial direction, most likely due to the lower resolution of CT data in that direction and the craniocaudal motion of the diaphragm due to breathing.

Conclusions: The algorithm was deemed successful despite the presence of various anatomical deformations, patient misalignments, and data acquisition inaccuracies. The mean error was $3.2 \mathrm{~mm}$, which is acceptable considering the complexities of thoracic brachytherapy. 


\section{Introduction}

High dose rate (HDR) brachytherapy is a widely used technique for the treatment of obstructive lung cancer. In patients with luminal disease causing obstruction or bleeding, brachytherapy can shrink the tumour to relieve symptoms. ${ }^{1}$ At our institution, HDR lung brachytherapy is performed by inserting one or more hollow Teflon treatment catheters into the diseased bronchus using a bronchoscope. Immediately prior to treatment a wire with tungsten markers is inserted into the catheter and a pair of orthogonal x-ray images is acquired using a c-arm system. The markers are then manually digitized from the $\mathrm{x}$-ray images to localize catheter positions and a treatment plan is created. The limitation of this approach is that while orthogonal images allow for effective reconstruction of catheter positions, the tumour and organs at risk are often not visible due to poor soft tissue contrast. As a result, the quality of treatment plans created using orthogonal images could be compromised. The lungs are in close proximity to the heart and other critical structures; therefore, precise dose delivery to the tumour is important to minimize side effects. It is possible to move the patient into a CT suite to acquire 3D data with improved soft tissue contrast. However, moving a patient prior to each treatment is impractical. An alternative solution is to use prior diagnostic or treatment CT images which are normally available for these patients. To use these data, a 2D/3D registration algorithm capable of aligning orthogonal data with CT images is needed. Aligning both data sets with acceptable accuracy would enable 3D treatment planning, where the catheters, tumor, organs at risk and cardiac devices such as pace-makers could be simultaneously visualized. This would allow the radiation oncologist to optimize the treatment plan ensuring that the dose is delivered to the tumour with minimal dose to surrounding tissues. $^{2}$ Furthermore, the availability of 3D data would enable heterogeneous dose computation, leading to improved gross tumor volume (GTV) coverage. ${ }^{3,4}$

The registration of a 3D CT volume with 2D x-ray images is a well-studied problem in image-guided therapy applications. The use of fiducial markers implanted directly into a lung tumour has been reported in image-guided radiosurgery and radiotherapy. ${ }^{5-8}$ These 
methods rely on the introduction of external markers and are referred to as extrinsic methods. ${ }^{9}$ Although extrinsic methods are accurate, they are highly impractical for our application as they require marker implantation that places additional burden on the clinical procedure. Intrinsic methods however rely solely on information contained in 2D and 3D images and do not require introduction of external markers. These methods are divided into feature and intensity-based methods. Feature-based methods ${ }^{10-12}$ rely on registration of specific anatomical features that are extracted from images prior to registration through a segmentation process. The segmentation is a complex step that is prone to errors, and often require user involvement. ${ }^{13}$ The pixel/voxel intensity-based methods $^{14-29}$ are the most reported intrinsic $2 \mathrm{D} / 3 \mathrm{D}$ registration methods. ${ }^{9}$ In these methods one or more 2D images are simulated from 3D data and associated with available 2D images. The intensity-based methods do not require segmentation and have the potential to be fully automated.

In the past, a large number of 2D/3D intensity-based registration methods were developed. ${ }^{16-29}$ The efficient and accurate rigid registration of x-ray images with a CT volume for the skull was reported. ${ }^{16,17}$ Similar approaches were described for rigid pelvis registration to enable prostate tracking ${ }^{18-20}$ and for individual vertebrae to assist in spinal surgery. ${ }^{21-23}$ Hurvitz et al. and Dennis et al. proposed a rigid registration algorithm for images of the femur, knee bones and knee implants ${ }^{24,25}$, while Jaramaz and Eckman and Penney et al. used registration to measure the postsurgical position of hip implants. ${ }^{26,27}$ These approaches rely on the strong image contrast produced by large bones and the rigid nature of the described anatomy. Thorax images contain ribs and the spinal column but these features are relatively small, deformable, and far away from the treatment area. An intensity-based rigid registration algorithm for aligning a thorax CT volume with a single $2 \mathrm{D}$ axial image has been reported. ${ }^{28}$ This is an interesting approach but is not useful in our application since it is impossible to acquire an axial image using a c-arm configuration. In another study, Digitally Reconstructed Radiographs (DRRs) were registered with orthogonal portal images for lung cancer radiotherapy. ${ }^{29}$ The method 
employed translations only, limiting its usefulness in our application where the patient is significantly rotated between image acquisitions.

In our previous study we presented a 2D/3D registration algorithm which can register a CT image with $\mathrm{x}$-ray images acquired during lung brachytherapy. ${ }^{30}$ The algorithm used a rigid registration model and was based on a pixel/voxel intensity matching approach. To achieve accurate and robust registration, a similarity metric combining normalized mutual information (NMI), image gradient, and intensity difference was developed. In order to find the correct transformation, digitally reconstructed radiographs (DRRs) were generated from a diagnostic CT volume using a ray casting method, and then associated with the pair of orthogonal x-ray images acquired in the HDR suite. The quality of the transformation was determined by computing a similarity metric between associated images for a given set of parameters. An optimizer then adjusted the parameters (translation and rotation coordinates) until a preset tolerance was satisfied. The preliminary algorithm evaluation was performed using two tissue equivalent phantoms. The mean registration error was $2.2 \mathrm{~mm}$ and $1.9 \mathrm{~mm}$ for the simple body and anthropomorphic phantoms, respectively. These errors were comparable to the interoperator catheter digitization error of $1.6 \mathrm{~mm}$. Furthermore, the algorithm showed robustness to geometric imperfections and to the presence of foreign objects such as catheters, wires and ECG leads.

In this paper we evaluate the performance of the algorithm in a clinical setting. There are a number of challenges associated with the registration of real patient data. First, unlike in the case of phantoms, thorax anatomy deforms considerably between acquisitions due to variations in patient positioning and respiratory motion. Furthermore, the fidelity of the orthogonal x-ray data itself may be compromised by respiratory motion between lateral and anterior-posterior $\mathrm{x}$-ray image acquisition. This study evaluates how deformations between image acquisitions affect algorithm accuracy and robustness. 


\section{Method}

Although the algorithm was originally developed for intraluminal lung brachytherapy, data obtained for a mixture of lung and esophagus patients were used for the clinical evaluation. Patients were transported from the HDR brachytherapy suite to the CT simulator with the catheters in place. Only stable patients who could be safely transported between the brachytherapy suite and CT simulator were selected by the radiation oncologist. In general, esophagus patients are more stable than lung patients and therefore it was felt that the addition of this population would increase accrual. In addition, since intraluminal esophagus brachytherapy is performed using the same techniques, generally at locations close to the bronchi, these patients present the same problems in terms of marker localization and respiratory motion and are equally valid to use for algorithm validation. Research Ethics Board (REB) approval was received prior to patient data collection for the study.

At our institution, the typical HDR lung brachytherapy procedure is performed as follows. The patient is consciously sedated in the HDR suite. Hollow Teflon catheters are prepared by placing a guidewire in the lumen to make them more rigid for insertion. The $4.7 \mathrm{Fr}$ catheters are supplied by Varian (Palo Alto, CA) and are normally $150 \mathrm{~cm}$ long. The catheter is inserted into the patient's bronchus through the biopsy channel of the bronchoscope and the bronchoscope is removed, leaving the catheter in place at the tumour location. The guide wire is then replaced with a marker wire that has radioopaque tungsten markers spaced $1 \mathrm{~cm}$ apart. This procedure is repeated if more than one catheter is required for the treatment. The anterior-posterior and lateral radiographs of the thorax are acquired using a c-arm system (GE OEC 9800 Plus, GE Medical Systems) and sent to the planning computer where the catheters are manually digitized and a treatment plan is developed. This plan is delivered using the Varian VariSource (Palo Alto, CA) afterloader. At the end of treatment the catheters are removed in the HDR suite and the patient is transferred to a nursing station for observation and recovery. 
In this study, at the conclusion of treatment the patient was re-imaged using the c-arm system and then transferred to the CT suite for imaging with the catheters and the marker wires still in place. To improve setup reproducibility between the HDR suite and the CT simulator the patient was placed on the treatment table in a hands-up position with the arms supported using a polycarbonate "butterfly" board typically used for external beam breast treatment. The patient's head was placed over a foam head-neck support of matching size. The setup is shown in figure 1.

An additional part of the study was to investigate whether the incorporation of oblique $\mathrm{x}$ ray images into the algorithm would improve registration quality. Hence, in addition to the standard anterior-posterior and lateral images, a pair of oblique images at $30^{\circ}$ and $45^{\circ}$ was acquired. To eliminate any interference of the study with the normal brachytherapy procedure and to reduce the effects of any patient motion, all four images required for the study were obtained immediately after the treatment. The patient was then transported to the CT simulator where the CT data, with catheters in place, was obtained. To eliminate the chance of marker wire migration during patient transport, the wire was secured with tape at the end of the catheter. The patient breathed freely during image acquisition; no attempt to synchronize breathing phases between $\mathrm{x}$-ray and CT data was made. The CT data were reconstructed with a $1 \times 1 \mathrm{~mm}$ pixel size and $3 \mathrm{~mm}$ slice thickness. The pixel size in the $\mathrm{x}$-ray images was $0.32 \times 0.32 \mathrm{~mm}$. 


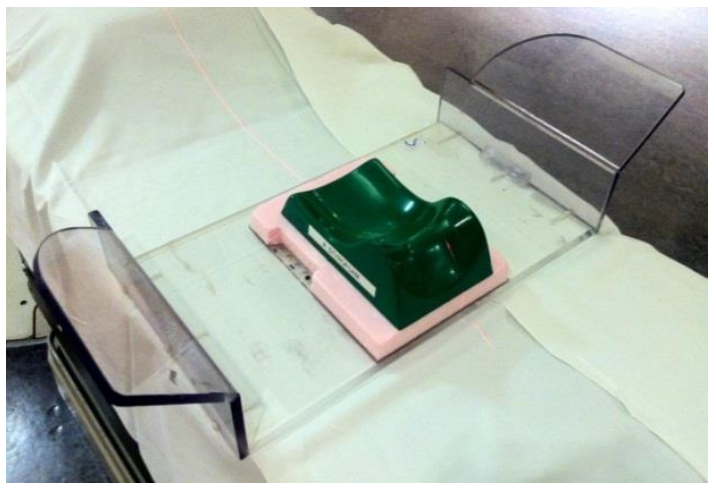

(a)

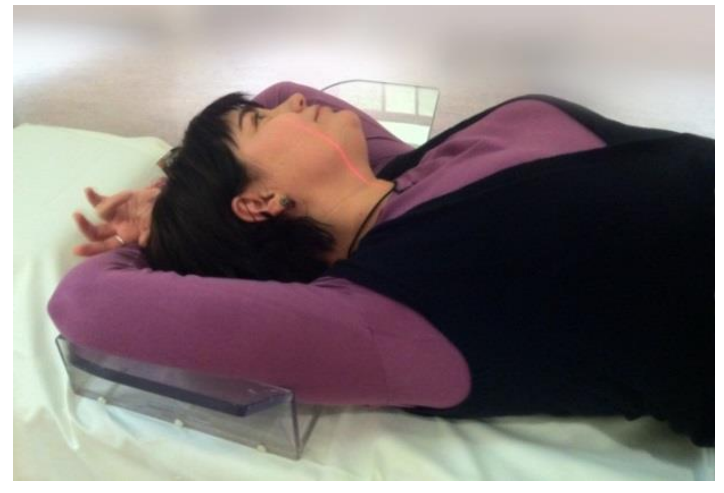

(b)

Figure 1. (a) Butterfly arm support board with head-neck support; (b) Example of patient positioning.

Both the orthogonal x-ray images and the CT data were imported into the brachytherapy planning system BrachyVision (Varian, Palo Alto, CA). The catheter positions were digitized independently using both data sets at the positions of the markers on the marker wire. The orthogonal x-ray images were processed and registered to the CT data using the registration algorithm. The resulting translations and rotations were applied to the catheter marker coordinates from the orthogonal pair reconstruction, positioning them in the CT data. The transformed catheter marker coordinates were then compared with the positions which were directly digitized from the CT data. The target registration error (TRE) ${ }^{31}$ was obtained by computing the distances between the fused and the true catheter marker positions. The marker positions within treatment volume were incorporated into the TRE calculation only.

\section{Results and Discussion}

The algorithm was evaluated using data from six patients with obstructive lung and esophagus cancer. The mean age of the patients was 65 years (range 40-82). The patients underwent the standard HDR brachytherapy treatment procedure with one treatment catheter for esophagus patients and two catheters for lung patients. 


\subsection{Algorithm Accuracy and Precision}

The results of the registration accuracy evaluation for esophagus and lung patients are summarized in table 1 .

\begin{tabular}{l|cccc|cccc|c}
\hline \multirow{2}{*}{ Patient } & \multicolumn{3}{|c|}{ TRE (Mean) [mm] } & \multicolumn{3}{c|}{ TRE (Max) [mm] } & \multirow{2}{*}{$\mathrm{N}$} \\
\cline { 2 - 7 } & $\mathrm{X}$ & $\mathrm{y}$ & $\mathrm{z}$ & Total & $\mathrm{x}$ & $\mathrm{y}$ & $\mathrm{z}$ & Total & \\
\hline Esophagus 1 & 0.8 & 1.4 & 1.4 & 2.1 & 1.8 & 1.9 & 2.6 & 3.1 & 8 \\
Esophagus 2 & 1.9 & 1.8 & 2.1 & 3.4 & 3.1 & 2.2 & 4.4 & 4.5 & 9 \\
Esophagus 3 & 1.0 & 1.3 & 1.5 & 2.2 & 2.4 & 2.8 & 2.8 & 3.7 & 9 \\
Esophagus 4 & 1.2 & 1.4 & 1.8 & 2.6 & 2.0 & 2.7 & 3.8 & 4.4 & 8 \\
Lung 1 & 0.6 & 2.3 & 3.4 & 4.1 & 0.7 & 3.2 & 5.0 & 5.7 & 10 \\
Lung 2 & 2.0 & 2.7 & 3.2 & 4.6 & 2.9 & 3.6 & 4.2 & 6.1 & 9 \\
\hline TOTAL & 1.3 & 1.8 & 2.2 & 3.2 & 3.1 & 3.6 & 5.0 & 6.1 & \\
\hline
\end{tabular}

Table 1. Registration accuracy for four esophagus and two lung cancer patients. $\mathrm{N}$ is a total number of marker positions used to compute TRE for a given patient; $\mathrm{x}, \mathrm{y}$ and $\mathrm{z}$ represent lateral, posterior-anterior and superior-inferior directions respectively; total values represent mean and maximum TREs across all marker positions for a given patient.

The mean and maximum registration errors were $3.2 \mathrm{~mm}$ and $6.1 \mathrm{~mm}$, respectively. An example of catheter reconstruction in the CT volume obtained for Lung Patient \#2 is shown in figure 2. 


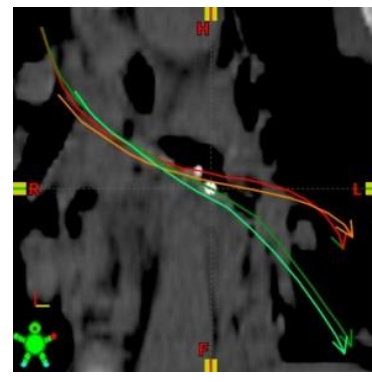

(a)

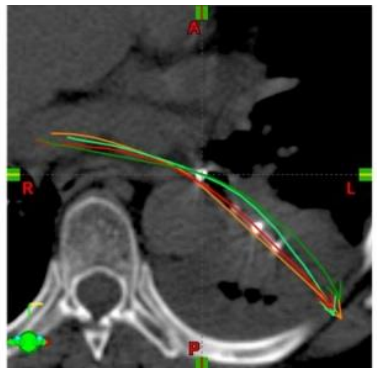

(b)

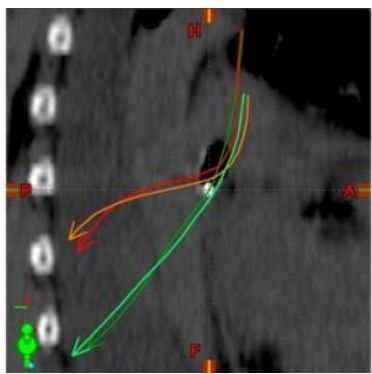

(c)

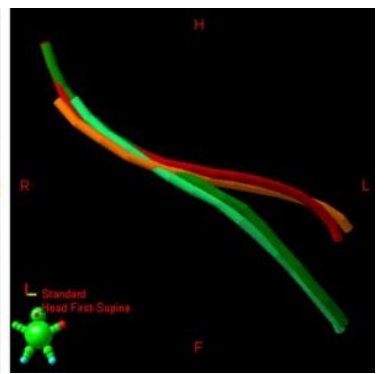

(d)

Figure 2. CT images from Lung Patient \#2 showing the true and registration-derived catheter positions. Dark green and light green represent the true and reconstructed positions of the first catheter, respectively. Red and orange represent the true and reconstructed positions of the second catheter, respectively. (a) Coronal plane; (b) Transverse plane; (c) Sagittal plane; (d) 3D rendering.

The reproducibility of the convergence of the algorithm was also evaluated. A registration solution was obtained by running the algorithm normally. The CT image was then translated and rotated by a known amount and the resulting image re-registered with the orthogonal data. For each patient this was repeated ten times varying the induced misregistration within $\pm 15^{\circ}$ and $\pm 50 \mathrm{~mm}$ for rotations and translations, respectively. For each trial, TRE averaged across all marker positions was computed. The maximum standard deviation $\sigma_{\max }$ of these TREs for each patient are shown in table 2.

\begin{tabular}{l|c}
\hline Patient & $\sigma_{\max }[\mathrm{mm}]$ \\
\hline Esophagus 1 & 0.7 \\
Esophagus 2 & 0.7 \\
Esophagus 3 & 0.7 \\
Esophagus 4 & 0.7 \\
Lung 1 & 0.9 \\
Lung 2 & 0.9 \\
\hline TOTAL & 0.8 \\
\hline
\end{tabular}

Table 2. Algorithm precision. TOTAL value represents $\sigma_{\max }$ averaged across all patients. 
The algorithm converged to a reasonable solution with maximum TRE not exceeding 6.1 $\mathrm{mm}$ in all ten trials for all six patients indicating algorithm robustness to initial displacement. It is feasible to ensure patient positioning between the c-arm and CT imaging is within $15^{\circ}$ and $50 \mathrm{~mm}$. Therefore, the registration error can be said to be 3.2 (mean) $\pm 0.8 \mathrm{~mm}$.

\subsection{Catheter Digitization Error}

The data in table 1 indicate that the error was largest in the axial direction. This was likely caused by the residual misalignment of the orthogonal images with respect to each other. During the catheter digitization process, it was discovered that the reconstructed positions of the markers relative to the bony anatomy differed considerably for posterior and lateral images. This axial mismatch, $\Delta z$, varied between $1 \mathrm{~mm}$ and $7 \mathrm{~mm}$, depending on the patient and individual marker location. The difference was caused by craniocaudal motion of the diaphragm as the patient breathed between acquisitions of the x-ray images. The reconstructed $z$-coordinate of the marker was estimated as the average of the $z$ coordinates from the two images. The true position can be anywhere between the $z$ coordinates reconstructed from individual x-ray images. As a result, the catheter marker positions are digitized with an average error of $1.7 \mathrm{~mm}(\operatorname{mean}(\Delta z) / 2)$ and maximum error of $3.5 \mathrm{~mm}(\max (\Delta z) / 2)$ in the axial direction. An example of $x$-ray data mismatch for Esophagus Patient 2 is shown in figure 3. 


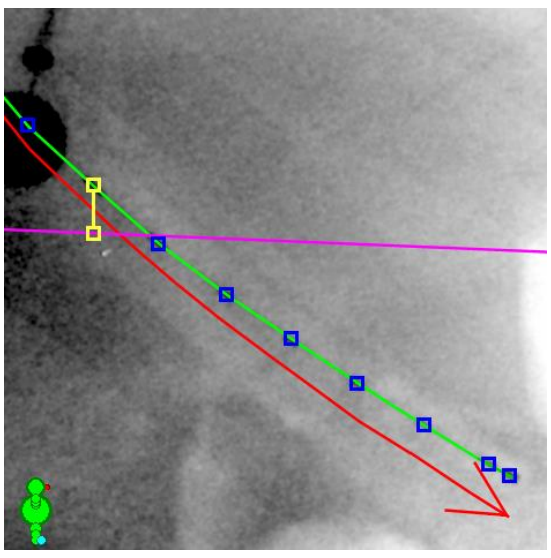

(a)

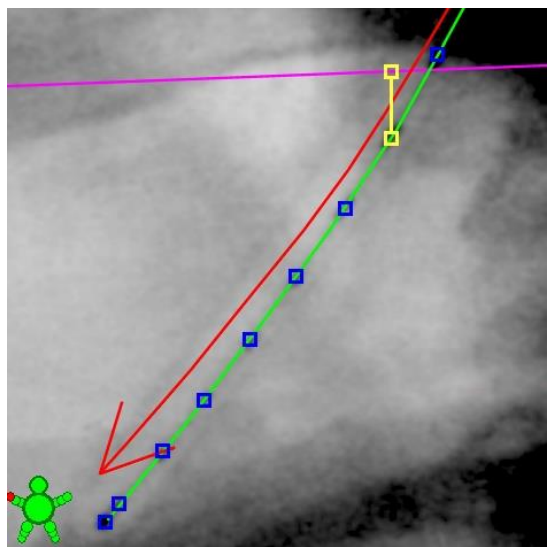

(b)

Figure 3. Example of x-ray data mismatch in the axial direction for Esophagus Patient 2. The green line shows catheter positions digitized from the current image, purple indicates the projected axial position for marker \#8 derived from the orthogonal image, dark red indicates the reconstructed catheter positions averaged over both images, and yellow indicates an axial mismatch of $5.5 \mathrm{~mm}$ between lateral and posterior images for marker \#8. (a) Lateral image; (b) Posterior image.

The x-ray data mismatch in the axial direction indicates the magnitude of catheter motion due to patient breathing between image acquisitions. It is strongly correlated with the axial component of the TRE and affects overall registration accuracy. The measured average and maximum axial mismatch for each patient along with the corresponding TREs is shown in table 3. These results show that for the majority of the patients, higher orthogonal mismatch leads to higher axial TREs.

Another major contribution to TRE was the error due to manual catheter digitization. In our previous study it was shown that the majority of the digitization error came from the axial direction due to the lower resolution of CT data in that direction. For the CT volume with a $3 \mathrm{~mm}$ slice thickness, the average and maximum digitization errors in the axial direction were $1.4 \mathrm{~mm}$ and $3.9 \mathrm{~mm}$ respectively. When we combine these errors with errors due to patient motion using summation in quadrature, we obtain $2.2 \mathrm{~mm}$ and 5.2 $\mathrm{mm}$ for the average and maximum axial error, respectively. 


\begin{tabular}{l|cc|cc}
\hline \multirow{2}{*}{ Patient } & \multicolumn{2}{|c|}{$\Delta \mathrm{z}[\mathrm{mm}]$} & \multicolumn{2}{c}{$\mathrm{TRE}_{\mathrm{Z}}[\mathrm{mm}]$} \\
\cline { 2 - 5 } & Mean & Max & Mean & Max \\
\hline Lung 1 & 4.3 & 7.0 & 3.4 & 5.0 \\
Lung 2 & 4.1 & 6.7 & 3.2 & 4.2 \\
Esophagus 2 & 3.9 & 5.5 & 2.1 & 4.4 \\
Esophagus 4 & 2.0 & 3.1 & 1.8 & 3.8 \\
Esophagus 3 & 1.9 & 2.8 & 1.5 & 2.8 \\
Esophagus 1 & 2.9 & 3.7 & 1.4 & 2.6 \\
\hline TOTAL & 3.4 & 7.0 & 2.2 & 5.0 \\
\hline
\end{tabular}

Table 3. Orthogonal data axial mismatch for each patient and resulting axial TREs. TOTAL values represent mean and maximum TREs across all patients.

Motion in the anterior/posterior (y) direction was the second largest contribution to the TRE in table 1. Although catheter motion is smaller in the transverse plane (x-y) compared to the supine direction (z), it is significant and contributes to the registration error.

The inter-operator manual digitization error for the transverse plane was estimated in our previous study. The average error for the $x$ and $y$ directions was $0.6 \mathrm{~mm}$. The maximum error was $1.3 \mathrm{~mm}$ and $0.9 \mathrm{~mm}$ for the $x$ and $y$ directions, respectively. These errors combined with those estimated for the axial direction above using summation in quadrature yield $2.4 \mathrm{~mm}$ and $5.4 \mathrm{~mm}$ for the average and maximum TRE, respectively. These values represent the registration accuracy limit for the available patient data. The TREs reported in table 1 are comparable with the above estimates. The additional sources of error that contribute to TRE include anatomical deformations between x-ray and CT data, transverse catheter motion between x-ray image acquisition, and the intrinsic registration error of the algorithm.

\subsection{Geometry Correction}

Prior to digitizing the catheters from the x-ray images a homologous point can be chosen in both views to readjust the image geometry. Normally, a marker in the treatment 
volume that is closest to the centre of the images is chosen. This corrects the axial mismatch in the vicinity of the registration point (treatment volume) and simplifies the digitization process. As a part of this study, we investigated if the incorporation of the modified registration point into the algorithm would reduce the error due to patient motion between lateral and posterior image acquisition. The DRR geometry was readjusted using the coordinates of the new registration point. However, the registration point readjustment did not result in improved registration accuracy. To the contrary, the error increased by a small percentage for five of the six patients.

\subsection{Registration Using Oblique Images}

To improve registration accuracy, one of the oblique $x$-ray images acquired at $30^{\circ}$ and $45^{\circ}$ was incorporated into the registration. The $45^{\circ}$ images were used preferentially as they balanced data between the lateral and posterior/anterior direction. The $30^{\circ}$ image was biased toward the posterior/anterior direction and was used only when the treatment table interfered with much of the beam for the $45^{\circ}$ image, which was the case for two patients (one lung and one esophagus). The similarity metric was calculated separately for the oblique image and then combined with the similarity metric for the posterior and lateral images by multiplication. Introducing the oblique image decreased the number of iterations required for optimization, suggesting that the algorithm robustness improved. The average and maximum TRE decreased by approximately $1 \mathrm{~mm}$ for Lung Patient \#1. No statistically significant improvement in registration accuracy for the other patients was detected probably due to the dominance of the reconstruction errors discussed above.

\section{Conclusions}

A 2D/3D registration algorithm for thoracic HDR brachytherapy was evaluated in a clinical setting using data from the treatments of four esophagus and two lung patients. The algorithm converged to a reasonable solution for all six patients resulting in a zero failure rate. The mean and maximum registration error was $3.2 \mathrm{~mm}$ and $6.1 \mathrm{~mm}$, 
respectively. This level of accuracy may be acceptable considering the logistical difficulties associated with the palliative brachytherapy scenario. The main contribution to registration error came from the axial direction, which is explained by the lower resolution of CT data in that direction and by the respiratory motion of the diaphragm between c-arm acquisitions. The precision of the algorithm was also evaluated and the maximum standard deviation was found to be $0.8 \mathrm{~mm}$. The target registration error (TRE) is comparable to the estimated accuracy limit that includes errors from catheter digitization and patient motion between $\mathrm{x}$-ray image acquisitions. The incorporation of oblique images into the registration likely improved the robustness of the algorithm but did not significantly improve accuracy.

Future improvements of the algorithm should focus on the two major sources of error, digitization error and patient motion. Automated marker localization in both the 2D and $3 \mathrm{D}$ data sets should reduce the inter and intra-operator digitization variation. Accounting for respiratory motion is more problematic. We recognize that the general solution of the thorax registration problem can only be achieved using non-rigid methods. However, in our case, patient anatomy deforms between orthogonal $x$-ray image acquisitions and also the CT data collection. This significantly reduces the correlation between the $2 \mathrm{D}$ images and the $3 \mathrm{D}$ volume to be registered. In addition, the low soft-tissue contrast in the $2 \mathrm{D}$ images may make deformable registration impossible, while extrapolating bony anatomy deformations to the rest of the thorax may not be sufficiently accurate. In fact, the shortage of information in the available data makes it impossible to explicitly identify the large number of degrees of freedom necessary in non-rigid registration without additional human interaction. Thus, we use a rigid transformation method, benefiting from the improved robustness. Ultimately, in order to account for patient motion, true gains in precision may require gating or synchronizing image collection within the respiratory cycle using a 4D CT technique. 


\section{ACKNOWLEDGEMENT}

This work was supported by Varian Grant \#07-583.

\section{References}

1. Celebioglu B, Gurkan OU, Erdogan S, et al. High dose rate endobronchial brachytherapy effectively palliates symptoms due to inoperable lung cancer. Jpn $J$ Clin Oncol 2002; 32:443-448.

2. Kim RY, Shen S, Duan J, Image-based three-dimensional treatment planning of intracavitary brachytherapy for cancer of the cervix: dose-volume histograms of the bladder, rectum, sigmoid colon, and small bowel. Brachytherapy. 2007; 6:187-194.

3. Onal C, Arslan G, Topkan E, et al. Comparison of conventional and CT-based planning for intracavitary brachytherapy for cervical cancer: target volume coverage and organs at risk doses. J Exp Clin. Cancer Res 2009; 28:95.

4. Lyczek J, Kazalski D, Kowalik L, et al. Comparison of the GTV coverage by PTV and isodose of $90 \%$ in 2D and 3D planning during endobronchial brachytherapy in the palliative treatment of patients with advanced lung cancer. Pilot study. J Contemp Brachytherapy. 2012; 4:113-115.

5. Kim J, Park J, 2D/3D Non-rigid Registration of Volumetric CT Lung Data using Thin-plate Spline. 7th International Workshop on Enterprise Networking and Computing in Healthcare Industry (HEALTHCOM) Busan, Korea, June, 2005; 197 201.

6. Christie NA, Pennathur A, Luketich JD, Stereotactic radiosurgery for lung tumors. In: Mould RF, Schulz RA, (Eds.), Robotic Radiosurgery, Cyberknife Society Press, Sunnyvale, CA 2005; 1:269-278.

7. Shirato H, Shimizu S, Kitamura K, et al. Four-dimensional treatment planning and fluoroscopic real-time tumor tracking radiotherapy for moving tumor. Int J Radiat Oncol Biol Phys 2000; 48:435-442.

8. Schweikard A, Shiomi H, Adler J, Respiration tracking in radiosurgery. Med Phys 2004; 31:2738-2741.

9. Markelij P, Tomaževič D, Likar B, et al. A review of 3D/2D registration methods for image-guided interventions Med Imag Anal 2010; 16:3642-3661.

10. Hamadeh A, Lavallée S, Cinquin P, Automated 3-dimensional computer tomographic and fluoroscopic image registration Comput Aided Surg 1998; 3:1453-1462. 
11. Feldmar J, Ayache N, Betting F, 3D-2D projective registration of free-form curves and surfaces. Comput Vis Image Und 1997; 65:403-424.

12. Gueziec A, Kazanzides P, Williamson B, et al. Anatomy-based registration of CTscan and intraoperative X-ray images for guiding a surgical robot. IEEE Trans Med Imaging 1998; 17:715-728.

13. Zheng G, Effective incorporating spatial information in a mutual information based 3D-2D registration of a CT volume to X-ray images. Computerized Medical Imaging and Graphics 2010; 34:553-562.

14. Russakoff DB, Intensity-based two-dimensional-three-dimensional medical image registration. Stanford University ISBN:0-496-04390-0 2004.

15. Aouadi S, Sarry L Accurate and precise 2D-3D registration based on X-ray intensity. Comput Vis Image Und 2008; 110:134-151.

16. Chen X, Varley MR, Shark LK, et al. A computationally efficient method for automatic registration of orthogonal X-ray images with volumetric CT. Phys Med Biol; 2008 53:967-983.

17. Fu DS, Kuduvalli G, A fast, accurate, and automatic 2D-3D image registration for image-guided cranial radiosurgery. Med Phys 2008; 35:2180-2194.

18. Munbodh R, Chen Z, Jaffray DA, et al. A frequency-based approach to locate common structure for 2D-3D intensity based registration of setup images in prostate radiotherapy Med Phys 2007; 34:3005-3017.

19. Jans HS, Syme AM, Rathee S, et al. 3D interfractional patient position verification using 2D-3D registration of orthogonal images Med Phys 2006; 33:1420-1439.

20. Remeijer P, Geerlof E, Ploeger L, et al. 3-D portal image analysis in clinical practice: an evaluation of 2-D and 3-D analysis techniques as applied to 30 prostate cancer patients. Int J Radiat Oncol Biol Phys 2000; 46:1281-1290.

21. Ho AK, Fu D, Cotrutz C. et al. A study of the accuracy of cyberknife spinal radiosurgery using skeletal structure tracking. Neurosurgery 2007; 60:147-156.

22. Rohlfing T, Denzler J, Grassl C et al. Markerless real-time 3-D target region tracking by motion backprojection from projection images. IEEE Trans Med Imag 2005; 24:455-1468.

23. Zhang X, Zheng GY, Langlotz F, et al. Assessment of spline-based 2D-3D registration for image-guided spine surgery Minim Invasive Ther Allied Technol 2006; 15:193-199. 
24. Hurvitz A, Joskowicz L, Registration of a CT-like atlas to fluoroscopic X-ray images using intensity correspondences. Int. J. CARS 2008; 3:493-504.

25. Dennis DA, Mahfouz MR, Komistek RD, et al. In vivo determination of normal and anterior cruciate ligament-deficient knee kinematics. J Biomech 2005; 38:241-253.

26. Jaramaz B, Eckman K, 2D/3D registration for measurement of implant alignment after total hip replacement MICCAI, LNCS 2006; 4191:653-661.

27. Penney GP, Edwards PJ, Hipwell JH, et al. Postoperative calculation of acetabular cup position using 2D-3D registration. IEEE Trans Biomed Eng 2007; 54:13421348.

28. Birkfellner W, Figl M, Kettenbach J, et al. Rigid 2D/3D slice-to-volume registration and its application on fluoroscopic CT images. Med Phys 2007; 34:246.

29. Künzler T, Registration of DRRs and portal images for verification of stereotactic body radiotherapy: a feasibility study in lung cancer treatment. Phys Med Biol 2007; $52: 2157$.

30. Zvonarev PS, Farrell TJ, Hunter R, et al. 2D/3D registration algorithm for lung brachytherapy. Med Phys 2013;

31. Kraats VD, Penney GP, Tomazevic D, et al. Standardized evaluation methodology for 2D/3D registration. IEEE Trans Med Imaging 2005; 24:1177-1189. 


\section{CHAPTER IV}

\section{PAPER III: Automated algorithm for identification and removal of external objects from patient CT scans with applications in radiation therapy and radiology}

The third paper proposed an image processing algorithm for the automatic elimination of external objects from CT images in order to improve the quality of digitally reconstructed radiographs. The algorithm employs methods of mathematical morphology with automatic threshold selection at the segmentation step. The algorithm was modified to extract patient circumference and correct for irregularities and missing data in the processed images. The application of the algorithm in both radiation therapy and diagnostic radiology scenarios was demonstrated.

The proposed algorithm was developed by P. Zvonarev, with suggestions from and under the supervision of Dr. Farrell and Dr. Wierzbicki. The evaluation of the algorithm was performed by P. Zvonarev. The patient data was collected by Dr. Sur, Emilia Timotin, and Kelly Ainsworth. The diagnostic radiology study was performed by Kelly Ainsworth under supervision of Dr. Haider. The manuscript was written by P. Zvonarev and Dr. Farrell. The manuscript was edited by Dr. Wierzbicki, Dr. Hunter, and Dr. Hayward. The manuscript was altered from its original form to comply with the style of the thesis. 


\subsection{Paper Contents}

\section{Automated algorithm for identification and removal of external objects from patient CT scans with applications in radiation therapy and radiology}

P. S. Zvonarev, K. E. Donkers Ainsworth, T. J. Farrell, R. Hunter, M. Wierzbicki, J. E. Hayward and E. Haider

Juravinski Cancer Centre and McMaster University, 699 Concession Street, Hamilton, Ontario, L8V 5C2, Canada 


\section{Abstract}

Purpose: An image processing algorithm is proposed for automated identification and elimination of external objects in CT images with applications to 3D-2D registration for lung brachytherapy, external beam treatment planning for breast treatments and automated girth calculation.

Methods: The proposed image processing algorithm utilizes the methods of mathematical morphology with automatic threshold selection at the segmentation step to eliminate external objects from CT images. The algorithm identifies and contours low and high $\mathrm{Z}$ fiducial markers used in external beam treatment planning. It also extracts the circumference of the patient and extrapolates missing sections of circumference extending outside the imaged area. The success of the algorithm was assessed by visually comparing the filtered and original CT images on a slice by slice basis.

Results: External objects were successfully eliminated from 10 CT images used for thoracic brachytherapy. The algorithm also contoured and removed external fiducial markers from three CT images used for treatment planning of breast radiotherapy. Finally, the algorithm accurately extracted the patient surface and subsequently computed girth size for 108 patients in a diagnostic radiology clinical trial setting.

Conclusions: An image processing algorithm which effectively identifies and removes external objects from CT data was developed. Applications of the algorithm in diagnostic radiology and radiation therapy were demonstrated.

\section{Introduction}

The role of the computed tomography (CT) scanner in diagnostic radiology and radiation therapy is well established and the CT data are fundamentally useful and necessary for diagnosis or treatment planning. Depending upon the patient, the CT images will contain external objects such as the imaging table, clothing, and possibly objects such as ECG 
leads and wires. In images obtained for radiation therapy planning external fiducial markers, which are used for target volume delineation and patient setup, are routinely placed during the CT data acquisition but not during treatment. These are often composed of high atomic number $(\mathrm{Z})$ materials and are generically referred to as solder markers.

While the presence of external objects is generally not a concern for diagnostic imaging, there may be some applications in which they are problematic, for example when accurate determination of the skin surface is required. In radiation therapy such external objects are more problematic. The presence of high $\mathrm{Z}$ fiducial markers can affect the external beam radiation therapy dosimetry. As a consequence, they are often manually contoured and over-ridden with a physical density corresponding to air since they are not present during treatment. In addition, the external objects can obscure patient anatomy in digitally reconstructed radiographs (DRRs). The DRRs are generated from CT images and play a crucial role in the verification of the radiotherapy treatments. Primarily, they are compared to simulator or portal $\mathrm{x}$-ray images to detect and correct patient setup errors prior to treatment. However, DRRs can also be used in $2 \mathrm{D} / 3 \mathrm{D}$ registration of pretreatment CT images with treatment $\mathrm{x}$-ray data to assist in treatment planning and delivery ${ }^{1-5}$. Hence, the presence of these external objects in the DRRs may complicate the comparison with x-ray images. This may negatively affect the quality of both manual and automatic registration of CT data with $\mathrm{x}$-ray images.

Thus, for some diagnostic applications, and for most radiation therapy applications it is important to be able to remove certain objects from CT images. A single CT data set may contain tens or even hundreds of axial images, therefore, manual image filtering would be very time consuming and thus, impractical. In this article we propose an image processing algorithm for automatic detection of external objects and their elimination from CT images. 
In order to remove the external objects surrounding the patient, automated and accurate detection of the patient's skin surface is required. When patient surface is properly determined, all objects outside the surface can be eliminated by resetting their pixel values to air equivalent. While a large number of algorithms for automated organ segmentation have been reported ${ }^{6-11}$, these algorithms ignore the presence of external objects at skin-air interface. These objects can distort the detected skin contour and prevent them from complete elimination. To guarantee complete elimination of the objects near skin surface, they have to be detected and individually processed. A few algorithms for automatic recognition and localization of fiducial markers from CT images have been reported ${ }^{12-15}$. Each of these algorithms is optimized for certain application and certain fiducial marker with known characteristics, such as geometrical shape, size, and Z value. Our goal is to identify and eliminate all external objects including high $\mathrm{Z}$ wire segments, ECG leads, and both high and low Z fiducial markers. These objects have different size, shape and $\mathrm{Z}$ values, therefore, none of the above algorithms are able to segment all of them.

The proposed image processing algorithm is developed to overcome these challenges and is based on methods of mathematical morphology (MM) ${ }^{16-18}$ with automatic threshold selection at the segmentation step. We further developed the algorithm to extract patient circumference and correct for irregularities and missing data in the processed images. Applications of the algorithm in both radiation therapy and diagnostic radiology scenarios are demonstrated.

\subsection{Radiation Therapy}

\subsubsection{Image Guidance for Lung Brachytherapy}

High dose rate (HDR) brachytherapy is performed by inserting hollow treatment catheters into the lung under bronchoscopic guidance and delivering treatment by remotely sending a radioactive source into the treatment volume through the catheters. Insertion and 
treatment are performed in a shielded suite. Treatment planning is typically performed using an orthogonal pair of planar X-ray images obtained in the treatment suite. While it would be beneficial to have CT data available for treatment planning, it is impractical to move the patient from the procedure room to the CT suite because they are under sedation, connected to ECG monitors, have multiple catheters inserted, and are connected to oxygen. However, it is possible to register the 2D orthogonal X-ray images with a 3D CT image obtained previously, usually for the planning of the external beam phase of therapy ${ }^{1}$. This allows virtual treatment catheters to be digitized in the CT data to facilitate treatment planning. The presence of ECG leads and other external objects can obscure the anatomical information necessary to accurately register the 2D and 3D data.

\subsubsection{External Beam Radiotherapy of Breast Cancer}

External beam radiation therapy planning for breast treatment is well described elsewhere ${ }^{19-22}$. For these patients, two different types of external fiducial markers are typically applied at the time of CT scanning. The first are small solder markers that aid in reproducibly setting the patient up for daily treatment. The second is a long hollow tube that is applied to the skin to define the external breast. The tube is used for defining the extent of the external beam treatment volume. The presence of these markers affects the accuracy of the dose calculation since they are not placed on the patient during treatment. Thus, both types of markers are manually contoured on every CT slice and the physical density is set to air.

The algorithm was applied to patient data from both of these scenarios to remove the external objects from the CT data. This was used to determine the accuracy and utility of the algorithm in the radiation therapy setting. 


\subsection{Diagnostic Radiology}

In this scenario we used the algorithm to address a research question in diagnostic radiology, and the application was geared towards designing imaging protocols rather than for individual patient studies.

Appendicitis is the most common cause of acute abdominal pain requiring surgery ${ }^{23}$. Early diagnosis and treatment are crucial to avoid complications such as perforation. CT and ultrasound (US) are widely recognized as useful tools for diagnosing acute appendicitis. The conventional protocol utilizes intravenous (IV) and oral contrast ${ }^{24,25}$; although, more recent work focuses on low-dose CT techniques and comparing unenhanced versus enhanced (IV, oral, or both) protocols $(26,27)$. Peck et al. list the advantages of not using contrast, which includes ( $i$ ) not obscuring small appendicoliths, (ii) ingestion of oral contrast may be unpleasant for patients or unachievable for nauseated patients, (iii) patients may develop allergic reactions or acute renal failure with IV contrast (iv) and contrast slightly increases the cost of the study ${ }^{28}$. Another important issue, particularly for oral contrast, is the 1-2 hour time delay between the study request, oral contrast administration and acquiring the $\mathrm{CT}$, resulting in further diagnostic delay.

As such, another well-studied protocol is unenhanced CT without the use of oral or IV contrast $^{28-36}$ in both pediatric ${ }^{28,30,33,35}$ and adult populations where the reported sensitivity and specificity of diagnosing appendicitis ranges from 87-96\% and 91-98\% respectively ${ }^{31,32,35}$. With non-contrast $\mathrm{CT}$, the patient's intra-abdominal fat provides enough "natural" contrast to visualize the appendix by accentuating periappendiceal inflammatory change, which facilitates the diagnosis of acute appendicitis. Unenhanced scans are also useful for identifying alternate diagnoses including diverticulitis, urinary tract disease, adnexal pathology, and small bowel disease ${ }^{32}$. While these earlier studies conclude that unenhanced CT is accurate in diagnosing appendicitis, it is acknowledged that ideal patient for this protocol are patients with a normal or obese body habitus ${ }^{33,35}$ and that for slender patients with little periappendiceal fat, the diagnosis of appendicitis is 
difficult $^{31,32,38}$. Previous studies have shown statistically significant correlation between visibility of the appendix and degree of pericecal fat $^{39,40}$. While unenhanced CT is a useful tool in select patients, particularly as it relates to improving delays in diagnosis, the question becomes: is it possible to determine which patients have enough periappendiceal fat to be a suitable candidate for an unenhanced CT? To our knowledge, this remains unanswered in the literature with only one study reporting on abdominal circumference as a secondary question previously ${ }^{41}$. The objective of the clinical trial was to determine if there is an abdominal girth size above which unenhanced CT scanning is adequate in identifying a normal appendix.

Girth may be derived from axial slices of previously acquired CT images but additional surface objects could be problematic in accurately defining the skin surface. Also, parts of the body may extend beyond the CT field of view, making direct calculation of the girth size impossible. The proposed algorithm cleans the surface of the patient from additional objects. It has also been modified to automatically detect cases where the patient exceeds the field of view and estimates girth by extrapolating missing sections of the circumference. This algorithm was employed in a clinical study in which the girth was calculated retrospectively from unenhanced helical CT scans for a large number of patients.

\section{Methods}

\subsection{External Objects Filtering}

The algorithm is a sequence of MM filters, which produce the series of images shown in figure 1. Image $I_{x}$ corresponds to figure 1(x) for $x=a \rightarrow b$. To distinguish pixels belonging to external objects from those of the patient the original grayscale 4096-level CT image, $I_{a}$, was first processed by the threshold filter resulting in binary image $I_{b}$. The correct choice of threshold level $\theta$ is critical for proper image segmentation, the goal of which is 
to eliminate as many pixels as possible that belong to background and surrounding objects without compromising the continuous edge of the patient body. The intensity histogram $h_{a}(i)$ for a typical CT image is shown in figure 2 and has two distinct areas with high probabilities: a low intensity area corresponding to background and objects with low $\mathrm{HU}$, and a mid $\mathrm{HU}$ area corresponding to patient soft tissues. It was found experimentally that optimal threshold level value $\theta$ ranged between $\theta_{\min }=-180$ and $\theta_{\max }=-50 \mathrm{HU}$ depending on the specific CT data set. Thus, the search range for optimal $\theta$ was limited between $\theta_{\min }$ and $\theta_{\max }$. The optimal threshold $\theta$ was calculated by finding the minimum of $\tilde{h}_{a}(i)$ closest to $\theta_{\max }$, such that $0<\tilde{h}_{a}(\theta) \leq 0.1 \times H$, where $\tilde{h}_{a}(i)$ is the intensity distribution function $h_{a}(i)$ smoothed by a 1-dimensional order 7 mean filter and $H$ is the maximum value of $h_{a}(i)$ for $i>\theta_{\min }$.

The next step was to eliminate voids within the body that appeared after threshold filtering in the areas with low HU values (e.g. lung, lumen, etc.). This is achieved by recursively dilating the seed image conditioned by the mask, where the seed image is the MM Laplacian of the inversed image $I_{b}$ and the mask is the inversed image $I_{b}$. This filling filter applied to image $I_{b}$ generates image $I_{c}$.

Image $I_{c}$ contains artifacts from objects with high $\mathrm{HU}$ values such as sections of the treatment table, cables, and fiducial markers. These artifacts were removed using a MM opening filter with a $7 \times 7$ disk structuring element resulting in image $I_{d}$. Finally, the borders are smoothed using a median filter with a $5 \times 5$ square window and dilated twice with a $3 \times 3$ diamond structuring element to account for low intensity pixels near the patient border yielding image $I_{e}$. Image $I_{e}$ accurately maps the pixels belonging to the patient. The intersection of image $I_{e}$ and original image $I_{a}$ results in grayscale CT image $I_{f}$ with eliminated external objects. 


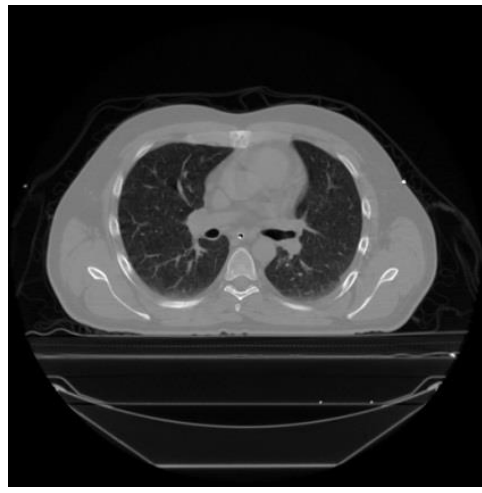

(b)

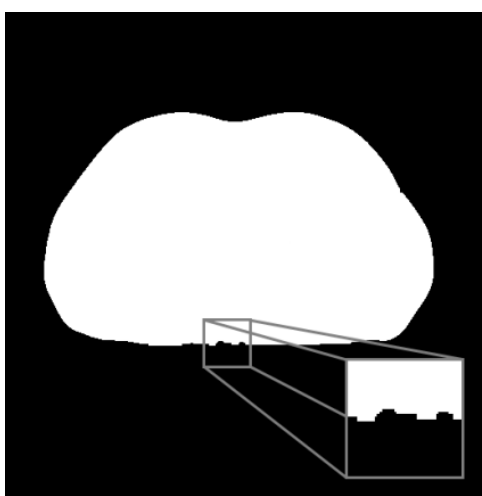

(d)

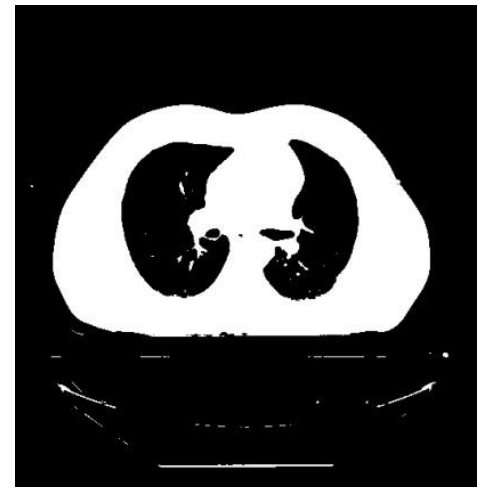

(b)

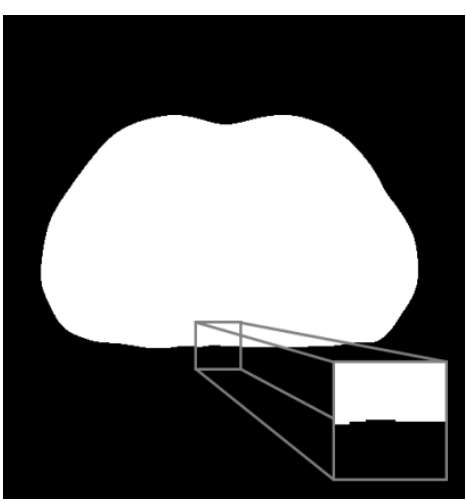

(e)

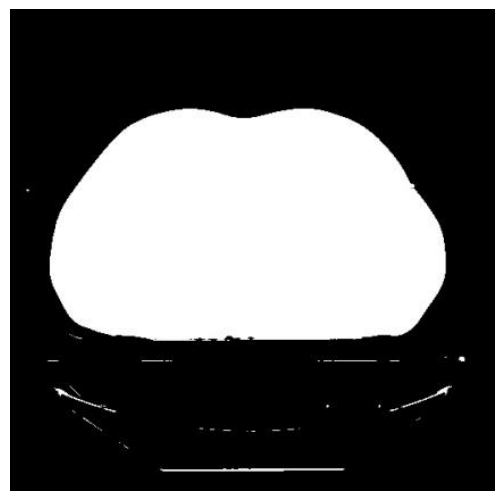

(c)

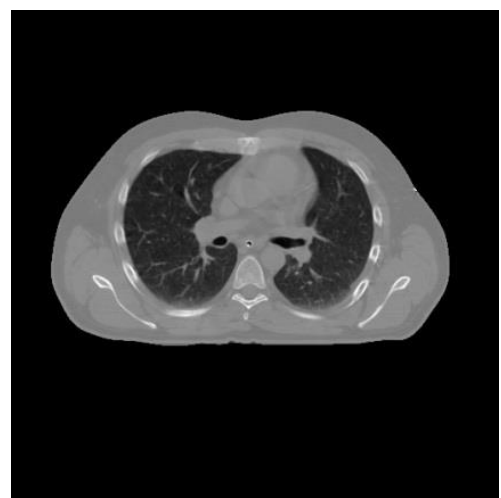

(f)

Figure 1. Typical CT image processed by subsequent application of MM filters: (a) Original CT image; (b) Threshold filter; (c) Holes filling filter; (d) Opening filter; (e) Median filter; (f) CT image with eliminated external objects.

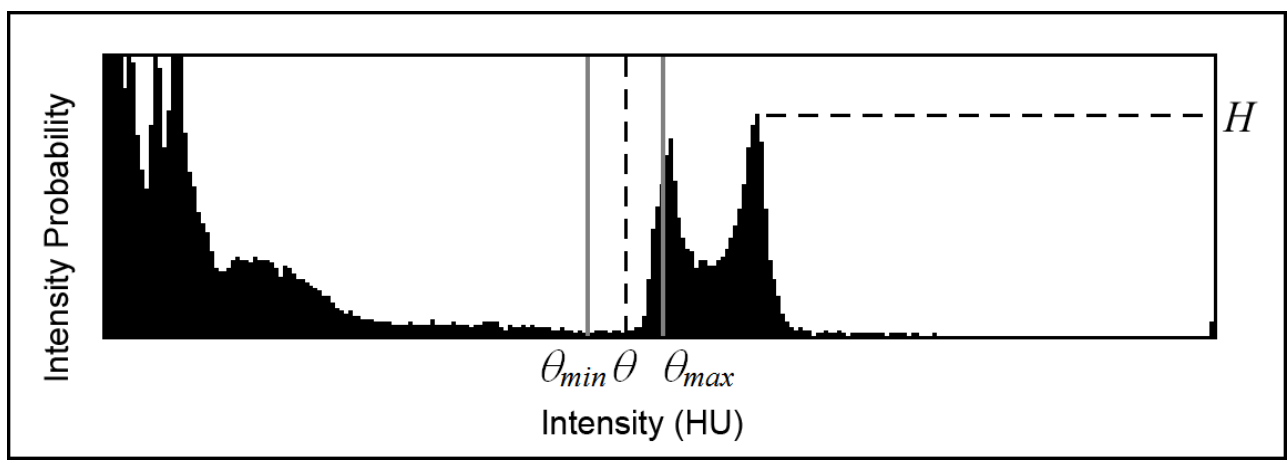

Figure 2. The intensity histogram for a typical CT image. 
The algorithm described above effectively removes the treatment table, clothing, ECG leads and most of the wires and fiducial markers surrounding the patient. However, small portions of the wires and markers may not be filtered completely from images when they are not outside the patient surface. The example of such case for brachytherapy is shown in figure 3(a). In order to remove the wires and markers completely, additional filtering is required. First, a $5 \mathrm{~mm}$ deep region at the skin is selected based on image $I_{e}$. Within this region, high intensity pixels are detected using threshold filter with level $\theta_{2}=500 \mathrm{HU}$, and dilated twice with $3 \times 3$ diamond structuring element. The detected pixels are then replaced with the mean intensity computed from a two pixel thick surrounding region. The resulting image is shown in figure $3(\mathrm{~b})$.

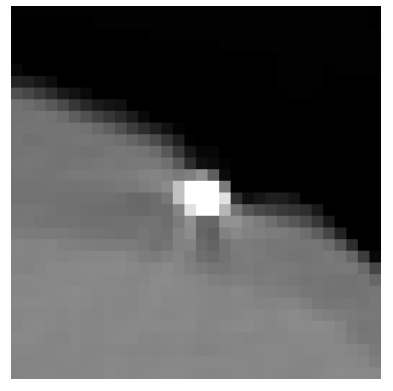

(a)

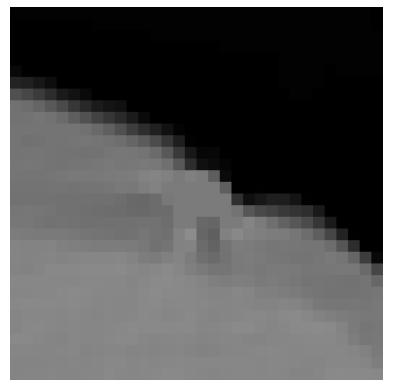

(b)

Figure 3. Example of output obtained when removing a wire from a CT image: (a) Initial result with the wire not eliminated; (b) Result obtained with additional filtering.

\subsection{Automated Marker Contouring}

In external beam radiation therapy planning for breast treatments it is useful to be able to automatically detect and contour fiducial markers. Since there are two types of markers with different average HUs, different approaches are required to segment each type of marker.

The solder marker is detected by first selecting the $10 \mathrm{~mm}$ thick region extending $5 \mathrm{~mm}$ inside and $5 \mathrm{~mm}$ outside the skin based on image $I_{e}$. Within this region, high intensity pixels are detected using a threshold filter with level $\theta=500 \mathrm{HU}$, and dilated with a $3 \times 3$ 
diamond structuring element. The Laplacian with $3 \times 3$ diamond structuring element yields a one pixel thick contour of the marker.

The plastic filament marker is detected by selecting the $5 \mathrm{~mm}$ thick region outside the skin. Within this region, pixels are detected using a threshold filter with level $\theta=-470$ HU. Due to comparable HUs of the plastic filament marker and skin at the surface, the marker appears as a set of often unconnected pixels. Thus, direct contouring of the marker is challenging. Instead, the contour can be approximated as a circumference of predefined diameter at the geometric centre of the detected pixels. The diameter of the circumference is chosen to be larger than the physical diameter of the marker to make sure the contour entirely encompasses the marker. To prevent the contour encroaching on the patient surface, the contour is restricted by $I_{e}$.

\subsection{Girth Size Calculation}

In order to compute the girth from a CT image, a one pixel thickness circumference is extracted from image $I_{e}$ using a Laplacian operator with a $3 \times 3$ diamond mask. The pixels are then vectorized into an $N$ long array of pixel coordinates $\left\{\vec{p}_{i}\right\}$ recorded in a clockwise direction. The length of circumference $L$ is then calculated by subsequent addition of the distances between adjacent pixels:

$$
L=\sum_{i=1}^{N-1}\left\|\vec{p}_{i+1}-\vec{p}_{i}\right\|+\left\|\vec{p}_{N}-\vec{p}_{1}\right\|
$$

An example of abdominal circumference extraction from a typical axial CT image is shown in figure 4. 


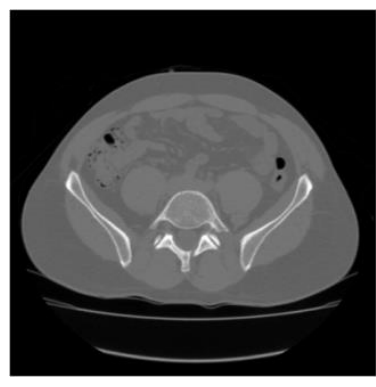

(a)

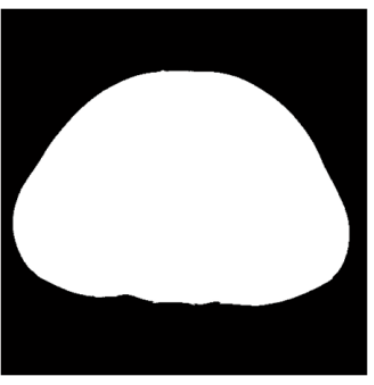

(b)

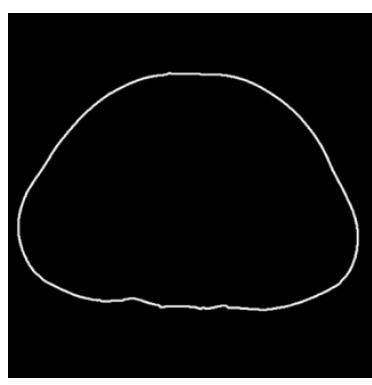

(c)

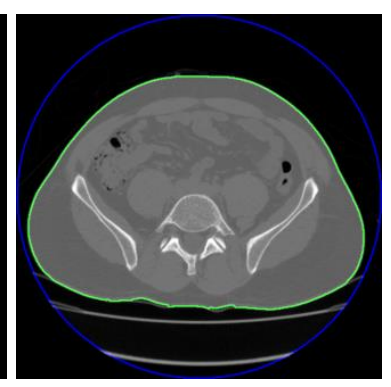

(d)

Figure 4. The sequence of images obtained using MM filters when extracting the circumference from a typical CT image: (a) Original CT image; (b) Pixels mapping the patient tissues; (c) Laplacian filter; (d) Overlay of extracted circumference with the original CT image.

In several CT images used in this study, a portion of the body did not fit entirely into the field of view making direct calculation of circumference length impossible. One such case is shown in figure 5(a). To overcome the problem, the algorithm was modified to automatically detect such cases and extrapolate missing circumference segments with a spline allowing for girth estimation.

In order to detect the portions of the body extending beyond the field of view, image $I_{e}$ is intersected with an image of a one pixel thick circumference encompassing a circular field of view resulting in image $I_{g}$ (figure 5(b)). If image $I_{g}$ is empty, no extrapolation is required, however if intersecting pixels are found, the user is warned that the circumference is going to be extrapolated, which may lead to an inaccuracy in girth estimation. The detected pixels appear as segments of a circumference in image $I_{g}$. Let $\vec{p}_{n}$ and $\vec{p}_{m}$ be the end points for the left segment in image $I_{g}$. The missing circumference segment is extrapolated using a spline with the following pixel positions serving as knots: $\vec{p}_{n-20}, \vec{p}_{n-10}, \vec{p}_{n}, \vec{p}_{m}, \vec{p}_{m+10}, \vec{p}_{m+20}$. The length of the spline segment is computed from $\vec{p}_{n}$ to $\vec{p}_{m}$ yielding the length of the missing segment. The procedure is repeated for other segments in image $I_{g}$. 


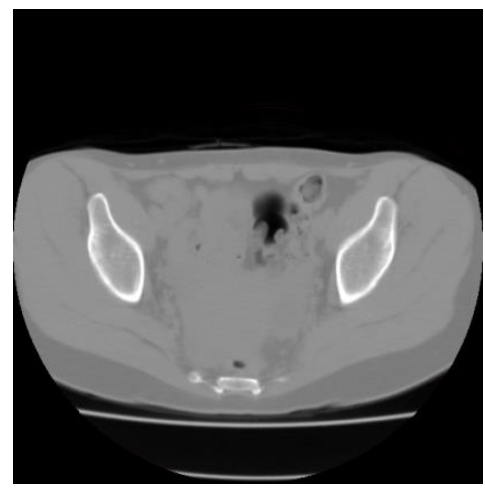

(a)

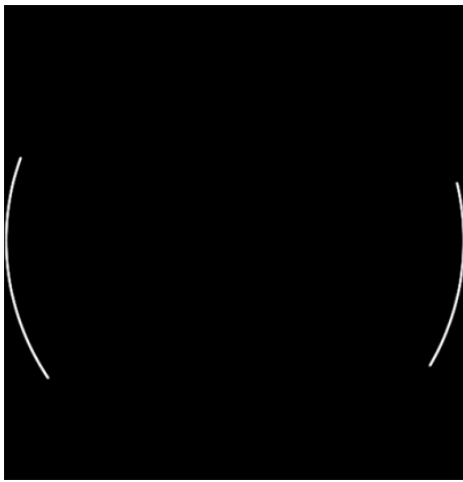

(b)

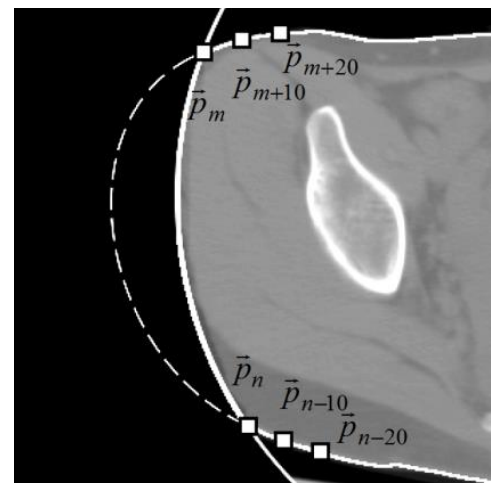

(c)

Figure 5. (a) CT image in which portions of the body extend outside the field of view; (b) Segments of circumference which require correction; (c) Example of a circumference segment extrapolated using a spline.

\subsection{Algorithm Verification}

The quality of CT filtered data was evaluated by visual inspection of each CT slice processed by the algorithm and comparing it with original image. The filtering was deemed successful if all external objects were removed by the algorithm, and if the patient body contour matched the contour in the original image. To further evaluate the quality of wire removal from processed CT data, the lateral and posterior/anterior DRRs were generated using a ray casting method. The filtering was deemed successful if no wires were visible in both DRRs.

To evaluate the accuracy of the girth predicted by the extrapolation algorithm the following method was adopted. The true girth $L$ was calculated for a CT image in which the entire body was initially within the field of view. The images were then rescaled with a scaling factor $f>1$ in such a way that approximately $25 \%$ of the abdominal circumference extended outside the field of view. The girth $L_{f}^{\prime}$ was calculated for the rescaled images, which required extrapolation of the missing $\sim 25 \%$ circumferential 
length. The estimated girth $L^{\prime}=L_{f}^{\prime} / f$, corrected for scaling, was then compared with true girth $L$, which yields the extrapolation error of the algorithm.

\subsection{Clinical Trial Design}

A retrospective review of adult and pediatric patients at our tri-site hospitals who underwent renal colic CT scans, was conducted using our electronic picture archiving and communication system (PACS). We chose to evaluate patients who underwent renal colic CT scans as it is performed without oral or IV contrast. Patients were excluded if at the time of the scan they were an in-patient, had previous abdominal surgery, had evidence of current appendiceal or cecal pathology, had the presence of an appendicolith, residual oral contrast from a previous study or if the soft tissues at the level of the iliac crest were not included in the field of view. Patients underwent contiguous axial scans through the abdomen and pelvis with either a 16- or 64-slice multi-detector CT (Toshiba, Aquilion; GE Medical Systems, LightSpeed VCT) as per a routine, standard-dose renal colic protocol with 2.0- or 2.5-mm section thickness and 2.0-mm coronal reformatted images. One radiology resident (post-graduate year 3 at the time of interpretation) and one staff abdominal radiologist independently reviewed the scans to determine if they could visualize a normal appendix. A normal appendix was defined as a thin-walled $(<6 \mathrm{~mm})$, blind-ending tubular structure originating from between the ileocecal valve and cecal apex. Readers were able to interpret the scans in both the axial and coronal planes and were free to change the window settings. For each study, the reader was asked to record whether the appendix was "seen" or "not seen." Frequency counts were used to tabulate whether a normal appendix was "seen" or "not seen" where "seen" meant a high level of confidence and "not seen" meant the reader was unsure or was not able to identify a normal appendix. A one-way analysis of variance (ANOVA) was used to determine if there was a mean difference between appendices "seen" and "not seen." A two-way ANOVA was used to determine if there were any gender differences. Receiver operating characteristic (ROC) curves were constructed to determine the abdominal girth cut-off 
points. A repeated measures ANOVA was used to calculate the inter-observer reliability co-efficient. This study was approved by our shared institutional Research Ethics Board.

\section{Results}

\subsection{External Objects Filtering for HDR Brachytherapy}

The algorithm was evaluated using CT data sets from 10 patients undergoing lung and esophagus brachytherapy. Successful elimination of external objects was achieved for all 10 data sets. The lateral DRRs generated from original and filtered CT data for a typical esophagus patient with attached ECG leads, wires, and treatment catheter are shown in figure 6. This figure demonstrates that all external wires and ECG leads were successfully removed while the treatment catheter inside esophagus remained intact.

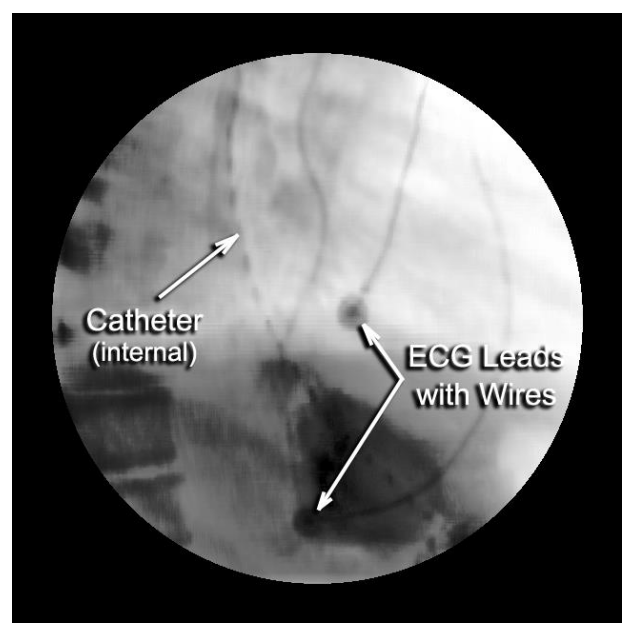

(a)

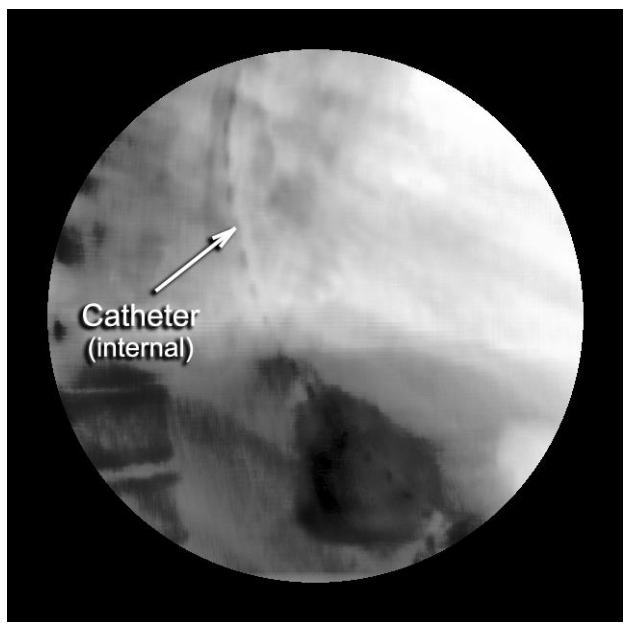

(b)

Figure 6. Example of lateral DRRs for an esophagus brachytherapy patient: (a) Generated from original CT data; (b) Generated from CT data with eliminated external objects. 


\subsection{Automated Marker Contouring and Removal for Breast External Beam Radiotherapy}

The algorithm was able to successfully identify and remove external fiducial markers placed on the skin of three patients. This allowed for automated contouring of the fiducial markers as well as removal from the CT image. An example of s successful marker contouring result is shown in figure 7 where the high $\mathrm{Z}$ solder (figure 7(a)) and the low $\mathrm{Z}$ fidudial markers are identified (figure 7(b)).

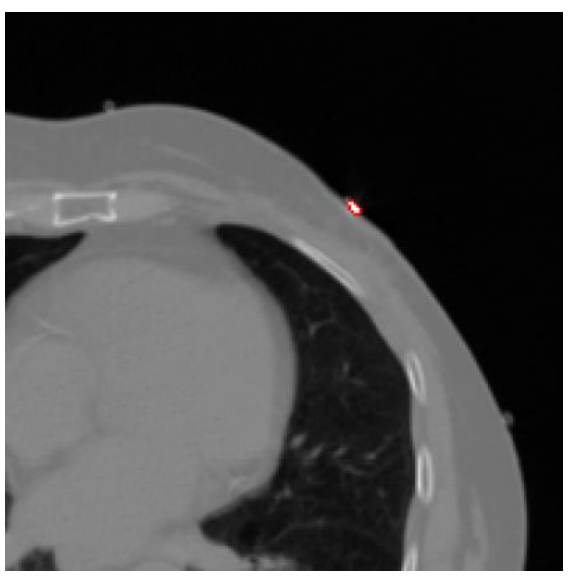

(a)

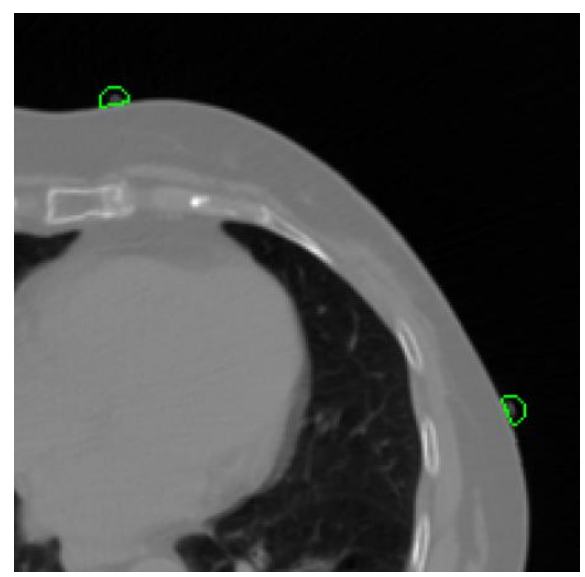

(b)

Figure 7. Example of automatic marker contouring for external beam breast radiotherapy:

(a) Solder marker; (b) Plastic filament marker.

\subsection{Girth Calculation and Appendix Visualization in Diagnostic Radiology}

The accuracy of the surface extrapolation algorithm was evaluated for ten patient data sets. The relative error between the true and the extrapolated circumference varied between $0.5 \%$ and $6 \%$. The average relative error was $3 \%$. The absolute error depended on the size of the patient and varied between $0.7 \mathrm{~cm}$ and $6 \mathrm{~cm}$. The average error was 3 $\mathrm{cm}$. 
The circumference was extracted and girth was calculated for one-hundred and eight patients at the level of the iliac crests. Visual study of the images with overlaid circumference showed a close match with the contour of the body, suggesting accurate calculation of the girth. Out of 108 patients, two patients required circumference extrapolation to estimate the girth. Since these calculations were performed retrospectively there was no true girth available for comparison.

A normal appendix was "seen" in 74 patients and "not seen" in 34 patients. The abdominal girth was (on average) $19.42 \mathrm{~cm}$ larger for those patients in whom the appendix was visible $(\mathrm{p}<0.001)$. The mean abdominal girth in the appendix "seen" patients was $121.12 \mathrm{~cm}(\mathrm{SD}=16.03 \mathrm{~cm})$ and $101.70 \mathrm{~cm}(\mathrm{SD}=20.03 \mathrm{~cm})$ in the appendix "not-seen" patients. The ability to visualize the appendix by gender was not statistically significant $(\mathrm{p}=0.09)$. Statistically significant cut-off girths were determined for the male, pediatric, adult, and combined pediatric and adult subgroups. The cut-off girth for the female subgroup was not statistically significant $(\mathrm{p}=0.058)$. Using the staff abdominal radiologist data for the combined pediatric and adult subgroup, the cut-off girth was 103 $\mathrm{cm}(\mathrm{AUC}=.651 ; 95 \%$ confidence interval, 53-77). Comparatively, using the resident radiologist data for the combined pediatric and adult subgroup, the cut-off girth was 106 $\mathrm{cm}(\mathrm{AUC}=.775 ; 95 \%$ confidence interval, 67-88).

\section{Discussion}

There are many instances in which patients will undergo a CT scan with external objects present. These may be incidental: ECG leads, blankets and clothing, or necessary: external fidicual markers for external beam radiation therapy. For most diagnostic radiology procedures their presence is irrelevant. However, when the CT data is processed using automated algorithms they can be problematic. In radiation therapy applications they can also affect the accuracy of the dose calculation. An algorithm which 
uses simple morphological operations was developed to identify and remove external objects from CT images.

The algorithm was tested on groups of thoracic HDR brachytherapy patients, breast external beam radiation therapy patients and pelvic diagnostic CT scans for the purpose of identifying the appendix without exogenous contrast agents. In all cases the algorithm was able to correctly identify the patient external surface and remove all external objects. In addition, for a small number of large patients a second algorithm was used to extrapolate the patient's surface beyond the field of view of the CT bore.

In radiation therapy the use of external fiducial markers is common, and the practice of manually contouring them, both to identify them and to set their physical density equal to air is time consuming and subject to human error. This algorithm successfully defines both high $\mathrm{Z}$ and low $\mathrm{Z}$ external markers to eliminate the need for manual contouring. In addition, this allows the removal of external fiducial markers before the creation of DRRs by the treatment planning system. This was shown to be important for a 3D-2D registration technique in HDR brachytherapy ${ }^{1}$. This may also be important for automated techniques in image guided radiation therapy (IGRT) in which images taken on the treatment unit are matched to DRRs from the planning system in order to verify patient setup prior to treatment delivery. In such instances, external objects may clutter the image and decrease the effectiveness of the image matching algorithms.

The images produced by the algorithm were further processed to calculate the patient circumference as part of a clinical trial in diagnostic radiology. The patient surface contours calculated by the algorithm closely matched the patient contour observed by a trained radiologist. A further algorithm was developed to extrapolate the patient surface beyond the CT field of view, and was shown to be accurate to $\sim 3 \%$. For example, in radiation therapy it may be useful to estimate treatment depths for extremely obese patients who are not accommodated by the standard CT simulation bore radius. 
Alternatively it may be useful in serial measurements of patients whose weight is changing over the course of radiation treatment.

Regarding the results of the clinical trial: to our knowledge, there are no studies in the literature primarily reporting a clinically measureable method for selecting patients in whom an unenhanced CT scan is adequate in visualizing the appendix. One study reports on abdominal circumference secondarily as it relates to appendix visibility ${ }^{41}$. This group reports no significant correlation between appendix visibility with abdominal dimension (diameter, circumference, cross-sectional area), nor degree of pericecal and visceral fat. However, out of the 68 patients included in that study, the abdominal circumference ranged from 73-123 cm compared to out study of 108 patients where the abdominal circumference ranged from $92-161 \mathrm{~cm}$. This difference (smaller sized patients in the previous study) may explain the failure to find a clinically significant correlation between appendix visualization and abdominal dimensions. There are several advantages to unenhanced CT scans, most importantly, avoiding the 1-2 hour diagnostic time delay necessary following oral contrast administration. In our study, patients with an abdominal girth exceeding $105 \mathrm{~cm}$ have sufficient periappendiceal fat to act as "natural" contrast to readily identify the appendix. Thus, for patients not meeting this girth-size criteria, an ultrasound or CT scan with contrast should remain primary imaging choices; this would primarily apply to slender and pediatric patient populations. This protocol may be appealing to busy radiology practices, particularly those providing emergency department imaging services. We chose to retrospectively evaluate the ability of unenhanced CT examinations to identify a normal, as opposed to acutely inflamed, appendix as we felt there was little point in a prospective studying, given the much greater financial and personnel resources and lack of literature on this topic. We felt that evaluating for a "normal" appendix would yield a more conservative cut-off girth as acute appendicitis, given the appendiceal and periappendiceal inflammatory change, make the inflamed appendix more conspicuous and readily apparent on a CT scan when compared to a normal appendix. Visibility by gender was not statistically significant; that is, the 
appendix was seen when patients of either gender had larger abdominal circumferences. Our study had several limitations. The retrospective nature may have introduced patient selection bias. Patients were excluded if they had abdominal pathology that would make the appendix more conspicuous, for example, inflammation surrounding an appendiceal or cecal pathology, an appendicolith or residual oral contrast. However, we felt that including such patients would underestimate the cut-off girth. Conversely, if the patient had previous abdominal surgery, correctly determining the lack of apendix in patients with prior appendectomies would overestimate the cut-off girth. We acknowledge that emergency department patients do not necessarily present in a straightforward way with a clear unifying diagnosis such as acute appendicitis. In these scenarios, complex cases certainly deserve greater consideration with respect to the appropriate CT protocol regardless of girth.

\section{Conclusions}

An image processing algorithm which effectively identifies and removes external objects from CT data was developed. Applications of the algorithm in diagnostic radiology and radiation therapy were demonstrated. The algorithm evaluation in the HDR brachytherapy application showed successful object removal for 10 patient data sets. The algorithm accurately extracted patient surface and subsequently computed girth size for 108 patients in a clinical trial in the diagnostic radiology setting. The method is simple to implement and has many potential applications for CT imaging.

\section{References}

1. Zvonarev PS, Farrell TJ, Hunter R, Wierzbicki M, Hayward JE (2013) 2D/3D registration algorithm for lung brachytherapy. Med Phys 2013

2. Künzler $\mathrm{T}$ (2007) Registration of DRRs and portal images for verification of stereotactic body radiotherapy: a feasibility study in lung cancer treatment. Phys Med Biol; 52:2157. 
3. Chen X, Varley MR, Shark LK, Shentall GS, Kirby MC (2008) A computationally efficient method for automatic registration of orthogonal X-ray images with volumetric CT. Phys Med Biol; 53:967-983

4. Jaramaz B, Eckman K (2006) 2D/3D registration for measurement of implant alignment after total hip replacement MICCAI, LNCS; 4191:653-661

5. Bastida-Jumilla MC, Larrey-Ruiz J, Verdú-Monedero R, Morales-Sánchez J, SanchoGómez JL (2011) DRR and portal image registration for automatic patient positioning in radiotherapy treatment. J Digit Imaging 24(6):999-1009

6. Zhou X, Hara T, Fujita H, Yokoyama R, Kiryu T, Hoshi H. (2004). Automated segmentations of skin, soft-tissue, and skeleton, from torso CT images. In Medical Imaging 1634-1639. International Society for Optics and Photonics

7. Soler L, Delingette H, Malandain G, Ayache N, Koehl C, Clément JM, Marescaux, J. (2000). An automatic virtual patient reconstruction from CT-scans for hepatic surgical planning. Studies in health technology and informatics. (70) 316-22.

8. Zhou X, Hara T, Fujita T, Ida Y, Katada K, Matsumoto K (2002). Extraction and recognition of the thoracic organs based on 3D CT images and its application. Proc. of the 16th International Congress and Exhibition of Computer Assisted Radiology and Surgery 776-781.

9. Kitasaka T, Mori K, Hasegawa J, Toriwaki J, Katada K (2002). A method for automated extraction of aorta and pulmonary artery using line models from 3-D chest X-ray CT images with contrast medium. Proc. of 16th Conf. on Pattern Recognition, 3:273-276.

10. Mori K, Hasegawa J, Suenaga Y, Toriwaki J (2000). Automated anatomical labeling of the bronchial branch and its application to the virtual bronchoscopy system. IEEE Trans. Med. Imag. 19:103-114.

11. Sharma N, Aggarwal LM. (2010). Automated medical image segmentation techniques. Journal of medical physics/Association of Medical Physicists of India, 35(1): 3 .

12. Wang M, Song Z (2009). Automatic localization of the center of fiducial markers in 3D CT/MRI images for image-guided neurosurgery. Pattern Recognition Letters 30(4):414-420. 
13. Papavasileiou, P, Flux GD, Flower MA, Guy MJ (2001). Automated CT marker segmentation for image registration in radionuclide therapy. Physics in Medicine and Biology 46(12):N269

14. Krishnan R, Hermann E, Wolff R, Zimmermann M, Seifert V, Raabe A. (2003). Automated fiducial marker detection for patient registration in image-guided neurosurgery. Computer Aided Surgery 8(1):17-23.

15. Zheng G, Gerber N, Widmer D, Stieger C, Caversaccio M, Nolte LP, Weber S (2010). Automated detection of fiducial screws from CT/DVT volume data for image-guided ENT surgery. In Engineering in Medicine and Biology Society (EMBC), 2010 Annual International Conference of the IEEE 2325-2328.

16. Dougherty ER, Lotufo, RA (2003) Hands-on morphological image processing. Bellingham: Spie.

17. Haralick RM, Sternberg SR, Zhuang X (1987) Image analysis using mathematical morphology. Pattern Analysis and Machine Intelligence, IEEE Transactions on; $4: 532-550$

18. Fang Z, Junpeng Z, Yulei M Medical Image Processing Based on Mathematical Morphology.

19. Njeh CF, Saunders MW, Langton CM (2012) Accelerated partial breast irradiation using external beam conformal radiation therapy: A review. Critical Reviews in Oncology / Hematology 81(1):1-20

20. Baglan KL, Sharpe MB, Jaffray D, Frazier RC, Fayad J, Kestin LL, Remouchamps V, Martinez AA, Wong J, Vicini FA (2003) Accelerated partial breast irradiation using 3D conformal radiation therapy (3D-CRT). Int J Radiat Oncol Biol Phys 55:302-311.

21. Hurkmans CW, Saarnak AE, Pieters BR, Borger JH, Bruinvis IAD (2000) An improved technique for breast cancer irradiation including the locoregional lymph nodes. Int J Radiat Oncol Biol Phys, 47(5):1421-1429

22. Olivera M, Chena J, Wonga E, Van Dyka J, Perera F (2007) A treatment planning study comparing whole breast radiation therapy against conformal, IMRT and tomotherapy for accelerated partial breast irradiation. Radiotherapy and Oncology; 82(3):317-323

23. Rybkin, AV, Thoeni, RF (2007) Current concepts in imaging of appendicitis. Radiol Clin North Am 45:411-422 
24. Macari M, Balthazar EJ (2003) The acute right lower quadrant: CT evaluation. Radiol Clin North Am 41:1117-1136.

25. O’Malley ME, Halpern E, Mueller PR, Gazelle GS (2000) Helical CT protocols for the abdomen and pelvis: a survey. AJR Am J Roentgenol 175:109-113.

26. Seo H, Lee KH, Kim HJ, Kim K, Kang SB, Kim SY, Kim YH (2009) Diagnosis of acute appendicitis with sliding slab ray-sum interpretation of low-dose unenhanced CT and standard-dose IV contrast-enhanced CT scans. AJR Am J Roentgenol 193:96-105.

27. Keyzer C, Cullus P, Tack D, Maertelaer V, Bohy P, Gevenois PA (2009) MDCT for suspected acute appendicitis in adults: impact of oral and IV contrast media at standard-dose and simulated low-dose techniques. AJR Am J Roentgenol 193:1272128.

28. Peck J, Peck A, Peck J (2000) The clinical role of noncontrast helical computed tomography in the diagnosis of acute appendicitis. Am J Surg 180:133-136.

29. Tamburrini S, Brunetti A, Brown M, Sirlin C, Casola G (2007) Acute appendicitis: diagnostic value of nonenhanced $\mathrm{CT}$ with selective use of contrast in routine clinical settings. Eur Radiol 17:2055-2061.

30. Hoecker CC, Billman GF (2005) The utility of unenhanced computed tomography in appendicitis in children. J Emerg Med 28:415-421.

31. Ege G, Akman H, Sahin A, Bugra D, Kuzucu K (2002) Diagnostic value of unenhanced helical $\mathrm{CT}$ in adult patients with suspected acute appendicitis. $\mathrm{Br} \mathrm{J}$ Radiol 75:721-725.

32. Cakirer S, Basak M, Colakoglu B, Bankaoglu M (2002) Diagnosis of acute appendicitis with unenhanced helical CT: a study of 130 patients. Emerg Radiol 9:155-161.

33. Lane MJ, Liu DM, Huynh MD, Jeffrey RB, Mindelzun RE (1999) Suspected acute appendicitis: nonenhanced helical CT in 300 consecutive patients. Radiology 213:341-346.

34. Lane MJ, Katz DS, Ross BA, Clautice-Engle TL, Mindelzun RE, Jeffrey RB (1997) Unenhanced Helical CT for Suspected Acute Appendicitis. AJR Am J Roentgenol 168:405-409.

35. Malone AJ, Wolf CR, Malmed AS, Melliere BF (1993) Diagnosis of acute appendicitis: value of unenhanced CT. AJR Am J Roentgenol 160:763-766. 
36. Anderson BA, Salem L, Flum DR (2005) A systematic review of whether oral contrast is necessary for the computed tomography diagnosis of appendicitis in adults. Am J Surg 190:474-478.

37. Benjaminov O, Atri M, Hamilton P, Rappaport D (2002) Frequency of visualization and thickness of normal appendix at nonenhanced helical CT. Radiology 225:400406.

38. Hlibczuk V, Dattaro JA, Jin Z, Falzon L, Brown MD (2010). Diagnostic accuracy of noncontract computed tomography for appendicitis in adults: a systematic review. Ann Emerg Med 55:51-59.

39. Benjaminov O, Atri M, Hamilton P, Rappaport D (2002). Frequency of visualization and thickness of normal appendix at nonenhanced helical CT. Radiology 225:400406.

40. Jan YT, Yang FS, Huang JK (2005). Visualization rate and pattern of normal appendix on multidetector computed tomography by using multiplanar reformation display. J Comput Assist Tomogr 29:446-451.

41. Grayson DE, Wettlaufer JR, Dalrymple NC, Keesling CA (2001). Appendiceal CT in pediatric patients: relationship of visualization to amount of peritoneal fat. AJR 176:497-500.

42. Karabulut N, Boyaci N, Yagci B, Herek D, Kiroglu Y (2007). Computed tomography evaluation of the normal appendix: comparison of low-dose and standard-dose unenhanced helical computed tomography. J Comput Assist Tomogr 31:732-740. 


\section{CHAPTER V}

\section{Concluding Remarks}

\subsection{Summary and thesis conclusions}

HDR lung brachytherapy is a powerful technique for palliative lung cancer treatment. A typical lung brachytherapy treatment plan is designed using orthogonal $x$-ray images. The tumour and organs at risk are not visible on x-ray images limiting the ability to optimize the treatment plan. Although planning in 3D using CT images allows for better optimized treatment plans, it is highly impractical to move patients to the CT simulator prior to each treatment. While prior diagnostic or treatment CT images are normally available, they cannot be used for treatment planning because of the difference in patient positioning. To benefit from available CT images, they have to be registered with orthogonal x-ray images. The registration of both data is required to enable treatment planning in 3D with catheters, tumor, and organs at risk in one view.

In chapter II, a 2D/3D registration algorithm was developed for registering orthogonal $\mathrm{x}$ ray images with the diagnostic CT volume for lung brachytherapy. The algorithm utilizes a rigid registration model and is based on the pixel/voxel intensity matching approach allowing for non-invasive and fully automated registration. There are a number of challenges specific to lung brachytherapy that complicate $2 \mathrm{D}$ to $3 \mathrm{D}$ registration. First, the orthogonality of an image pair acquired using a c-arm system is not guaranteed since the angles are set manually which may lead to a non-negligible random angular error. Second, respiratory motion between x-ray image acquisitions may further decrease the fidelity of the orthogonal data set. Finally, diagnostic CT data normally does not contain objects such as the ECG leads, wires, and treatment catheters present during the 
orthogonal data acquisition. All of the above factors reduce correlation between 2D and 3D data, challenging the robustness and accuracy of the registration. A similarity metric that is resistant to these unique characteristics was developed. The proposed similarity metric combines three components: NMI, image gradient, and intensity difference metrics. The best-neighbour search smoothed by parabolic interpolation was used for algorithm optimization. The optimization algorithm was modified by including a stochastic component to improve the algorithm's robustness and reduce dependence on starting parameters.

Numerical evaluation of the algorithm in which X-ray images were simulated from the CT volume of the phantom demonstrated robust and accurate registration for various degrees of artificially introduced initial displacements. The average registration error was 0.54 $\mathrm{mm}$ and $0.57 \mathrm{~mm}$ for the simple body phantom and the anthropomorphic phantom respectively. The influence of geometric errors during $\mathrm{x}$-ray image acquisition on registration accuracy was evaluated numerically. It was confirmed that registration error increases with c-arm angular deviation. The algorithm robustness was not compromised for angular deviations under $3.5^{\circ}$. The evaluation of actual phantom x-ray images indicated the robust registration of both phantoms in all investigated positions. The mean registration error was $2.2 \mathrm{~mm}$ and $1.9 \mathrm{~mm}$ for the simple body and anthropomorphic phantom respectively. The registration error was just over a half slice thickness and comparable to the mean digitization error of $1.6 \mathrm{~mm}$, which is clinically acceptable. The proposed registration algorithm also showed robustness to foreign bodies such as catheters and ECG-leads with wires.

In chapter III, the proposed registration algorithm was evaluated in a clinical setting. The evaluation was performed using data from four esophageal and two lung cancer brachytherapy treatments. The algorithm converged to a reasonable solution for all six patient datasets. The mean and maximum registration error was $3.2 \mathrm{~mm}$ and $6.1 \mathrm{~mm}$, respectively. The mean registration error of $3.2 \mathrm{~mm}$ is commensurate with the intra- and inter-observer uncertainties in the catheter digitization and with the CT slice thickness. 
The precision of the algorithm was analysed by repeatedly varying the starting parameters. The standard deviation of registration error was found to be $0.8 \mathrm{~mm}$. The algorithm was the least accurate in the axial direction, most likely due to the lower resolution of the $\mathrm{CT}$ data in that direction and due to craniocaudal motion of the diaphragm due to breathing.

In chapter IV, the algorithm for automatic removal of external objects from CT images was proposed. The objects such as the treatment table, clothing, ECG leads, wires and fiducial markers may negatively affect the quality of generated DRRs and consequently the quality of 2D/3D registration. The algorithm successfully removed the objects from ten thoracic CT data sets without affecting the pixels belonging to the patients. During the evaluation of the proposed $2 \mathrm{D} / 3 \mathrm{D}$ registration algorithm it was discovered that the algorithm was robust enough to be insensitive to the presence of external objects. Thus, CT data processing was not necessary, and original CT images were used for the algorithm's evaluation. The treatment table was removed from the CT data by manual selection of the cut off level below which pixel intensities were replaced with CT number of air.

The modified version of the algorithm was applied to automatic contouring of the fiducial markers in CT images used for external beam radiation therapy treatment planning for breast cancer. Both solder and plastic filament markers were successfully recognized and contoured for all slices of the three available CT data sets.

The algorithm was further modified to extract patient circumference and compute the girth of the patient. This algorithm was employed in a clinical study for diagnostic radiology, which required retrospective computation of the girth for 108 patients from single CT image. The computed data was used to determine if there is an abdominal girth size above which unenhanced CT scanning is adequate in identifying a normal appendix. The study confirmed that with unenhanced helical CT, the larger the patient abdominal girth measured in the supine position at the level of the iliac crests, the easier it was to identify the appendix. Unenhanced helical CT accurately showed the appendix in patients 
with girth sizes exceeding $105 \mathrm{~cm}$. For patients not meeting this girth-size criterion, an ultrasound or CT scan with contrast is recommended as primary imaging choice.

\subsection{Study Limitations and Future Directions}

Although the current level of accuracy provided by proposed 2D/3D registration algorithm may be acceptable considering palliative brachytherapy treatment scenario, a number of improvements still can be made to lower the registration error. Future improvements of the algorithm should focus on the two major sources of error: digitization error and patient motion due to breathing. Automated marker localization in both the $2 \mathrm{D}$ and $3 \mathrm{D}$ data sets should reduce the inter and intra-operator digitization variation. Accounting for errors due to respiratory motion is more problematic.

It is recognized that the general solution of the thorax registration problem can only be achieved using non-rigid methods. However, for routinely collected data, the patient anatomy deforms between orthogonal x-ray image acquisitions and also the CT data collection. This significantly reduces the correlation between the $2 \mathrm{D}$ images and the 3D volume to be registered. Furthermore, the low soft-tissue contrast in the 2D images may make deformable registration impossible, while extrapolating bony anatomy deformations to the rest of the thorax may not be sufficiently accurate. The shortage of information in the available data makes it impossible to explicitly identify additional degrees of freedom necessary in non-rigid registration without additional human interaction. Thus, a rigid transformation model is employed, benefiting from the improved robustness.

It was shown in this thesis that axial error is the biggest contribution to the algorithm registration error and that it is closely correlated with the magnitude of patient respiratory motion between acquisitions of $\mathrm{x}$-ray images. This error may be considerably reduced by synchronizing respiratory phase between c-arm images, which can be achieved with gating technique. In order to account for patient motion between x-ray and CT data 
collection, these data may require synchronizing within the respiratory cycle using a 4D CT technique. 


\section{References}

Aouadi S., Sarry L., "Accurate and precise 2D-3D registration based on X-ray intensity," Comput. Vis. Image Und. 110 134-51(2008).

Battista J. J., Rider W. D., Van Dyk J.. "Computed tomography for radiotherapy planning," International Journal of Radiation Oncology Biology Physics, 6(1) 99107 (1980).

Birkfellner W., Figl M., Kettenbach J., "Rigid 2D/3D slice-to-volume registration and its application on fluoroscopic CT images,” Med. Phys. 34246 (2007).

Celebioglu B., Gurkan O. U., Erdogan S., Savas I., Köse K., Kurtman C., Gonullu U., "High dose rate endobronchial brachytherapy effectively palliates symptoms due to inoperable lung cancer," Jpn. J. Clin. Oncol. 32(11) 443-8 (2002).

Chen X., Varley M. R., Shark L. K., Shentall G. S., Kirby M. C., “A computationally efficient method for automatic registration of orthogonal X-ray images with volumetric CT," Phys. Med. Biol. 53 967-83(2008).

Cordón O., Sergio D., Jose S., "A fast and accurate approach for 3D image registration using the scatter search evolutionary algorithm," Pattern Recognition Letters 27(11) 1191-1200 (2006).

Dagnault A., Ébacher A., Vigneault É., and Boucher S., "Retrospective study of 81 patients treated with brachytherapy for endobronchial primary tumor or metastasis," Brachytherapy 9(3) 243-7 (2010).

Dougherty E. R., Lotufo, R. A., Hands-on morphological image processing. Bellingham: Spie (2003).

Fang Z., Junpeng Z., Yulei M., Medical Image Processing Based on Mathematical Morphology, Atlantis Press (2012).

Faugeras O., Three dimensional computer vision: A geometric viewpoint. the MIT Press. (1993).

Feldmar J., Ayache N., Betting F., "3D-2D projective registration of free-form curves and surfaces," Comput. Vis. Image Und. 65(3) 403-424 (1997). 
Gueziec A., Kazanzides P., Williamson B., Taylor R. H., “Anatomy-based registration of CT-scan and intraoperative X-ray images for guiding a surgical robot," IEEE Trans. Med. Imaging 17 715-28 (1998).

Hamadeh A., Lavallée S., Cinquin P., "Automated 3-dimensional computer tomographic and fluoroscopic image registration,” Comput. Aided Surg. 3 1453-62 (1998).

Hajnal, J. V., and Hill, D. L. (Eds.). Medical image registration. CRC press. (2010).

Haralick R. M., Sternberg S. R., Zhuang X., "Image analysis using mathematical morphology," Pattern Analysis and Machine Intelligence, IEEE Transactions on; 4 532-550 (1987).

Harms W., Schraube P., Becker H., Latz D., Herth F., Fritz P., Wannenmacher M., "Effect and toxicity of endoluminal high-dose-rate (HDR) brachytherapy in centrally located tumors of the upper respiratory tract," Strahlenther Onkol. 176(2) 60-6 (2000).

Jaramaz B., Eckman K., "2D/3D registration for measurement of implant alignment after total hip replacement," MICCAI, LNCS 4191 653-61 (2006).

Khan F. M., Gibbons J., Mihailidis D. and Alkhatib H., "Khan's Lectures: handbook of the Physics of Radiation Therapy," LWW (2009).

Kim R. Y., Shen S., Duan J., "Image-based three-dimensional treatment planning of intracavitary brachytherapy for cancer of the cervix: dose-volume histograms of the bladder, rectum, sigmoid colon, and small bowel," Brachytherapy. 6(3) 187-94 (2007).

Kraats V. D., Penney G. P., Tomazevic D., Walsum T. V. and Niessen W. J., "Standardized Evaluation Methodology for 2D/3D Registration," IEEE Trans. Med. Imaging 24 1177-89 (2005).

Legg P. A., Rosin P. L., Marshall D., Morgan J. E., "Improving accuracy and efficiency of registration by mutual information using Sturges' histogram rule," In: Proc. Med. Image Understand. Anal. 26-30 (2007).

Livyatan H., Yaniv Z., Joskowicz L., "Gradient-based 2-D/3-D rigid registration of fluoroscopic X-ray to CT,” IEEE Trans. Med. Imag. 22(11) 1395-1406 (2003).

Lyczek J., Kazalski D., Kowalik L., and Sawicki M., "Comparison of the GTV coverage by PTV and isodose of $90 \%$ in 2D and 3D planning during endobronchial 
brachytherapy in the palliative treatment of patients with advanced lung cancer. Pilot study," J Contemp Brachytherapy, 4(2) 113-115 (2012).

Maes F., Collignon A., Vandermeulen D., Marchal G., and Suetens P., "Multimodality image registration by maximization of mutual information," IEEE Trans. Med. Imag. 16(2) 187-98 (1997).

Markelij P., Tomaževič D., Pernuš F., Likar B., "Optimizing bone extraction in MR Images for 3D/2D gradient based registration of MR and X-ray images," Med. Imag. 6512651224 (2007).

Markelij P., Tomaževič D., Likar B., Pernuš F., "A review of 3D/2D registration methods for image-guided interventions,” Med. Imag. Anal. 16(3) 642-661 (2012).

Melhus C. S., and Rivard M. J., "Approaches to calculating AAPM TG-43 brachytherapy dosimetry parameters for Cs, I, Ir, Pd, and Yb sources." Medical physics 331729 (2006).

Meyer C. R., Boes J. L., Kim B., Bland P. H., Zasadny K. R., Kison P. V., Koral K., Frey K. A., and Wahl R. L., "Demonstration of accuracy and clinical versatility of mutual information for automatic multimodality image fusion using affine and thinplate spline warped geometric deformations," Med. Image Anal. 1(3) 195-206 (1997).

Nam H., Renaut R. A, Chen K., Guo H., Farin G. E., "Improved inter-modality image registration using normalized mutual information with coarse-binned histograms" Commun. Numer. Meth. Eng. 25(6) 583-595 (2009).

Onal C., Arslan G., Topkan E., Pehlivan B., Yavuz M., Oymak E. and Yavuz A., "Comparison of conventional and CT-based planning for intracavitary brachytherapy for cervical cancer: target volume coverage and organs at risk doses,” J. Exp. Clin. Cancer Res. 28:95 (2009).

Penney G. P., Weese J., Little J. A., Desmedt P., Hill D. L., Hawkes D. J., “A comparison of similarity measures for use in 2-D-3-D medical image registration," IEEE Trans. Med. Imaging 17 586-95 (1998).

Penney G. P., Edwards P. J., Hipwell J. H., Slomczykowski M., Revie I., Hawkes D. J., "Postoperative calculation of acetabular cup position using 2D-3D registration," IEEE Trans. Biomed. Eng. 54(7) 1342-48 (2007). 
Pérez-Calatayud J., Granero D., Casal E., Ballester F., and Puchades V., "Monte Carlo and experimental derivation of TG43 dosimetric parameters for CSM-type Cs-137 sources." Medical Physics 3228 (2005).

Pluim J. P. W., Maintz J. B. A., Viergever M. A., "Image Registration by Maximization of Combined Mutual Information and Gradient Information," MICCAI 1935103 129 (2000).

Pluim J. P. W., Maintz J. B. A., Viergever M. A., "Mutual-information-based registration of medical images: a survey," IEEE Trans. Med. Imag. 22(8), 986-1004 (2003).

Russakoff D. B., "Intensity-based two-dimensional-three-dimensional medical image registration," Stanford University ISBN:0-496-04390-0 (2004).

Sherouse G. W., Novins K., Chaney E. L., "Computation of digitally reconstructed radiographs for use in radiotherapy treatment design," International Journal of Radiation Oncology Biology Physics 18(3) 651-658 (1990).

Studholme C., Hill D. L. G., and Hawkes D. J. "Automated three-dimensional registration of magnetic resonance and positron emission tomography brain images by multiresolution optimization of voxel similarity measures," Med. Phys. 24(1) 25-35 (1997).

Studholme C., Hill D. L. G., and Hawkes D. J., “An overlap invariant entropy measure of 3D medical image alignment," Pattern Recognit. 32(1) 71-86 (1999).

Tomaževič D., Likar B., Slivnik T., Pernu`s F., "3-D/2-D registration of CT and MR to X-ray images,” IEEE Trans. Med. Imaging 22 1101-9 (2003).

Wang R., Computer Image Processing and Analysis (E161), Lecture Notes, Faculty of Engineering, Harvey Mudd College, Claremont (2012).

Wein W., Roeper B., Navab N., "2D/3D registration based on volume gradients," Med. Imag. 5747, 144-150 (2005)

Wells W. M., Viola P., Atsumi H., Nakajima S., and Kikinis R., "Multi-modal volume registration by maximization of mutual information," Med. Image Anal. 1(1) 35-51 (1996).

Young I. T., Gerbrands J. J., van Vilet L. J., "Fundamentals of Image Processing", Delft PH Publications (1998). 
Yu E., Lewis C., Trejos A. L., Patel R. V., Malthaner R. A., "Lung Cancer Brachytherapy: Robotics-Assisted Minimally Invasive Approach," Current Respiratory Medicine Review, 7(5) 340-353 (2011).

Zaric B., Perin B., Jovelic A., Lalic N., Secen N., Kopitovic I., Antonic M., "Clinical risk factors for early complications after high-dose-rate endobronchial brachytherapy in the palliative treatment of lung cancer," Clin. Lung Cancer 11(3) 182-6 (2010).

Zheng G., "Effective incorporating spatial information in a mutual information based 3D2D registration of a CT volume to X-ray images" Computerized Medical Imaging and Graphics 34(7) 553-62 (2010). 